\title{
Reliable Low-Cost Theoretical Procedures for Studying Addition- Fragmentation in RAFT Polymerization
}

\section{Ekaterina I. Izgorodina and Michelle L. Coote *}

Research School of Chemistry, Australian National University, Canberra, ACT 0200, Australia 
Table S1. Geometries of the RAFT-adduct Radicals $\left(\mathrm{CH}_{3} \mathrm{SC} \cdot\left(\mathrm{CH}_{3}\right) \mathrm{S}-\mathrm{R}\right)$, Thiocarbonyl Products $\left(\mathrm{CH}_{3} \mathrm{SC}\left(\mathrm{CH}_{3}\right)=\mathrm{S}\right)$ and Leaving Group Radicals $(\cdot \mathrm{R})$ of BetaScission, Used in Calculating the Beta-Scission Enthalpies in Table 1

\author{
$\mathrm{CH}_{3} \mathrm{SC} \cdot\left(\mathrm{CH}_{3}\right) \mathrm{S}-\mathrm{CH}_{3}$

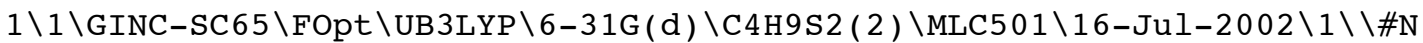 \\ UB3LYP/6-31G (D) FOPT=(Z-MATRIX, TIGHT, MAXCYC=100) FREQ MAXDISK=13107200 \\ $0 \mathrm{SCF}=(\mathrm{TIGHT}, \mathrm{MAXCYC}=100)$ GEOM=CHECK GUESS=READ $\backslash \backslash$ mescmesme $-\mathrm{C} 1 \mathrm{~b}-6 \mathrm{dub} 3 \backslash \backslash 0$ \\ , $2 \backslash \mathrm{H} \backslash \mathrm{C}, 1, \mathrm{~B} 1 \backslash \mathrm{H}, 2$, B2 , $1, \mathrm{~A} 1 \backslash \mathrm{S}, 2, \mathrm{~B} 3,1, \mathrm{~A} 2,3, \mathrm{D} 1,0 \backslash \mathrm{C}, 4, \mathrm{~B} 4,2, \mathrm{~A} 3,1, \mathrm{D} 2,0 \backslash \mathrm{S}, 5, \mathrm{~B} 5,4$ \\ , $\mathrm{A} 4,2, \mathrm{D} 3,0 \backslash \mathrm{C}, 6, \mathrm{~B} 6,5, \mathrm{~A} 5,4,-\mathrm{D} 4,0 \backslash \mathrm{H}, 7, \mathrm{~B} 7,6, \mathrm{~A} 6,5, \mathrm{D} 5,0 \backslash \mathrm{H}, 7, \mathrm{~B} 8,6, \mathrm{~A} 7,8, \mathrm{D} 6,0 \backslash \mathrm{H}$ \\ , $7, \mathrm{~B} 9,6, \mathrm{~A} 8,8, \mathrm{D} 7,0 \backslash \mathrm{C}, 5, \mathrm{~B} 10,4, \mathrm{~A} 9,2, \mathrm{D} 8,0 \backslash \mathrm{H}, 2, \mathrm{~B} 11,1, \mathrm{~A} 10,3, \mathrm{D} 9,0 \backslash \mathrm{H}, 11, \mathrm{~B} 12,5$, \\ $\mathrm{A} 11,4, \mathrm{D} 10,0 \backslash \mathrm{H}, 11, \mathrm{~B} 13,5, \mathrm{~A} 12,4, \mathrm{D} 11,0 \backslash \mathrm{H}, 11, \mathrm{~B} 14,5, \mathrm{~A} 13,4, \mathrm{D} 12,0 \backslash \backslash \mathrm{B} 1=1.092657$ \\ $93 \backslash B 2=1.09285939 \backslash B 3=1.83510395 \backslash B 4=1.77013049 \backslash B 5=1.75804982 \backslash B 6=1.832220$ \\ $08 \backslash B 7=1.09257315 \backslash B 8=1.09085787 \backslash B 9=1.09328827 \backslash B 10=1.50447696 \backslash B 11=1.0934$ \\ $6331 \backslash \mathrm{B} 12=1.09613505 \backslash \mathrm{B} 13=1.09553397 \backslash \mathrm{B} 14=1.10178689 \backslash \mathrm{A} 1=110.0297286 \backslash \mathrm{A} 2=11$ \\ $0.82443615 \backslash A 3=102.23773298 \backslash A 4=118.51568336 \backslash A 5=103.96848806 \backslash A 6=110.4950$ \\ $1693 \backslash A 7=111.21266523 \backslash A 8=105.91951214 \backslash A 9=120.43443778 \backslash A 10=109.33705389 \backslash$ \\ $\mathrm{A} 11=111.15729093 \backslash \mathrm{A} 12=110.74166016 \backslash \mathrm{A} 13=112.20742397 \backslash \mathrm{D} 1=-124.06148497 \backslash \mathrm{D} 2$ \\ $=60.56470818 \backslash \mathrm{D} 3=-144.49451711 \backslash \mathrm{D} 4=-48.977946 \backslash \mathrm{D} 5=60.52936234 \backslash \mathrm{D} 6=-122.478$ \\ $49479 \backslash D 7=118.55770463 \backslash D 8=62.40591497 \backslash D 9=119.28641302 \backslash D 10=-44.11186517 \backslash$ \\ $\mathrm{D} 11=-164.00422423 \backslash \mathrm{D} 12=75.46393503 \backslash \backslash$ Version=DEC-AXP-OSF / 1-G98RevA.11.3\ \\ $\mathrm{HF}=-954.1760596 \backslash \mathrm{S} 2=0.755169 \backslash \mathrm{S} 2-1=0 . \backslash \mathrm{S} 2 \mathrm{~A}=0.75002 \backslash \mathrm{RMSD}=5.132 \mathrm{e}-09 \backslash \mathrm{RMSF}=2$. \\ $815 e-05 \backslash \mathrm{Dipole}=0.2098476,0.5512682,-0.4657697 \backslash \mathrm{PG}=\mathrm{C} 01 \quad[\mathrm{X}(\mathrm{C} 4 \mathrm{H} 9 \mathrm{~S} 2)] \backslash \backslash @$
}

\title{
$\mathrm{CH}_{3} \mathrm{SC} \cdot\left(\mathrm{CH}_{3}\right) \mathrm{S}-\mathrm{CH}_{2} \mathrm{CH}_{3}$
}

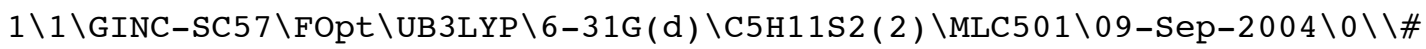

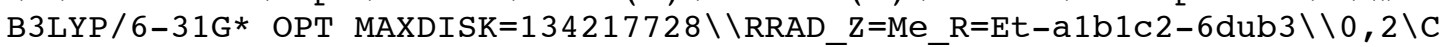
$,-0.5714512539,-0.5564439329,0.094988516 \overline{9} \backslash \mathrm{S},-\overline{0} .5650055692,-0.604787893$ $8,1.8526500644 \backslash \mathrm{S}, 0.9669930606,-0.4911502006,-0.7758965438 \backslash \mathrm{C},-1.7893355$ $415,-1.1518251078,-0.5587422291 \backslash \mathrm{C}, 0.6655047543,0.6625370943,2.33259126$ $\backslash \mathrm{C}, 0.5897960904,0.6752723494,-2.1603094186 \backslash \mathrm{H},-0.2441510744,0.273219505$ $5,-2.7446572309 \backslash \mathrm{H}, 1.4821437552,0.6213480638,-2.7933598672 \backslash \mathrm{C}, 0.31298639$ $81,2.1074427804,-1.7125615132 \backslash \mathrm{H},-2.7006113103,-0.8651638679,-0.0219773$ $509 \backslash \mathrm{H},-1.7483436946,-2.252309576,-0.5898220306 \backslash \mathrm{H},-1.8873046451,-0.8091$ $314989,-1.5953809213 \backslash \mathrm{H}, 0.6560246165,0.6890979725,3.4255499232 \backslash \mathrm{H}, 1.6639$ $613975,0.398178855,1.9816350097 \backslash \mathrm{H}, 0.3804890773,1.6415238648,1.94024960$ $01 \backslash \mathrm{H},-0.5565808062,2.1465062511,-1.0484369885 \backslash \mathrm{H}, 0.1067795092,2.7444289$ $519,-2.5819057906 \backslash \mathrm{H}, 1.1707906286,2.5254118891,-1.1757503774 \backslash \backslash$ Version=D EC-AXP-OSF / 1-G03RevB. 03 \State $=2-A \backslash H F=-993.4913813 \backslash \mathrm{S} 2=0.75518 \backslash \mathrm{S} 2-1=0 . \backslash \mathrm{S}$ $2 \mathrm{~A}=0.75002 \backslash \mathrm{RMSD}=7.019 \mathrm{e}-09 \backslash \mathrm{RMSF}=2.076 \mathrm{e}-05 \backslash \mathrm{Dipole}=-0.0510169,0.6620375,-$ $0.3780097 \backslash \mathrm{PG}=\mathrm{C} 01 \quad[\mathrm{X}(\mathrm{C} 5 \mathrm{H} 11 \mathrm{~S} 2)] \backslash \backslash \mathrm{Q}$

\section{$\mathrm{CH}_{3} \mathrm{SC} \cdot\left(\mathrm{CH}_{3}\right) \mathrm{S}-\mathrm{CH}_{2} \mathrm{CN}$}

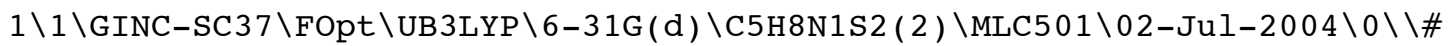
B3LYP /6-31G* OPT MAXDISK $=402653184 \backslash \backslash R R A D \_Z=C H 3 \_R=C H 2 C N-a 2 b 1 c 3-6 d u b 3 \backslash \backslash$ $0,2 \backslash S,-1.5380304454,-0.2524762319,-1.151 \overline{4} 99792 \overline{1} \backslash \mathrm{C},-0.7404267942,-0.869$ $7341876,0.2805413448 \backslash \mathrm{S}, 0.988148005,-0.9842143591,0.4748666674 \backslash \mathrm{C},-1.661$ $823209,-1.2034376129,1.423317539 \backslash \mathrm{C},-0.2024466317,0.1912427838,-2.31077$ $13878 \backslash \mathrm{C}, 1.4619280315,0.5573715331,1.4620467877 \backslash \mathrm{H}, 2.484508004,0.3682299$ $976,1.8036453886 \backslash \mathrm{H}, 0.8142951832,0.6389240576,2.3388328927 \backslash \mathrm{C}, 1.41091556$ $14,1.776935843,0.6703974501 \backslash \mathrm{H},-1.3441427705,-2.128799659,1.9167691322 \backslash$ $\mathrm{H},-2.6950123323,-1.3342674304,1.0814345642 \backslash \mathrm{H},-1.6728418189,-0.40980979$ $79,2.1870131768 \backslash \mathrm{H},-0.7091891149,0.5167715148,-3.2230978858 \backslash \mathrm{H}, 0.4008835$ $366,1.010448331,-1.9152679157 \backslash \mathrm{H}, 0.4266889358,-0.6732297701,-2.53098970$ $22 \backslash \mathrm{N}, 1.3548639536,2.726358865,0.0020857058 \backslash \backslash$ Version=DEC-AXP-OSF / 1-G03R 
evB.03\State $=2-A \backslash H F=-1046.412022 \backslash \mathrm{S} 2=0.755354 \backslash \mathrm{S} 2-1=0 . \backslash \mathrm{S} 2 \mathrm{~A}=0.750022 \backslash \mathrm{RMSD}$ $=4.672 \mathrm{e}-09 \backslash \mathrm{RMSF}=7.274 \mathrm{e}-06 \backslash \mathrm{Dipole}=-0.0365961,-0.7579938,0.5856023 \backslash \mathrm{PG}=\mathrm{C} 0$ $1[\mathrm{X}(\mathrm{C} 5 \mathrm{H} 8 \mathrm{~N} 1 \mathrm{~S} 2)] \backslash \backslash \mathrm{Q}$

\section{$\mathrm{CH}_{3} \mathrm{SC} \cdot\left(\mathrm{CH}_{3}\right) \mathrm{S}-\mathrm{C}\left(\mathrm{CH}_{3}\right)_{2} \mathrm{CN}$}

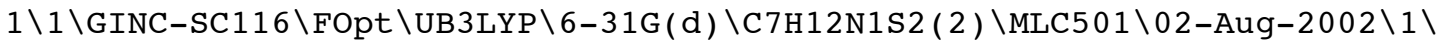
$\backslash \# \mathrm{~N}$ UB3LYP/6-31G(D) OPT $=(Z-M A T R I X, M A X C Y C=100) \quad \mathrm{SCF}=(\mathrm{MAXCYC}=100, \mathrm{QC}, \mathrm{TIGHT}$ ) FREQ MAXDISK=131072000\\RAFT_RAD( $\mathrm{Z}=\mathrm{Me} \mathrm{R}=\mathrm{Me} 2 \mathrm{CCN}) \mathrm{c} 1 \mathrm{~b} / / \mathrm{B} 3 \backslash \backslash 0,2 \backslash \mathrm{C} \backslash \mathrm{C}, 1, \mathrm{~B}$ $1 \backslash \mathrm{S}, 1, \mathrm{~B} 2,2, \mathrm{~A} 1 \backslash \mathrm{S}, 1, \mathrm{~B} 3,2, \mathrm{~A} 2,3, \mathrm{D} 1,0 \backslash \mathrm{C}, 3, \mathrm{~B} 4,1, \mathrm{~A} 3,2, \mathrm{D} 2,0 \backslash \mathrm{C}, 4, \mathrm{~B} 5,1, \mathrm{~A} 4,2, \mathrm{D} 3,0$ $\backslash \mathrm{C}, 6, \mathrm{~B} 6,4, \mathrm{~A} 5,1, \mathrm{D} 4,0 \backslash \mathrm{C}, 6, \mathrm{~B} 7,4, \mathrm{~A} 6,1, \mathrm{D} 5,0 \backslash \mathrm{C}, 6, \mathrm{~B} 8,4, \mathrm{~A} 7,1, \mathrm{D} 6,0 \backslash \mathrm{H}, 5, \mathrm{~B} 9,3, \mathrm{~A} 8$, 1 , D7 , 0\H , $5, \mathrm{~B} 10,3, \mathrm{~A} 9,10, \mathrm{D} 8,0 \backslash \mathrm{H}, 5, \mathrm{~B} 11,3, \mathrm{~A} 10,10, \mathrm{D} 9,0 \backslash \mathrm{H}, 2, \mathrm{~B} 12,1, \mathrm{~A} 11,3, \mathrm{D} 10$, $0 \backslash \mathrm{H}, 2, \mathrm{~B} 13,1, \mathrm{~A} 12,13, \mathrm{D} 11,0 \backslash \mathrm{H}, 2, \mathrm{~B} 14,1, \mathrm{~A} 13,13, \mathrm{D} 12,0 \backslash \mathrm{H}, 7, \mathrm{~B} 15,6, \mathrm{~A} 14,4, \mathrm{D} 13,0 \backslash$ $\mathrm{H}, 7, \mathrm{~B} 16,6, \mathrm{~A} 15,16, \mathrm{D} 14,0 \backslash \mathrm{H}, 7, \mathrm{~B} 17,6, \mathrm{~A} 16,16, \mathrm{D} 15,0 \backslash \mathrm{H}, 8, \mathrm{~B} 18,6, \mathrm{~A} 17,4, \mathrm{D} 16,0 \backslash \mathrm{H}$, $8, \mathrm{~B} 19,6, \mathrm{~A} 18,19, \mathrm{D} 17,0 \backslash \mathrm{H}, 8, \mathrm{~B} 20,6, \mathrm{~A} 19,19, \mathrm{D} 18,0 \backslash \mathrm{X}, 9,1 ., 6,90 ., 4, \mathrm{D} 19,0 \backslash \mathrm{N}, 9, \mathrm{~B}$ $21,22, \mathrm{~A} 20,6,180$. $0 \backslash \backslash \mathrm{D} 2=16.38295078 \backslash \mathrm{D} 3=77.64088756 \backslash \mathrm{D} 4=60.37483458 \backslash \mathrm{D} 5=-1$ $78.7383868 \backslash \mathrm{D} 6=-61.16988989 \backslash \mathrm{B} 1=1.50208945 \backslash \mathrm{B} 2=1.75657198 \backslash \mathrm{B} 3=1.74732399 \backslash \mathrm{B}$ $4=1.83034897 \backslash \mathrm{B} 5=1.91282884 \backslash \mathrm{B} 6=1.53539307 \backslash \mathrm{B} 7=1.5395273 \backslash \mathrm{B} 8=1.46607825 \backslash \mathrm{B} 9$ $=1.09303629 \backslash \mathrm{B} 10=1.092321 \backslash \mathrm{B} 11=1.09293827 \backslash \mathrm{B} 12=1.10007771 \backslash \mathrm{B} 13=1.09890754 \backslash$ $\mathrm{B} 14=1.09212294 \backslash \mathrm{B} 15=1.09292583 \backslash \mathrm{B} 16=1.09396533 \backslash \mathrm{B} 17=1.09672447 \backslash \mathrm{B} 18=1.0945$ $1538 \backslash \mathrm{B} 19=1.09522691 \backslash \mathrm{B} 20=1.09354597 \backslash \mathrm{B} 21=1.16247912 \backslash \mathrm{A} 1=122.50686981 \backslash \mathrm{A} 2=1$ $23.17398239 \backslash A 3=104.46655439 \backslash A 4=106.49858295 \backslash A 5=110.98235599 \backslash A 6=104.639$ $96652 \backslash A 7=108.61843008 \backslash A 8=105.70641764 \backslash A 9=111.77488663 \backslash A 10=111.17328243$ $\backslash A 11=112.07996327 \backslash A 12=111.90892444 \backslash A 13=110.10196343 \backslash A 14=111.36166171 \backslash A$ $15=109.52774174 \backslash A 16=110.12678 \backslash A 17=109.62280482 \backslash A 18=109.87509857 \backslash A 19=11$ $1.407113 \backslash \mathrm{A} 20=89.82090986 \backslash \mathrm{D} 1=-177.90491864 \backslash \mathrm{D} 7=180.96073931 \backslash \mathrm{D} 8=118.42528$ $654 \backslash D 9=-118.08928553 \backslash D 10=64.15148444 \backslash D 11=-120.40351119 \backslash D 12=119.4744608$ $\backslash \mathrm{D} 13=-60.04758136 \backslash \mathrm{D} 14=120.04550823 \backslash \mathrm{D} 15=-120.27316252 \backslash \mathrm{D} 16=-61.18845374 \backslash$ $\mathrm{D} 17=-119.2157694 \backslash \mathrm{D} 18=120.55449916 \backslash \mathrm{D} 19=166.9636757 \backslash \backslash$ Version=DEC $-\mathrm{AXP}-\mathrm{OSF}$ /1-G98RevA. 11.3 $\backslash \mathrm{HF}=-1125.0409306 \backslash \mathrm{S} 2=0.755753 \backslash \mathrm{S} 2-1=0 . \backslash \mathrm{S} 2 \mathrm{~A}=0.750025 \backslash \mathrm{RMSD}$ $=0.000 e+00 \backslash \mathrm{RMSF}=9.570 \mathrm{e}-05 \backslash \mathrm{Dipole}=0.9525901,-0.1520827,-0.5410097 \backslash \mathrm{PG}=\mathrm{C} 0$ $1[\mathrm{X}(\mathrm{C} 7 \mathrm{H} 12 \mathrm{~N} 1 \mathrm{~S} 2)] \backslash \backslash \mathrm{Q}$

\section{$\mathrm{CH}_{3} \mathrm{SC} \cdot\left(\mathrm{CH}_{3}\right) \mathrm{S}-\mathrm{CH}_{2} \mathrm{COOCH}_{3}$}

$1 \backslash 1 \backslash G I N C-S C 26 \backslash F O p t \backslash U B 3 L Y P \backslash 6-31 G(d) \backslash C 6 H 1102 S 2$ ( 2) \MLC501\02-Aug-2002 $1 \backslash \backslash$ \# UB3LYP/6-31G* OPT=Z-MATRIX SCF $=(\mathrm{QC}, \mathrm{MAXCYC}=100, \mathrm{TIGHT})$ FREQ MAXDISK=13 $1072000 \backslash \backslash$ RAFT_RAD $(\mathrm{Z}=\mathrm{Me} \mathrm{R}=\mathrm{MA}) \mathrm{c} 1 \mathrm{p} / / \mathrm{B} 3 \backslash \backslash 0,2 \backslash \mathrm{S} \backslash \mathrm{C}, 1, \mathrm{~B} 1 \backslash \mathrm{C}, 2, \mathrm{~B} 2,1, \mathrm{~A} 1 \backslash \mathrm{S}, 2$, B3, $1, \mathrm{~A} 2,3, \mathrm{D} 1,0 \backslash \mathrm{H}, 3, \mathrm{~B} 4,2, \mathrm{~A} 3,1, \mathrm{D} 2,0 \backslash \mathrm{H}, 3, \mathrm{~B} 5,2, \mathrm{~A} 4,5, \mathrm{D} 3,0 \backslash \mathrm{H}, 3, \mathrm{~B} 6,2, \mathrm{~A} 5,5, \mathrm{D} 4,0 \backslash \mathrm{C}$ $, 1, \mathrm{~B} 7,2, \mathrm{~A} 6,3, \mathrm{D} 5,0 \backslash \mathrm{C}, 4, \mathrm{~B} 8,2, \mathrm{~A} 7,3, \mathrm{D} 6,0 \backslash \mathrm{H}, 9, \mathrm{~B} 9,4, \mathrm{~A} 8,2, \mathrm{D} 7,0 \backslash \mathrm{H}, 9, \mathrm{~B} 10,4, \mathrm{~A} 9,2$ , $\mathrm{D} 8,0 \backslash \mathrm{C}, 9$, $\mathrm{B} 11,4, \mathrm{~A} 10,2$, $\mathrm{D} 9,0 \backslash 0,12, \mathrm{~B} 12,9, \mathrm{~A} 11,4, \mathrm{D} 10,0 \backslash 0,12, \mathrm{~B} 13,9, \mathrm{~A} 12,13$, D1 $1,0 \backslash \mathrm{C}, 14, \mathrm{~B} 14,12, \mathrm{~A} 13,13, \mathrm{D} 12,0 \backslash \mathrm{H}, 8, \mathrm{~B} 15,1, \mathrm{~A} 14,2, \mathrm{D} 13,0 \backslash \mathrm{H}, 8, \mathrm{~B} 16,1, \mathrm{~A} 15,16, \mathrm{D} 1$ $4,0 \backslash \mathrm{H}, 8, \mathrm{~B} 17,1, \mathrm{~A} 16,16, \mathrm{D} 15,0 \backslash \mathrm{H}, 15, \mathrm{~B} 18,14, \mathrm{~A} 17,12, \mathrm{D} 16,0 \backslash \mathrm{H}, 15, \mathrm{~B} 19,14, \mathrm{~A} 18,19$ $, \mathrm{D} 17,0 \backslash \mathrm{H}, 15, \mathrm{~B} 20,14, \mathrm{~A} 19,19, \mathrm{D} 18,0 \backslash \backslash \mathrm{D} 5=-47.66210571 \backslash \mathrm{D} 6=124.2034009 \backslash \mathrm{D} 7=51$. $45345692 \backslash D 8=169.97852761 \backslash D 9=-69.03688301 \backslash D 10=79.59632628 \backslash D 11=-178.3012$ $6539 \backslash D 12=-1.10736436 \backslash D 13=-176.1569452 \backslash D 14=-118.70056952 \backslash D 15=118.684277$ $86 \backslash \mathrm{D} 16=59.84254391 \backslash \mathrm{D} 17=-120.7098879 \backslash \mathrm{D} 18=119.71257159 \backslash \mathrm{B} 1=1.75470101 \backslash \mathrm{B} 2=$ $1.50144142 \backslash \mathrm{B} 3=1.7540458 \backslash \mathrm{B} 4=1.09580114 \backslash \mathrm{B} 5=1.101524 \backslash \mathrm{B} 6=1.09607085 \backslash \mathrm{B} 7=1.8$ $3909477 \backslash B 8=1.85893744 \backslash B 9=1.09152394 \backslash B 10=1.09162639 \backslash B 11=1.50894962 \backslash B 12=$ $1.21538556 \backslash \mathrm{B} 13=1.34868612 \backslash \mathrm{B} 14=1.43745151 \backslash \mathrm{B} 15=1.09346144 \backslash \mathrm{B} 16=1.09284121$ $\backslash \mathrm{B} 17=1.09195971 \backslash \mathrm{B} 18=1.0931971 \backslash \mathrm{B} 19=1.09330407 \backslash \mathrm{B} 20=1.09019523 \backslash \mathrm{A} 1=122.104$ $41177 \backslash \mathrm{A} 2=118.7219121 \backslash \mathrm{A} 3=111.19302428 \backslash \mathrm{A} 4=111.34102025 \backslash \mathrm{A} 5=111.01263129 \backslash \mathrm{A}$ $6=102.5326864 \backslash A 7=103.45710004 \backslash A 8=108.16559651 \backslash A 9=105.69370446 \backslash A 10=110$. $77782324 \backslash A 11=124.84467967 \backslash A 12=111.49500109 \backslash A 13=115.39415873 \backslash A 14=106.25$ $208603 \backslash A 15=111.51685978 \backslash A 16=109.15493098 \backslash A 17=110.56758888 \backslash A 18=110.4339$ $1008 \backslash A 19=105.51868249 \backslash D 1=179.45105199 \backslash D 2=-31.67767788 \backslash D 3=120.39887003 \backslash$ $\mathrm{D} 4=-120.58711712 \backslash \backslash$ Version=DEC-AXP-OSF / 1-G98RevA. $11.3 \backslash \mathrm{HF}=-1182.0481894 \backslash$ $\mathrm{S} 2=0.756239 \backslash \mathrm{S} 2-1=0 . \backslash \mathrm{S} 2 \mathrm{~A}=0.750029 \backslash \mathrm{RMSD}=0.000 \mathrm{e}+00 \backslash \mathrm{RMSF}=9.546 \mathrm{e}-05 \backslash \mathrm{Dipole}=$ 


\section{$0.1388335,0.8127896,0.3170917 \backslash \mathrm{PG}=\mathrm{C} 01 \quad[\mathrm{X}(\mathrm{C} 6 \mathrm{H} 11 \mathrm{O} 2 \mathrm{~S} 2)] \backslash \backslash @$}

\section{$\mathrm{CH}_{3} \mathrm{SC} \cdot\left(\mathrm{CH}_{3}\right) \mathrm{S}-\mathrm{CH}\left(\mathrm{COOCH}_{3}\right) \mathrm{CH}_{3}$}

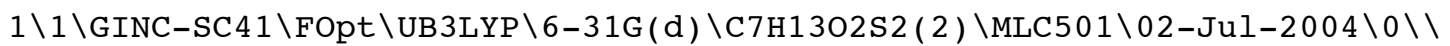

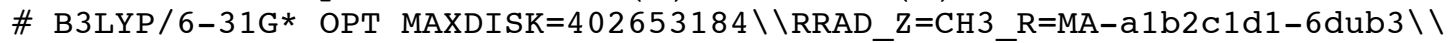
$0,2 \backslash \mathrm{S},-1.7064513367,0.4585471093,-1.5479560188 \backslash \mathrm{C},-1.2612108146,1.04526$ $09237,0.0502760557 \backslash \mathrm{S}, 0.3377633178,1.6085496057,0.4567359387 \backslash \mathrm{C},-2.30401$ $28814,0.8237776351,1.1115679166 \backslash \mathrm{C},-0.2516883218,0.7831379262,-2.601002$ $536 \backslash \mathrm{C}, 1.2006265638,0.1564804575,1.3316568904 \backslash \mathrm{H}, 0.6808602914,-0.0107128$ $881,2.2767599836 \backslash \mathrm{C}, 2.6517975984,0.5780518012,1.5496997151 \backslash \mathrm{C}, 1.08699856$ $2,-1.0778806516,0.4641796181 \backslash 0,1.7816236346,-1.3279580664,-0.499868041$ $5 \backslash 0,0.0789716746,-1.871559552,0.8866011094 \backslash \mathrm{C},-0.1738325958,-3.03429924$ $41,0.0774023692 \backslash \mathrm{H},-0.5174281462,-2.7325227938,-0.9154738363 \backslash \mathrm{H},-0.95426$ $1989,-3.5885020463,0.5992773686 \backslash \mathrm{H}, 0.7317580007,-3.6378841986,-0.019532$ $1117 \backslash \mathrm{H}, 2.701961556,1.5313433776,2.0851304809 \backslash \mathrm{H}, 3.1776135095,-0.1759524$ $768,2.1465507044 \backslash \mathrm{H}, 3.1684067131,0.6769568008,0.5917850181 \backslash \mathrm{H}, 0.61653014$ $03,0.2218835912,-2.2508230034 \backslash \mathrm{H},-0.0225612104,1.8502225602,-2.62878119$ $04 \backslash \mathrm{H},-0.5355180187,0.4441331316,-3.6010977948 \backslash \mathrm{H},-2.2929722427,1.646834$ $1297,1.8346396306 \backslash \mathrm{H},-2.1219234559,-0.1103082479,1.6644324339 \backslash \mathrm{H},-3.3102$ $879845,0.7599294787,0.6801088804 \backslash \backslash$ Version=DEC-AXP-OSF / 1-G03RevB.03 \Sta $\mathrm{te}=2-\mathrm{A} \backslash \mathrm{HF}=-1221.3644856 \backslash \mathrm{S} 2=0.755577 \backslash \mathrm{S} 2-1=0 . \backslash \mathrm{S} 2 \mathrm{~A}=0.750024 \backslash \mathrm{RMSD}=3.906 \mathrm{e}-0$ $9 \backslash \mathrm{RMSF}=8.649 \mathrm{e}-06 \backslash \mathrm{Dipole}=-0.1894324,-0.5089002,0.3515619 \backslash \mathrm{PG}=\mathrm{C} 01] \mathrm{X}(\mathrm{C} 7 \mathrm{H} 1$ $302 \mathrm{~S} 2)] \backslash \backslash$ Q

\section{$\mathrm{CH}_{3} \mathrm{SC} \cdot\left(\mathrm{CH}_{3}\right) \mathrm{S}-\mathrm{CH}_{2} \mathrm{OCOCH}$}

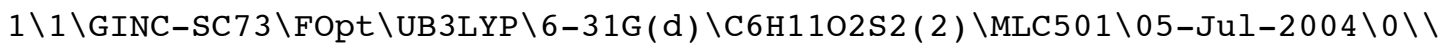

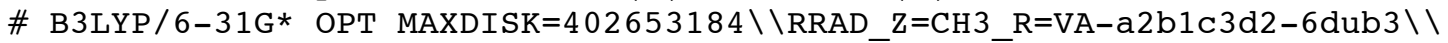
$0,2 \backslash \mathrm{S},-1.6046335589,1.301522146,0.53475091 \overline{7} 7 \backslash \mathrm{C},-\overline{1} .4065096437,-0.439314$ $5101,0.3581824035 \backslash \mathrm{S},-0.8922660445,-1.1901341949,-1.1411006036 \backslash \mathrm{C},-1.410$ $2262235,-1.2085219907,1.6498746478 \backslash \mathrm{C},-1.6293436422,1.946206834,-1.1714$ $637404 \backslash \mathrm{C}, 0.9204643955,-1.4881324727,-0.901496867 \backslash \mathrm{H}, 1.2448792839,-2.005$ $007546,-1.8074296125 \backslash \mathrm{H}, 1.1013951504,-2.0956108275,-0.0156311481 \backslash 0,1.66$ $63599954,-0.2736770225,-0.8152836802 \backslash \mathrm{C}, 2.1365151208,0.0803063968,0.416$ $6937089 \backslash 0,2.0206915597,-0.602470372,1.4092452717 \backslash \mathrm{C}, 2.7951658799,1.4353$ $600192,0.3592736658 \backslash \mathrm{H}, 2.0163081307,2.2065424717,0.3847818106 \backslash \mathrm{H}, 3.44278$ $26674,1.5604714006,1.2281279912 \backslash \mathrm{H}, 3.3624830668,1.5594511897,-0.5665777$ $672 \backslash \mathrm{H},-0.3943232028,-1.3161464413,2.0583917286 \backslash \mathrm{H},-1.8186436244,-2.2128$ $548495,1.4912150784 \backslash \mathrm{H},-2.0216995741,-0.7062569417,2.4091051623 \backslash \mathrm{H},-1.75$ $54986892,3.0281972936,-1.0776828902 \backslash \mathrm{H},-0.6929168657,1.7235331138,-1.68$ $59865348 \backslash \mathrm{H},-2.4671804492,1.5292274153,-1.7347944871 \backslash \backslash$ Version=DEC $-\mathrm{AXP}-\mathrm{O}$ $\mathrm{SF} / 1-\mathrm{G} 03 \mathrm{RevB} .03 \backslash \mathrm{State}=2-\mathrm{A} \backslash \mathrm{HF}=-1182.0520959 \backslash \mathrm{S} 2=0.755234 \backslash \mathrm{S} 2-1=0 . \backslash \mathrm{S} 2 \mathrm{~A}=0.7$ $50021 \backslash \mathrm{RMSD}=5.465 \mathrm{e}-09 \backslash \mathrm{RMSF}=4.441 \mathrm{e}-06 \backslash \mathrm{Dipole}=0.3543029,0.4721383,-0.5770$ $369 \backslash \mathrm{PG}=\mathrm{C} 01 \quad[\mathrm{X}(\mathrm{C} 6 \mathrm{H} 1102 \mathrm{~S} 2)] \backslash \backslash @$

\section{$\mathrm{CH}_{3} \mathrm{SC} \cdot\left(\mathrm{CH}_{3}\right) \mathrm{S}-\mathrm{CH}_{2} \mathrm{Ph}$}

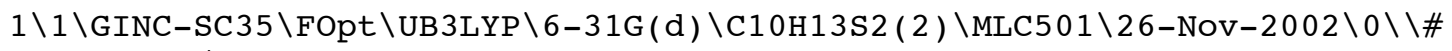
$\mathrm{N}$ UB3LYP/6-31G(D) OPT $=(\mathrm{MAXCYC}=100)$ FREQ MAXDISK=131072000 GUESS=READ G EOM=CHECK $\backslash \backslash R A F T-R A D(Z=M e ~ R=B z) \quad c 1 c y \backslash \backslash 0,2 \backslash C, 0.5508217904,1.7349275973,0$ $.082764896 \backslash \mathrm{C}, 0.56372646,1.7473599479,1.5871934348 \backslash \mathrm{S}, 2.1301757851,1.717$ $3441418,-0.6950929289 \backslash \mathrm{C}, 1.7948574291,2.2440328176,-2.4132160553 \backslash \mathrm{S},-0.9$ $429428737,1.4302432185,-0.7838339056 \backslash \mathrm{C},-0.8526164377,-0.3899166038,-1$. $2702362419 \backslash \mathrm{H}, 0.0428795461,-0.5253130123,-1.8826001106 \backslash \mathrm{H},-1.7299201211$, $-0.5085772565,-1.9145364482 \backslash \mathrm{C},-0.875490546,-1.3501477238,-0.1161572952$ $\backslash \mathrm{C},-2.0833512206,-1.7022349815,0.5041883734 \backslash \mathrm{C}, 0.3160949475,-1.90654556$ $57,0.3697099505 \backslash \mathrm{C},-2.1004443834,-2.5879588179,1.5797548248 \backslash \mathrm{C}, 0.3015747$ $185,-2.7911897776,1.4498907745 \backslash \mathrm{C},-0.9065670386,-3.1349590939,2.0577562$ $329 \backslash \mathrm{H},-3.0147042772,-1.2737279948,0.1408925506 \backslash \mathrm{H}, 1.2593017662,-1.64381$ 
$76211,-0.1034607552 \backslash \mathrm{H},-3.0460141015,-2.8536537482,2.0452370028 \backslash \mathrm{H}, 1.318$ $5665671,3.2267422653,-2.4245626833 \backslash \mathrm{H}, 1.1643996451,1.5261239453,-2.9404$ $378065 \backslash \mathrm{H},-0.9197307406,-3.8264159381,2.8960029584 \backslash \mathrm{H}, 1.2346124547,-3.21$ $48198333,1.8123905538 \backslash \mathrm{H},-0.3169489145,2.2771850403,1.9681460829 \backslash \mathrm{H}, 1.46$ $03717508,2.2435428602,1.9759037853 \backslash \mathrm{H}, 0.5291283588,0.7289034058,2.00268$ $91135 \backslash \mathrm{H}, 2.7706971684,2.3022233323,-2.9027282589 \backslash \backslash$ Version=DEC-AXP-OSF $/ 1$ -G98RevA. $11.3 \backslash \mathrm{HF}=-1185.2273621 \backslash \mathrm{S} 2=0.755783 \backslash \mathrm{S} 2-1=0 . \backslash \mathrm{S} 2 \mathrm{~A}=0.750026 \backslash \mathrm{RMSD}=6$ $.979 e-09 \backslash \mathrm{RMSF}=3.861 \mathrm{e}-06 \backslash \mathrm{Dipole}=0.0663036,-0.3509731,-0.2514008 \backslash \mathrm{PG}=\mathrm{C} 01$ $[\mathrm{X}(\mathrm{C} 10 \mathrm{H} 13 \mathrm{~S} 2)] \backslash \backslash @$

\section{$\mathrm{CH}_{3} \mathrm{SC} \cdot\left(\mathrm{CH}_{3}\right) \mathrm{S}-\mathrm{CH}(\mathrm{Ph}) \mathrm{CH}_{3}$}

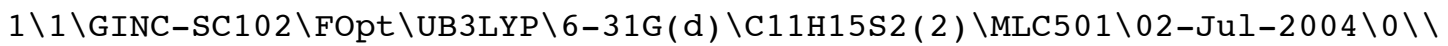

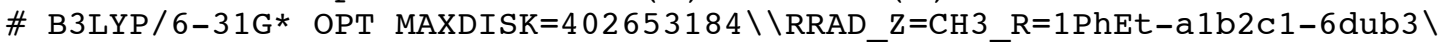
$\backslash 0,2 \backslash \mathrm{C},-1.2176028819,-1.6843968962,-0.3913 \overline{2} 91999 \backslash \mathrm{S},-1.9093817162,-1.45$ $69399145,1.210340452 \backslash \mathrm{S}, 0.492000055,-1.7258102067,-0.7274888115 \backslash \mathrm{C},-2.22$ $70532069,-1.6816464488,-1.5075943313 \backslash C,-0.4856943054,-1.455898616,2.34$ $72188923 \backslash \mathrm{C}, 0.905563721,-0.0301559236,-1.4995679164 \backslash \mathrm{H}, 0.1684905209,0.11$ $44142062,-2.2948715988 \backslash \mathrm{C}, 2.2939197209,-0.1870189968,-2.1290697854 \backslash \mathrm{C}, 0$. $7901810611,1.1014563651,-0.5086570492 \backslash C,-0.268738682,2.0138033376,-0.6$ $09791689 \backslash \mathrm{C}, 1.7234478311,1.2794405377,0.5262090162 \backslash \mathrm{H},-1.001739103,1.892$ $935148,-1.4043876911 \backslash \mathrm{H}, 2.5475627385,0.5794491486,0.6344684159 \backslash \mathrm{C},-0.393$ $5815157,3.0763941887,0.2874748765 \backslash \mathrm{C}, 1.6032366883,2.3404981666,1.421944$ $1074 \backslash \mathrm{H},-1.2233340838,3.7712310984,0.1878411758 \backslash \mathrm{H}, 2.3390809309,2.463207$ $5975,2.2126288562 \backslash \mathrm{C}, 0.5433928057,3.2446716206,1.3061911109 \backslash \mathrm{H}, 0.4508520$ $574,4.0712806966,2.0054491189 \backslash \mathrm{H}, 2.2879901725,-0.9765257367,-2.88681598$ $16 \backslash \mathrm{H}, 2.5957253405,0.752610391,-2.6054219417 \backslash \mathrm{H}, 3.0532948084,-0.44291187$ $94,-1.3825937077 \backslash \mathrm{H},-2.3272882621,-0.6892222734,-1.9757620291 \backslash \mathrm{H},-3.2232$ $911537,-1.9691594413,-1.149896426 \backslash \mathrm{H},-1.9290035856,-2.385655656,-2.2925$ $617271 \backslash \mathrm{H},-0.908950459,-1.3353182463,3.348187309 \backslash \mathrm{H}, 0.0595783422,-2.4005$ $274628,2.2923542788 \backslash \mathrm{H}, 0.1867108979,-0.62468966,2.127587509 \backslash \backslash$ Version=DE C-AXP-OSF / 1-G03RevB.03 \State $=2-\mathrm{A} \backslash \mathrm{HF}=-1224.5411627 \backslash \mathrm{S} 2=0.7558 \backslash \mathrm{S} 2-1=0 . \backslash \mathrm{S} 2$ $\mathrm{A}=0.750026 \backslash \mathrm{RMSD}=5.360 \mathrm{e}-09 \backslash \mathrm{RMSF}=2.368 \mathrm{e}-06 \backslash \mathrm{Dipole}=0.2447162,0.5788032,-0$ $.0008352 \backslash \mathrm{PG}=\mathrm{C} 01 \quad[\mathrm{X}(\mathrm{C} 11 \mathrm{H} 15 \mathrm{~S} 2)] \backslash \backslash \mathrm{Q}$

\section{$\mathrm{CH}_{3} \mathrm{SC} \cdot\left(\mathrm{CH}_{3}\right) \mathrm{S}-\mathrm{C}\left(\mathrm{CH}_{3}\right)_{2}(\mathrm{Ph})$}

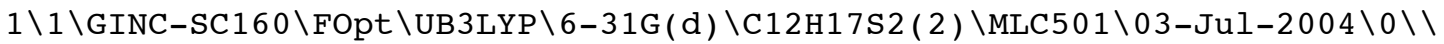

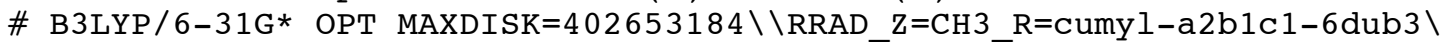
$\backslash 0,2 \backslash \mathrm{S},-0.7628524491,-1.2971839506,-2.9011 \overline{1} 65436 \backslash \mathrm{C},-0.7609963136,-1.27$ $03856948,-1.1372513449 \backslash \mathrm{S}, 0.6697574984,-1.4847300919,-0.1650731362 \backslash \mathrm{C},-2$ $.089733426,-0.9017572069,-0.5312818688 \backslash \mathrm{C}, 0.8612300309,-1.9807864649,-3$ $.368182635 \backslash \mathrm{C}, 1.2946597608,0.2719088123,0.359805165 \backslash \mathrm{C}, 1.6655293204,1.04$ $80335395,-0.9068197654 \backslash \mathrm{C}, 2.5708895557,-0.0445550805,1.1613234649 \backslash \mathrm{C}, 0.2$ $56795598,0.9683180919,1.2348574442 \backslash \mathrm{C},-0.2901611371,2.2143412637,0.8949$ $467456 \backslash \mathrm{C},-0.1672408748,0.3771723814,2.4387969246 \backslash \mathrm{H}, 0.0056315575,2.7087$ $022422,-0.0237138193 \backslash \mathrm{H}, 0.2157105452,-0.5998811096,2.7176946421 \backslash \mathrm{C},-1.22$ $09811186,2.8473646623,1.724377549 \backslash \mathrm{C},-1.0912742617,1.0062878448,3.26832$ $36995 \backslash \mathrm{H},-1.6266789317,3.8127233564,1.432601424 \backslash \mathrm{H},-1.4001300009,0.52273$ $46745,4.1915814897 \backslash \mathrm{C},-1.6252226329,2.2486104916,2.9150881084 \backslash \mathrm{H},-2.3485$ $529916,2.7396299541,3.5604953541 \backslash \mathrm{H}, 3.2989578374,-0.5744220121,0.537825$ $6373 \backslash \mathrm{H}, 3.0267394686,0.8924412995,1.5034040502 \backslash \mathrm{H}, 2.366490215,-0.6582555$ $113,2.043009512 \backslash \mathrm{H}, 2.4295062665,0.5003556057,-1.4662402173 \backslash \mathrm{H}, 2.08025262$ $07,2.0316548996,-0.650744128 \backslash \mathrm{H}, 0.8053237197,1.1988773794,-1.5658151427$ $\backslash \mathrm{H},-2.2052476002,-1.3869544428,0.4433642738 \backslash \mathrm{H},-2.9208377553,-1.2140573$ $754,-1.1756217891 \backslash \mathrm{H},-2.1854207888,0.1808771702,-0.3649881256 \backslash \mathrm{H}, 0.85454$ $18182,-2.0395329587,-4.4599884474 \backslash \mathrm{H}, 1.6746622,-1.3318599913,-3.0394893$ $937 \backslash \mathrm{H}, 0.9976040247,-2.9797243428,-2.9482413655 \backslash \backslash$ Version=DEC-AXP-OSF / 1G03RevB.03 $\backslash$ State $=2-A \backslash H F=-1263.8510387 \backslash S 2=0.756265 \backslash S 2-1=0 . \backslash S 2 A=0.750029$ 
$\backslash \mathrm{RMSD}=6.961 \mathrm{e}-09 \backslash \mathrm{RMSF}=1.837 \mathrm{e}-06 \backslash \mathrm{Dipole}=0.351457,0.422136,0.0442288 \backslash \mathrm{PG}=\mathrm{C}$ $01[\mathrm{X}(\mathrm{C} 12 \mathrm{H} 17 \mathrm{~S} 2)] \backslash \backslash @$

\section{$\mathrm{S}=\mathrm{C}\left(\mathrm{CH}_{3}\right) \mathrm{SCH}_{3}$}

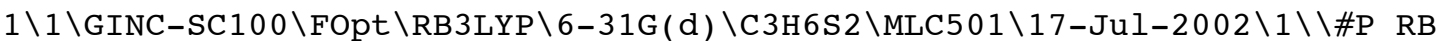
$3 \mathrm{LYP} / 6-31 \mathrm{G} *$ FOPT $=($ MAXCYC $=150, \mathrm{Z}-\mathrm{MATRIX})$ MAXDISK $=131072000 \mathrm{FREQ} \quad \mathrm{SCF}=\mathrm{TIGH}$ T GEOM=CHECK GUESS=READ $\backslash \backslash \mathrm{S}=\mathrm{C}(\mathrm{Me})-\mathrm{S}-\mathrm{Me} \mathrm{C} 1 \backslash \backslash 0,1 \backslash \mathrm{S} \backslash \mathrm{C}, 1, \mathrm{~b} 1 \backslash \mathrm{C}, 2, \mathrm{~b} 2,1, \mathrm{a} 1 \backslash \mathrm{S}, 2$ , b3 , 1 , a 2 , 3 , dh $3,0 \backslash \mathrm{C}, 4, \mathrm{~b} 4,2, \mathrm{a} 3,1, \mathrm{dh} 4,0 \backslash \mathrm{H}, 5, \mathrm{~b} 5,4, \mathrm{a} 4,2, \mathrm{dh} 5,0 \backslash \mathrm{H}, 5, \mathrm{~b} 6,4, \mathrm{a} 5,2$ , dh $1,0 \backslash \mathrm{H}, 5, \mathrm{~b} 6 \mathrm{a}, 4, \mathrm{a} 5 \mathrm{a}, 2,-\mathrm{dh} 1 \mathrm{a}, 0 \backslash \mathrm{H}, 3, \mathrm{~b} 7,2, \mathrm{a} 6,1, \mathrm{dh} 6,0 \backslash \mathrm{H}, 3, \mathrm{~b} 8,2, \mathrm{a} 7,1, \mathrm{dh} 2,0$ $\backslash \mathrm{H}, 3, \mathrm{~b} 8 \mathrm{a}, 2, \mathrm{a} 7 \mathrm{a}, 1,-\mathrm{dh} 2 \mathrm{a}, 0 \backslash \backslash \mathrm{b} 1=1.64599636 \backslash \mathrm{b} 2=1.5148461 \backslash \mathrm{b} 3=1.75511783 \backslash \mathrm{b} 4=$ $1.81794969 \backslash \mathrm{b} 5=1.09307671 \backslash \mathrm{b} 6=1.0919738 \backslash \mathrm{b} 6 \mathrm{a}=1.09197036 \backslash \mathrm{b} 7=1.09324611 \backslash \mathrm{b} 8=$ $1.09537804 \backslash \mathrm{b} 8 \mathrm{a}=1.0962657 \backslash \mathrm{a} 1=122.84648556 \backslash \mathrm{a} 2=125.75342086 \backslash \mathrm{a} 3=103.776055$ $3 \backslash a 4=106.19073532 \backslash a 5=110.33849971 \backslash a 5 a=110.27255399 \backslash a 6=109.69980988 \backslash a 7=$ $112.81878244 \backslash a 7 a=109.03573022 \backslash d h 1=60.70823437 \backslash d h 1 a=60.13720525 \backslash d h 2=167$ $.56947484 \backslash \mathrm{dh} 2 \mathrm{a}=72.03339216 \backslash \mathrm{dh} 3=181.23401229 \backslash \mathrm{dh} 4=0.90237034 \backslash \mathrm{dh} 5=180.305$ $77272 \backslash \mathrm{dh} 6=45.4338183 \backslash \backslash$ Version $=\mathrm{DEC}-\mathrm{AXP}-\mathrm{OSF} / 1-\mathrm{G} 98 \mathrm{RevA} .11 .3 \backslash \mathrm{HF}=-914.30737$ $69 \backslash \mathrm{RMSD}=3.465 \mathrm{e}-10 \backslash \mathrm{RMSF}=1.676 \mathrm{e}-06 \backslash \mathrm{Dipole}=-0.3544958,-0.0417535,0.694415$ $9 \backslash \mathrm{PG}=\mathrm{C} 01 \quad[\mathrm{X}(\mathrm{C} 3 \mathrm{H} 6 \mathrm{~S} 2)] \backslash \backslash @$

\section{$\cdot \mathrm{CH}_{3}$}

$1 \backslash 1 \backslash G I N C-S C 116 \backslash F O p t \backslash U B 3 L Y P \backslash 6-31 G(d) \backslash C 1 H 3(2) \backslash R G B 501 \backslash 22-A u g-2002 \backslash 0 \backslash \backslash \# N B$ 3LYP 6-31G(D) SCF=TIGHT OPT=TIGHT FREQ MAXDISK=13107200 \methyl opt\&Fr eq UB3-LYP $/ 6-31 \mathrm{G}(\mathrm{d}) \backslash \backslash 0,2 \backslash \mathrm{C}, 0 ., 0 ., 0 . \backslash \mathrm{H}, 1.082752351,0 ., 0 . \backslash \mathrm{H},-0.541376175$ $5,-0.937691042,0 . \backslash \mathrm{H},-0.5413761755,0.937691042,0 . \backslash \backslash$ Version=DEC-AXP-OSF / $1-G 98 R e v A .11 .3 \backslash \mathrm{HF}=-39.8382922 \backslash \mathrm{S} 2=0.753765 \backslash \mathrm{S} 2-1=0 . \backslash \mathrm{S} 2 \mathrm{~A}=0.750007 \backslash \mathrm{RMSD}=4$. $410 \mathrm{e}-09 \backslash \mathrm{RMSF}=1.426 \mathrm{e}-09 \backslash \mathrm{Dipole}=0 ., 0 ., 0 . \backslash \mathrm{PG}=\mathrm{D} 03 \mathrm{H} \quad[\mathrm{O}(\mathrm{C} 1), 3 \mathrm{C} 2(\mathrm{H} 1)] \backslash \backslash @$

\section{$\cdot \mathrm{CH}_{2} \mathrm{CH}_{3}$}

$1 \backslash 1 \backslash G I N C-S C 116 \backslash F O p t \backslash U B 3 L Y P \backslash 6-31 G(d) \backslash C 2 H 5(2) \backslash M L C 501 \backslash 06-M a r-2003 \backslash 0 \backslash \backslash \# N \quad B$

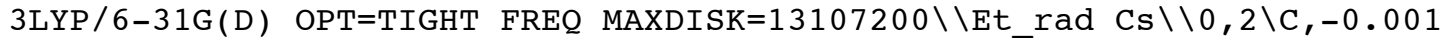
$4599287,0.7954773663,0 . \backslash \mathrm{C}, 0.0222526364,-0.6939020591,0 . \backslash \mathrm{H},-0.994810722$ $3,-1.1263631861,0 . \backslash \mathrm{H},-0.0929966331,1.3519648812,0.9274633127 \backslash \mathrm{H},-0.0929$ $966331,1.3519648812,-0.9274633127 \backslash \mathrm{H}, 0.5280238711,-1.0935092097,0.88764$ $34386 \backslash \mathrm{H}, 0.5280238711,-1.0935092097,-0.8876434386 \backslash \backslash$ Version=DEC-AXP-OSF / $1-G 98 R e v A .11 .3 \backslash$ State $=2-A^{\prime} \backslash \mathrm{HF}=-79.1578673 \backslash \mathrm{S} 2=0.753921 \backslash \mathrm{S} 2-1=0 . \backslash \mathrm{S} 2 \mathrm{~A}=0.750$ $01 \backslash \mathrm{RMSD}=8.620 \mathrm{e}-09 \backslash \mathrm{RMSF}=3.177 \mathrm{e}-06 \backslash \mathrm{Dipole}=-0.0504824,-0.0860887,0 . \backslash \mathrm{PG}=\mathrm{CS}$ $[\mathrm{SG}(\mathrm{C} 2 \mathrm{H} 1), \mathrm{X}(\mathrm{H} 4)] \backslash \backslash @$

\section{$\cdot \mathrm{CH}_{2} \mathrm{CN}$}

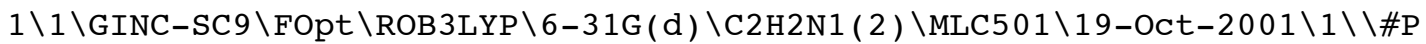

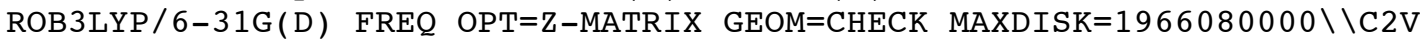
cyanomethy $1 \backslash \backslash 0,2 \backslash \mathrm{X} \backslash \mathrm{C}, 1,1 . \backslash \mathrm{N}, 2, \mathrm{CN}, 1,90 . \backslash \mathrm{C}, 2, \mathrm{CC}, 1,90 ., 3,180 ., 0 \backslash \mathrm{H}, 4, \mathrm{HC}, 2$, $\mathrm{HCC}, 1,180 ., 0 \backslash \mathrm{H}, 4, \mathrm{HC}, 2, \mathrm{HCC}, 1,0 ., 0 \backslash \backslash \mathrm{CN}=1.17367022 \backslash \mathrm{CC}=1.3865528 \backslash \mathrm{HC}=1.0836$ $2585 \backslash \mathrm{HCC}=120.29410501 \backslash \backslash$ Version=DEC-AXP-OSF $/ 1-G 98$ RevA. $9 \backslash$ State $=2-B 1 \backslash \mathrm{HF}=-$ $132.0915686 \backslash \mathrm{RMSD}=2.302 \mathrm{e}-09 \backslash \mathrm{RMSF}=1.601 \mathrm{e}-05 \backslash \mathrm{Dipole}=-1.3355252,0 ., 0 . \backslash \mathrm{PG}=\mathrm{C}$ $02 \mathrm{~V}[\mathrm{C} 2(\mathrm{C} 1 \mathrm{C} 1 \mathrm{~N} 1), \mathrm{SGV}(\mathrm{H} 2)] \backslash \backslash @$

\section{- $\mathrm{C}\left(\mathrm{CH}_{3}\right)_{2} \mathrm{CN}$}

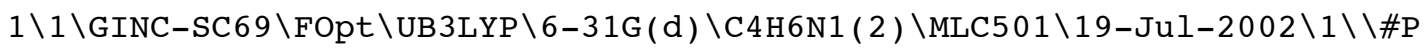
UB3LYP $/ 6-31 \mathrm{G} *$ FOPT $=(\mathrm{Z}-\mathrm{MATRIX}$, TIGHT, MAXCYC $=100)$ FREQ MAXDISK $=131072000$ $\mathrm{SCF}=(\mathrm{TIGHT}, \mathrm{MAXCYC}=100) \backslash \backslash \mathrm{me} 2 \mathrm{CCn}-\mathrm{C} 2 \mathrm{vb}-6 \mathrm{dub} 3 \backslash \backslash 0,2 \backslash \mathrm{C} \backslash \mathrm{C}, 1, \mathrm{~b} 1 \backslash \mathrm{X}, 2,1 ., 1,90 . \backslash \mathrm{N}$ $, 2, \mathrm{~b} 2,3,90 \ldots, 1,180 ., 0 \backslash \mathrm{C}, 1, \mathrm{~b} 3,2, \mathrm{a} 2,3,0 ., 0 \backslash \mathrm{C}, 1, \mathrm{~b} 3,2, \mathrm{a} 2,3,180 ., 0 \backslash \mathrm{H}, 5, \mathrm{~b} 4,1$, $\mathrm{a} 3,2,0 ., 0 \backslash \mathrm{H}, 6, \mathrm{~b} 4,1, \mathrm{a} 3,2,0 ., 0 \backslash \mathrm{H}, 5, \mathrm{~b} 5,1, \mathrm{a} 4,7, \mathrm{~d} 1,0 \backslash \mathrm{H}, 5, \mathrm{~b} 5,1, \mathrm{a} 4,7,-\mathrm{d} 1,0 \backslash \mathrm{H}$, $6, \mathrm{~b} 5,1, \mathrm{a} 4,8, \mathrm{~d} 1,0 \backslash \mathrm{H}, 6, \mathrm{~b} 5,1, \mathrm{a} 4,8,-\mathrm{d} 1,0 \backslash \backslash \mathrm{b} 1=1.39173287 \backslash \mathrm{b} 2=1.17666605 \backslash \mathrm{b} 3=1$ $.50156142 \backslash \mathrm{b} 4=1.09243341 \backslash \mathrm{b} 5=1.09976071 \backslash \mathrm{a} 2=120.24203494 \backslash \mathrm{a} 3=112.14994686 \backslash$ 
$\mathrm{a} 4=110.62200038 \backslash \mathrm{d} 1=121.09506187 \backslash \backslash$ Version=DEC-AXP-OSF $/ 1-G 98 \mathrm{RevA} .11 .3 \backslash \mathrm{St}$ ate $=2-\mathrm{B} 1 \backslash \mathrm{HF}=-210.7373225 \backslash \mathrm{S} 2=0.765669 \backslash \mathrm{S} 2-1=0 . \backslash \mathrm{S} 2 \mathrm{~A}=0.750113 \backslash \mathrm{RMSD}=8.850 \mathrm{e}-$ $09 \backslash \mathrm{RMSF}=1.667 \mathrm{e}-06 \backslash \mathrm{Dipole}=0 ., 0 .,-1.623572 \backslash \mathrm{PG}=\mathrm{C} 02 \mathrm{~V} \quad[\mathrm{C} 2(\mathrm{C} 1 \mathrm{C} 1 \mathrm{~N} 1), \mathrm{SGV}(\mathrm{C} 2 \mathrm{H} 2)$ , $\mathrm{X}(\mathrm{H} 4) \mathrm{l} \backslash \backslash \mathrm{Q}$

\section{$\cdot \mathrm{CH}_{2} \mathrm{COOCH}_{3}$}

$1 \backslash 1 \backslash G I N C-S C 98 \backslash F O p t \backslash U B 3 L Y P \backslash 6-31 G(d) \backslash C 3 H 5 O 2$ ( 2$) \backslash M L C 501 \backslash 28-J u n-2002 \backslash 1 \backslash \backslash \# U$ B3LYP /6-31G* FOPT $=(Z-M A T R I X, M A X C Y C=100$, TIGHT $)$ MAXDISK $=65536000$ FREQ SC $\mathrm{F}=(\mathrm{TIGHT}, \mathrm{MAXCYC}=100) \backslash \backslash \mathrm{ch} 2 \mathrm{cooch} 3 \mathrm{rad}(\mathrm{MA}) \mathrm{Cs} \backslash \backslash 0,2 \backslash \mathrm{C} \backslash \mathrm{C}, 1, \mathrm{~B} 1 \backslash 0,2, \mathrm{~B} 2,1, \mathrm{~A} 1 \backslash$

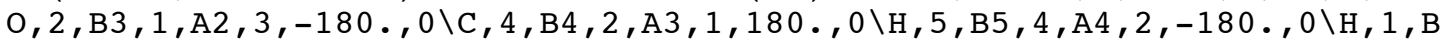
$6,2, \mathrm{~A} 5,3,180 ., 0 \backslash \mathrm{H}, 1, \mathrm{~B} 7,2, \mathrm{~A} 6,7,-180 ., 0 \backslash \mathrm{H}, 5, \mathrm{~B} 8,4, \mathrm{~A} 7,6, \mathrm{D} 1,0 \backslash \mathrm{H}, 5, \mathrm{~B} 8,4, \mathrm{~A} 7,6$ $,-D 1,0 \backslash \backslash \mathrm{B} 1=1.44732926 \backslash \mathrm{B} 2=1.22312018 \backslash \mathrm{B} 3=1.36100596 \backslash \mathrm{B} 4=1.43336725 \backslash \mathrm{B} 5=1.0$ $9058408 \backslash \mathrm{B} 6=1.0833186 \backslash \mathrm{B} 7=1.0824072 \backslash \mathrm{B} 8=1.09394961 \backslash \mathrm{A} 1=124.74743883 \backslash \mathrm{A} 2=111$ $.87082202 \backslash A 3=114.93235024 \backslash A 4=105.62119929 \backslash A 5=121.24789916 \backslash A 6=118.31516$ $036 \backslash \mathrm{A} 7=110.72623732 \backslash \mathrm{D} 1=119.68853085 \backslash \backslash$ Version=DEC-AXP-OSF $/ 1-\mathrm{G} 98 \mathrm{RevA} .11$. $3 \backslash$ State $=2-A^{\prime \prime} \backslash H F=-267.7237418 \backslash S 2=0.757594 \backslash S 2-1=0 . \backslash S 2 A=0.750034 \backslash \mathrm{RMSD}=4.6$ $63 e-09 \backslash \mathrm{RMSF}=3.942 \mathrm{e}-06 \backslash \mathrm{Dipole}=-0.6413843,0,,-0.2480326 \backslash \mathrm{PG}=\mathrm{CS} \quad[\mathrm{SG}(\mathrm{C} 3 \mathrm{H} 3 \mathrm{O} 2$ ) $\mathrm{X}(\mathrm{H} 2) \mathrm{]} \backslash \backslash \mathrm{Q}$

\section{$\cdot \mathrm{CH}\left(\mathrm{COOCH}_{3}\right) \mathrm{CH}_{3}$}

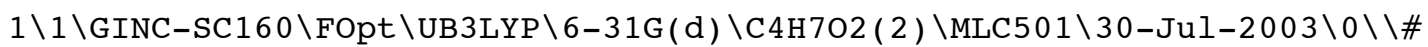
B3LYP/6-31G* OPT=TIGHT FREQ MAXDISK=39321600 GEOM=CHECK GUESS=READ \\MA

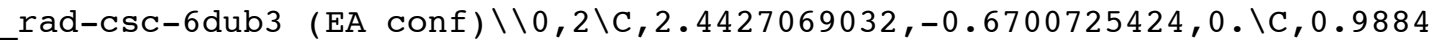
$\overline{4} 54995,-0.9680099437,0 . \backslash \mathrm{C}, 0.0000531191,0.0925714969,0 . \backslash 0,0.2492141423$, $1.2915528696,0 . \backslash 0,-1.2690884584,-0.4038514401,0 . \backslash C,-2.3055213085,0.584$ $7337423,0 . \backslash \mathrm{H},-3.2434106254,0.0277541879,0 . \backslash \mathrm{H}, 2.6133119038,0.4089560571$ , $0 . \backslash \mathrm{H}, 0.6334677625,-1.9941959598,0 . \backslash \mathrm{H}, 2.9379202621,-1.1090091687,0.878$ $9917495 \backslash \mathrm{H}, 2.9379202621,-1.1090091687,-0.8789917495 \backslash \mathrm{H},-2.2371601584,1.2$ $192780488,0.8886413064 \backslash \mathrm{H},-2.2371601584,1.2192780488,-0.8886413064 \backslash \backslash$ Ver sion=DEC-AXP-OSF / 1-G03RevB.03 \State=2-A" $\backslash \mathrm{HF}=-307.0484404 \backslash \mathrm{S} 2=0.756686 \backslash \mathrm{S}$ $2-1=0 . \backslash S 2 A=0.750029 \backslash \mathrm{RMSD}=9.256 \mathrm{e}-09 \backslash \mathrm{RMSF}=1.294 \mathrm{e}-06 \backslash \mathrm{Dipole}=-0.0936169,-0$ $.6405142,0 . \backslash \mathrm{PG}=\mathrm{CS} \quad[\mathrm{SG}(\mathrm{C} 4 \mathrm{H} 3 \mathrm{O} 2), \mathrm{X}(\mathrm{H} 4)] \backslash \backslash \mathrm{C}$

\section{$\cdot \mathrm{CH}_{2} \mathrm{OCOCH}_{3}$}

$1 \backslash 1 \backslash G I N C-S C 10 \backslash F O p t \backslash U B 3 L Y P \backslash 6-31 G(d) \backslash C 3 H 5 O 2$ ( 2$) \backslash M L C 501 \backslash 07-M a r-2003 \backslash 0 \backslash \backslash \# N$

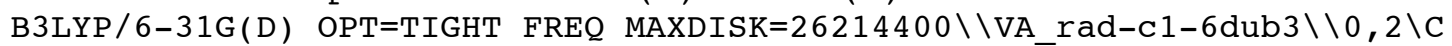
$,-1.7787811457,0.353773456,-0.0356590445 \backslash \mathrm{C},-0.2730471777,0.3716029248$, $0.0126778637 \backslash 0,0.4350619367,1.3462970692,0.0902212033 \backslash 0,0.2185047767,-$ $0.9118545746,-0.0469137652 \backslash \mathrm{H},-2.1541016217,1.3743705621,0.0441727311 \backslash \mathrm{C}$ $, 1.5760766477,-1.0845083545,-0.0455384982 \backslash \mathrm{H}, 2.202147411,-0.2279043038$, $0.1585273774 \backslash \mathrm{H}, 1.8766726719,-2.1166078394,0.0561765013 \backslash \mathrm{H},-2.1785837176$ $,-0.2564307074,0.7808932818 \backslash \mathrm{H},-2.1201583965,-0.0941758259,-0.975111322$ $\backslash \backslash$ Version=DEC-AXP-OSF / 1-G98RevA. $11.3 \backslash \mathrm{HF}=-267.7202621 \backslash \mathrm{S} 2=0.753529 \backslash \mathrm{S} 2-1=$ $0 . \backslash \mathrm{S} 2 \mathrm{~A}=0.750008 \backslash \mathrm{RMSD}=8.716 \mathrm{e}-09 \backslash \mathrm{RMSF}=1.382 \mathrm{e}-06 \backslash \mathrm{Dipole}=-0.4442854,-0.574$ $2031,0.0426497 \backslash P G=C 01[X(C 3 H 502)] \backslash \backslash @$

\section{$\cdot \mathrm{CH}_{2} \mathrm{Ph}$}

$1 \backslash 1 \backslash G I N C-S C 89 \backslash F O p t \backslash U B 3 L Y P \backslash 6-31 G(d) \backslash C 7 H 7(2) \backslash M L C 501 \backslash 18-J u 1-2002 \backslash 1 \backslash \backslash \#$ UB3 LYP $/ 6-31 \mathrm{G} * \mathrm{FOPT}=(\mathrm{TIGHT}, \mathrm{MAXCYC}=100, \mathrm{Z}-\mathrm{MATRIX})$ MAXDISK $=131072000$ FREQ SCF $=($ TIGHT, MAXCYC $=100) \backslash \backslash \mathrm{bz} \_r a d-c 2 v-6$ dub3 (guess from dave) $\backslash \backslash 0,2 \backslash C \backslash C, 1, B 1 \backslash$ $\mathrm{C}, 2, \mathrm{~B} 2,1, \mathrm{~A} 1 \backslash \mathrm{C}, 2, \mathrm{~B} 2,1, \mathrm{~A} 1,3,-180$, $0 \backslash \mathrm{C}, 3, \mathrm{~B} 3,2, \mathrm{~A} 2,1,-180$. $0 \backslash \mathrm{C}, 4, \mathrm{~B} 3,2, \mathrm{~A} 2,1$, $180 ., 0 \backslash \mathrm{H}, 1, \mathrm{~B} 4,2, \mathrm{~A} 3,3,0 ., 0 \backslash \mathrm{H}, 1, \mathrm{~B} 4,2, \mathrm{~A} 3,7,-180 ., 0 \backslash \mathrm{H}, 3, \mathrm{~B} 5,2, \mathrm{~A} 4,5,180 ., 0 \backslash \mathrm{H}$ , 4 , B5 , $2, \mathrm{~A} 4,6,-180 ., 0 \backslash \mathrm{H}, 5, \mathrm{~B} 6,3, \mathrm{~A} 5,2,180 ., 0 \backslash \mathrm{H}, 6, \mathrm{~B} 6,4, \mathrm{~A} 5,2,-180 ., 0 \backslash \mathrm{X}, 2,1$. $, 1,90 ., 3,90 ., 0 \backslash \mathrm{C}, 2, \mathrm{~B} 7,13,90.1,180 \ldots, 0 \backslash \mathrm{X}, 14,1 ., 2,90.13,0 \ldots, 0 \backslash \mathrm{H}, 14, \mathrm{~B} 8,15$ $, 90 ., 2,180 ., 0 \backslash \backslash \mathrm{B} 1=1.40658309 \backslash \mathrm{B} 2=1.4269787 \backslash \mathrm{B} 3=1.38585898 \backslash \mathrm{B} 4=1.08519274 \backslash$ $\mathrm{B} 5=1.08733663 \backslash \mathrm{B} 6=1.08707607 \backslash \mathrm{B} 7=2.83514342 \backslash \mathrm{B} 8=1.08628629 \backslash \mathrm{A} 1=121.3932742$ $5 \backslash A 2=121.13892253 \backslash A 3=121.22638869 \backslash A 4=118.71171974 \backslash A 5=119.70158608 \backslash \backslash$ Ver 
sion=DEC-AXP-OSF $/ 1-G 98 R$ RA $.11 .3 \backslash$ State $=2-B 1 \backslash \mathrm{HF}=-270.9151435 \backslash \mathrm{S} 2=0.783683$ $\backslash \mathrm{S} 2-1=0 . \backslash \mathrm{S} 2 \mathrm{~A}=0.750769 \backslash \mathrm{RMSD}=5.840 \mathrm{e}-09 \backslash \mathrm{RMSF}=5.706 \mathrm{e}-07 \backslash \mathrm{Dipole}=0 ., 0 ., 0.052$ $5474 \backslash \mathrm{PG}=\mathrm{C} 02 \mathrm{~V} \quad[\mathrm{C} 2(\mathrm{H} 1 \mathrm{C} 1 \mathrm{C} 1 \mathrm{C} 1), \mathrm{SGV}(\mathrm{C} 4 \mathrm{H} 6)] \backslash \backslash @$

\section{$\cdot \mathrm{CH}(\mathrm{Ph}) \mathrm{CH}_{3}$}

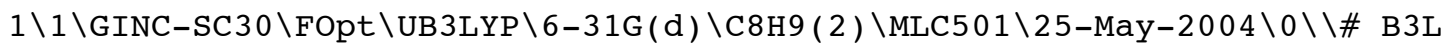

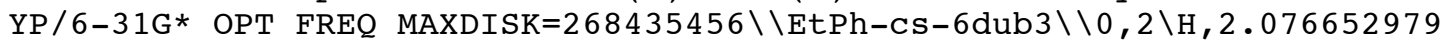
$1,-1.7071221701,0 . \backslash C, 1.8330785143,-0.6472341403,0 . \backslash C, 2.9648478287,0.33$ $29041964,0 . \backslash C, 0.4615044195,-0.296422334,0 . \backslash C,-2.3017509074,0.350177759$ $9,0 . \backslash C,-0.5414638247,-1.3098262805,0 . \backslash C, 0.01601966,1.0572057614,0 . \backslash C,-$ $1.3371627939,1.3659918739,0 . \backslash \mathrm{C},-1.8898621213,-0.9905058968,0 . \backslash \mathrm{H},-0.229$ $1124677,-2.3517513472,0 . \backslash \mathrm{H}, 0.7487305703,1.8588289809,0 . \backslash \mathrm{H},-1.649403422$ $1,2.4074639039,0 . \backslash \mathrm{H},-2.6317608755,-1.785156805,0 . \backslash \mathrm{H},-3.3593148857,0.59$ $85933381,0 . \backslash \mathrm{H}, 3.930913811,-0.179956881,0 . \backslash \mathrm{H}, 2.94101482,0.9926776704,0$. $8806011513 \backslash \mathrm{H}, 2.94101482,0.9926776704,-0.8806011513 \backslash \backslash$ Version=DEC-AXP-OS $\mathrm{F} / 1-\mathrm{G} 03$ RevB.03 $\backslash \mathrm{State}=2-\mathrm{A} " \backslash \mathrm{HF}=-310.2333536 \backslash \mathrm{S} 2=0.779917 \backslash \mathrm{S} 2-1=0 . \backslash \mathrm{S} 2 \mathrm{~A}=0.75$ $0613 \backslash \mathrm{RMSD}=8.432 \mathrm{e}-09 \backslash \mathrm{RMSF}=3.493 \mathrm{e}-05 \backslash \mathrm{Dipole}=0.1484688,0.0260889,0 . \backslash \mathrm{PG}=\mathrm{CS}$ $[\mathrm{SG}(\mathrm{C} 8 \mathrm{H} 7), \mathrm{X}(\mathrm{H} 2)] \backslash \backslash @$

\section{$\cdot \mathrm{C}(\mathrm{Ph})\left(\mathrm{CH}_{3}\right)_{2}$}

$1 \backslash 1 \backslash G I N C-S C 28 \backslash F O p t \backslash U B 3 L Y P \backslash 6-31 G(d) \backslash C 9 H 11(2) \backslash M L C 501 \backslash 25-M a y-2004 \backslash 0 \backslash \backslash \#$ B3

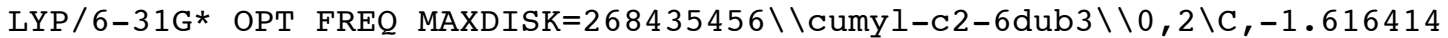
3386,0 . $0 . \backslash \mathrm{C},-0.1860957499,0.0 . \backslash \mathrm{C}, 0.5630674826,1.2120912698,0 . \backslash \mathrm{C}, 0.56$ $30674826,-1.2120912698,0 . \backslash \mathrm{C}, 1.9511243291,1.2069476466,0 . \backslash \mathrm{C}, 1.951124329$ $1,-1.2069476466,0 . \backslash \mathrm{H}, 0.0402217434,2.1628990039,0 . \backslash \mathrm{H}, 0.0402217434,-2.16$ $28990039,0 . \backslash \mathrm{H}, 2.4890058288,2.1518597904,0 . \backslash \mathrm{H}, 2.4890058288,-2.151859790$ $4,0 . \backslash \mathrm{C},-2.382682254,1.2955409885,0 . \backslash \mathrm{C},-2.382682254,-1.2955409885,0 . \backslash \mathrm{H}$, $-3.4618855224,1.1182105536,0 . \backslash \mathrm{H},-3.4618855224,-1.1182105536,0 . \backslash \mathrm{H},-2.15$ $20630813,1.9147870606,0.8799660511 \backslash \mathrm{H},-2.1520630813,-1.9147870606,0.879$ $9660511 \backslash \mathrm{H},-2.1520630813,1.9147870606,-0.8799660511 \backslash \mathrm{H},-2.1520630813,-1$. $9147870606,-0.8799660511 \backslash \mathrm{C}, 2.6605839324,0.0 . \backslash \mathrm{H}, 3.7470104688,0 ., 0 . \backslash \backslash \mathrm{Ve}$ rsion=DEC-AXP-OSF $/ 1-G 03 R e v B .03 \backslash$ State $=2-B 1 \backslash H F=-349.5492998 \backslash \mathrm{S} 2=0.775843 \backslash$ $\mathrm{S} 2-1=0 . \backslash \mathrm{S} 2 \mathrm{~A}=0.750464 \backslash \mathrm{RMSD}=4.694 \mathrm{e}-09 \backslash \mathrm{RMSF}=4.133 \mathrm{e}-05 \backslash \mathrm{Dipole}=-0.2539911,0$ , $0 . \backslash \mathrm{PG}=\mathrm{C} 02 \mathrm{~V} \quad[\mathrm{C} 2(\mathrm{C} 1 \mathrm{C} 1 \mathrm{C} 1 \mathrm{H} 1), \mathrm{SGV}(\mathrm{C} 6 \mathrm{H} 6), \mathrm{X}(\mathrm{H} 4)] \backslash \backslash @$ 
Table S2. Geometries of the Thiocarbonyl Products $\left(\mathrm{R}^{\prime} \mathrm{SC}\left(\mathrm{CH}_{3}\right)=\mathrm{S}\right)$ of Beta-Scission Used in Calculating the Beta-Scission Enthalpies in Table 2

\author{
$\mathrm{S}=\mathrm{C}\left(\mathrm{CH}_{3}\right) \mathrm{S}-\mathrm{CH}_{2} \mathrm{CH}_{3}$ \\ $1 \backslash 1 \backslash G I N C-S C 32 \backslash F O p t \backslash R B 3 L Y P \backslash 6-31 G(d) \backslash C 4 H 8 S 2 \backslash M L C 501 \backslash 09-S e p-2004 \backslash 0 \backslash \backslash \#$ B3LY

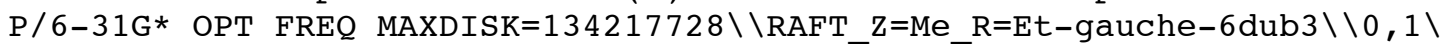 \\ $C,-0.2021164069,-0.9585999653,0.258035939 \overline{9} \backslash \mathrm{S}, 1.4145758914,-1.016291554$ \\ $5,0.5661635493 \backslash \mathrm{S},-1.1663336787,0.4841880056,-0.0245889377 \backslash \mathrm{C},-1.0554120$ \\ $918,-2.2064011252,0.1588030295 \backslash \mathrm{C}, 0.0317386338,1.8716959479,0.025188402$ \\ $1 \backslash \mathrm{H}, 0.7621890764,1.6304000812,0.8031614027 \backslash \mathrm{H},-0.5607800252,2.730748178$ \\ $6,0.3558726122 \backslash \mathrm{C}, 0.7126329915,2.1422063412,-1.3151105562 \backslash \mathrm{H},-0.73052527$ \\ $77,-2.9376778743,0.9027370235 \backslash \mathrm{H},-0.9154878523,-2.6552018848,-0.8323008$ \\ $996 \backslash \mathrm{H},-2.1231488196,-1.9992885005,0.2892886971 \backslash \mathrm{H}, 1.3040551345,1.279272$ \\ $2712,-1.6327838722 \backslash \mathrm{H}, 1.3896069091,2.9993255,-1.2165503967 \backslash \mathrm{H},-0.0188433$ \\ $086,2.3726718201,-2.096119244 \backslash \backslash$ Version=DEC-AXP-OSF /1-G03RevB.03 $\backslash$ State $=$ \\ $1-\mathrm{A} \backslash \mathrm{HF}=-953.621634 \backslash \mathrm{RMSD}=3.065 e-09 \backslash \mathrm{RMSF}=8.508 \mathrm{e}-06 \backslash \mathrm{Dipole}=-0.6099368,0.4$ \\ $058309,-0.2534361 \backslash \mathrm{PG}=\mathrm{C} 01 \quad[\mathrm{X}(\mathrm{C} 4 \mathrm{H} 8 \mathrm{~S} 2)] \backslash \backslash @$
}

\title{
$\mathrm{S}=\mathrm{C}\left(\mathrm{CH}_{3}\right) \mathrm{S}-\mathrm{CH}_{2} \mathrm{CN}$
}

$1 \backslash 1 \backslash G I N C-S C 45 \backslash F O p t \backslash R B 3 L Y P \backslash 6-31 G(d) \backslash C 4 H 5 N 1 S 2 \backslash M L C 501 \backslash 19-M a r-2004 \backslash 1 \backslash \backslash \#$ B3 $\mathrm{LYP} / 6-31 \mathrm{G} * \mathrm{FOPT}=(\mathrm{MAXCYC}=300, \mathrm{Z}-\mathrm{MATRIX})$ MAXDISK $=134217728 \backslash \backslash \mathrm{RAFT} Z \mathrm{Z}=\mathrm{CH} 3 \_\mathrm{R}=$ $\mathrm{CH} 2 \mathrm{CN}-\mathrm{a} 3 \mathrm{~b} 1-6 \mathrm{dub} 3 \backslash \backslash 0,1 \backslash \mathrm{C} \backslash \mathrm{C}, 1, \mathrm{~B} 1 \backslash \mathrm{S}, 2, \mathrm{~B} 2,1, \mathrm{~A} 1 \backslash \mathrm{S}, 2, \mathrm{~B} 3,1, \mathrm{~A} 2,3, \mathrm{D} 1,0 \backslash \mathrm{C}, 4, \mathrm{~B} 4,2$ , $\mathrm{A} 3,1, \mathrm{D} 2,0 \backslash \mathrm{C}, 5, \mathrm{~B} 5,4, \mathrm{~A} 4,2, \mathrm{D} 3,0 \backslash \mathrm{H}, 5, \mathrm{~B} 6,4, \mathrm{~A} 5,6, \mathrm{D} 4,0 \backslash \mathrm{H}, 5, \mathrm{~B} 7,4, \mathrm{~A} 6,6, \mathrm{D} 5,0 \backslash \mathrm{H}$, $1, \mathrm{~B} 8,2, \mathrm{~A} 7,3, \mathrm{D} 6,0 \backslash \mathrm{H}, 1, \mathrm{~B} 9,2, \mathrm{~A} 8,9, \mathrm{D} 7,0 \backslash \mathrm{H}, 1, \mathrm{~B} 10,2, \mathrm{~A} 9,9, \mathrm{D} 8,0 \backslash \mathrm{X}, 6,1 ., 5,90 ., 4$ , $\mathrm{D} 9,0 \backslash \mathrm{N}, 6, \mathrm{~B} 11,12, \mathrm{~A} 10,5,180 ., 0 \backslash \backslash \mathrm{B} 1=1.51416247 \backslash \mathrm{B} 2=1.64284125 \backslash \mathrm{B} 3=1.761076$ $58 \backslash B 4=1.8403095 \backslash B 5=1.45879272 \backslash B 6=1.09433674 \backslash B 7=1.09441266 \backslash B 8=1.0954722$ $4 \backslash B 9=1.09682466 \backslash B 10=1.09245527 \backslash B 11=1.16042021 \backslash A 1=123.37991907 \backslash A 2=110.7$ $4648554 \backslash A 3=102.0502169 \backslash A 4=109.36720461 \backslash A 5=109.0929812 \backslash A 6=108.97689249 \backslash$ $\mathrm{A} 7=112.7778099 \backslash \mathrm{A} 8=108.89778025 \backslash \mathrm{A} 9=109.71152501 \backslash \mathrm{A} 10=89.81567531 \backslash \mathrm{D} 1=-178$ $.57873247 \backslash D 2=177.17630707 \backslash D 3=-180.48291408 \backslash D 4=121.33196566 \backslash D 5=-121.292$ $88244 \backslash D 6=-160.12420209 \backslash D 7=-119.9522014 \backslash D 8=122.30311959 \backslash D 9=-171.3058611$ $\backslash \backslash$ Version=DEC-AXP-OSF $/ 1-G 03 R e v B .03 \backslash$ State $=1-A \backslash H F=-1006.5367763 \backslash \mathrm{RMSD}=2.5$ $18 e-09 \backslash \mathrm{RMSF}=2.636 \mathrm{e}-05 \backslash \mathrm{Dipole}=0.6227997,-0.0165881,-1.0213479 \backslash \mathrm{PG}=\mathrm{C} 01] \mathrm{X}$ ( C4H5N1S2) ] \\@

\section{$\mathrm{S}=\mathrm{C}\left(\mathrm{CH}_{3}\right) \mathrm{S}-\mathrm{C}\left(\mathrm{CH}_{3}\right)_{2} \mathrm{CN}$}

$1 \backslash 1 \backslash G I N C-S C 70 \backslash F O p t \backslash R B 3 L Y P \backslash 6-31 G(d) \backslash C 6 H 9 N 1 S 2 \backslash M L C 501 \backslash 01-A u g-2002 \backslash 1 \backslash \backslash \# N B$ 3LYP $/ 6-31 \mathrm{G} * \mathrm{OPT}=(\mathrm{Z}-\mathrm{MATRIX}, \mathrm{MAXCYC}=100) \mathrm{SCF}=(\mathrm{TIGHT}, \mathrm{MAXCYC}=100)$ FREQ MAXD ISK $=131072000 \backslash \backslash \operatorname{RAFT}(\mathrm{Z}=\mathrm{Me} \mathrm{R}=\mathrm{Me} 2 \mathrm{CCN}) \mathrm{c} 1 \mathrm{x} / / \mathrm{B} 3 \backslash \backslash 0,1 \backslash \mathrm{S} \backslash \mathrm{C}, 1, \mathrm{~B} 1 \backslash \mathrm{S}, 2, \mathrm{~B} 2,1, \mathrm{~A} 1 \backslash \mathrm{C}$ , 2 , B3 , $1, \mathrm{~A} 2,3, \mathrm{D} 1,0 \backslash \mathrm{H}, 4, \mathrm{~B} 4,2, \mathrm{~A} 3,1, \mathrm{D} 2,0 \backslash \mathrm{H}, 4, \mathrm{~B} 5,2, \mathrm{~A} 4,5, \mathrm{D} 3,0 \backslash \mathrm{H}, 4, \mathrm{~B} 6,2, \mathrm{~A} 5,5$, $\mathrm{D} 4,0 \backslash \mathrm{C}, 3, \mathrm{~B} 7,2, \mathrm{~A} 6,1, \mathrm{D} 5,0 \backslash \mathrm{C}, 8, \mathrm{~B} 8,3, \mathrm{~A} 7,2, \mathrm{D} 6,0 \backslash \mathrm{C}, 8, \mathrm{~B} 9,3, \mathrm{~A} 8,2, \mathrm{D} 7,0 \backslash \mathrm{C}, 8, \mathrm{~B} 10$, $3, \mathrm{~A} 9,2, \mathrm{D} 8,0 \backslash \mathrm{H}, 9, \mathrm{~B} 11,8, \mathrm{~A} 10,3, \mathrm{D} 9,0 \backslash \mathrm{H}, 9, \mathrm{~B} 12,8, \mathrm{~A} 11,12, \mathrm{D} 10,0 \backslash \mathrm{H}, 9, \mathrm{~B} 13,8, \mathrm{~A} 12$, $12, \mathrm{D} 11,0 \backslash \mathrm{H}, 10, \mathrm{~B} 14,8, \mathrm{~A} 13,3, \mathrm{D} 12,0 \backslash \mathrm{H}, 10, \mathrm{~B} 15,8, \mathrm{~A} 14,15, \mathrm{D} 13,0 \backslash \mathrm{H}, 10, \mathrm{~B} 16,8, \mathrm{~A} 15$ $, 15, \mathrm{D} 14,0 \backslash \mathrm{X}, 11,1 ., 8,90 ., 3, \mathrm{D} 15,0 \backslash \mathrm{N}, 11, \mathrm{~B} 17,18, \mathrm{~A} 16,8,180 ., 0 \backslash \backslash \mathrm{D} 5=-2.820948$ $49 \backslash D 6=-172.62404781 \backslash D 7=68.00817564 \backslash D 8=-56.90651482 \backslash B 1=1.63879425 \backslash B 2=1$. $76680407 \backslash \mathrm{B} 3=1.51664757 \backslash \mathrm{B} 4=1.09173812 \backslash \mathrm{B} 5=1.09729372 \backslash \mathrm{B} 6=1.09598333 \backslash \mathrm{B} 7=1$. $87433331 \backslash \mathrm{B} 8=1.54722314 \backslash \mathrm{B} 9=1.53898597 \backslash \mathrm{B} 10=1.46987509 \backslash \mathrm{B} 11=1.09456438 \backslash \mathrm{B} 12$ $=1.09322437 \backslash \mathrm{B} 13=1.09446459 \backslash \mathrm{B} 14=1.09409064 \backslash \mathrm{B} 15=1.09027307 \backslash \mathrm{B} 16=1.0958685$ $8 \backslash \mathrm{B} 17=1.16134755 \backslash \mathrm{A} 1=127.88351466 \backslash \mathrm{A} 2=122.84831019 \backslash \mathrm{A} 3=109.71857276 \backslash \mathrm{A} 4=10$ $8.90699869 \backslash A 5=112.61761734 \backslash A 6=109.27146747 \backslash A 7=103.67051966 \backslash A 8=112.0180$ $1241 \backslash A 9=110.13118858 \backslash A 10=110.03666299 \backslash A 11=111.60473945 \backslash A 12=109.0263257$ $8 \backslash A 13=109.37095779 \backslash A 14=111.39024386 \backslash A 15=109.21696336 \backslash A 16=85.4520307 \backslash D 1$ $=182.8180857 \backslash \mathrm{D} 2=-29.5384385 \backslash \mathrm{D} 3=118.24218125 \backslash \mathrm{D} 4=-122.31452243 \backslash \mathrm{D} 9=-60.96$ $814176 \backslash \mathrm{D} 10=121.21780839 \backslash \mathrm{D} 11=-118.90528497 \backslash \mathrm{D} 12=57.82202549 \backslash \mathrm{D} 13=-121.128$ $30858 \backslash \mathrm{D} 14=118.88269266 \backslash \mathrm{D} 15=-130.36793124 \backslash \backslash$ Version=DEC-AXP-OSF $/ 1-\mathrm{G} 98 \mathrm{ReV}$ 
A. 11.3\HF=-1085.1615945 \RMSD=2.504e-09 $\backslash \mathrm{RMSF}=4.958 \mathrm{e}-05 \backslash \mathrm{Dipole}=0.2137102$ $, 1.1141232,1.4101622 \backslash \mathrm{PG}=\mathrm{C} 01 \quad[\mathrm{X}(\mathrm{C} 6 \mathrm{H} 9 \mathrm{~N} 1 \mathrm{~S} 2)] \backslash \backslash @$

\section{$\mathrm{S}=\mathrm{C}\left(\mathrm{CH}_{3}\right) \mathrm{S}-\mathrm{CH}_{2} \mathrm{COOCH}_{3}$}

$1 \backslash 1 \backslash \mathrm{GINC}-\mathrm{SC} 38 \backslash \mathrm{FOpt} \backslash \mathrm{RB} 3 L \mathrm{LP} \backslash 6-31 \mathrm{G}(\mathrm{d}) \backslash \mathrm{C} 5 \mathrm{H} 802 \mathrm{~S} 2 \backslash \mathrm{MLC} 501 \backslash 01-\mathrm{Aug}-2002 \backslash 1 \backslash \backslash \# \mathrm{~N} B$ 3LYP $/ 6-31 \mathrm{G} * \mathrm{OPT}=(\mathrm{Z}-\mathrm{MATRIX}, \mathrm{MAXCYC}=100) \mathrm{SCF}=(\mathrm{TIGHT}, \mathrm{MAXCYC}=100)$ FREQ MAXD $\mathrm{ISK}=131072000 \backslash \backslash \mathrm{RAFT}(\mathrm{Z}=\mathrm{Me} \mathrm{R}=\mathrm{MA}) \quad(\mathrm{c} 1 \mathrm{~b}) / / \mathrm{B} 3 \backslash \backslash 0,1 \backslash \mathrm{C} \backslash \mathrm{S}, 1, \mathrm{~B} 1 \backslash \mathrm{C}, 1, \mathrm{~B} 2,2, \mathrm{~A} 1 \backslash \mathrm{S}, 1$ , $\mathrm{B} 3,2, \mathrm{~A} 2,3, \mathrm{D} 1,0 \backslash \mathrm{H}, 3, \mathrm{~B} 4,1, \mathrm{~A} 3,2, \mathrm{D} 2,0 \backslash \mathrm{H}, 3, \mathrm{~B} 5,1, \mathrm{~A} 4,5, \mathrm{D} 3,0 \backslash \mathrm{H}, 3, \mathrm{~B} 6,1, \mathrm{~A} 5,5, \mathrm{D} 4$ , $0 \backslash \mathrm{C}, 4, \mathrm{~B} 7,1, \mathrm{~A} 6,2$, $\mathrm{D} 5,0 \backslash \mathrm{H}, 8, \mathrm{~B} 8,4, \mathrm{~A} 7,1, \mathrm{D} 6,0 \backslash \mathrm{H}, 8, \mathrm{~B} 9,4, \mathrm{~A} 8,1, \mathrm{D} 7,0 \backslash \mathrm{C}, 8, \mathrm{~B} 10,4$, $\mathrm{A} 9,1, \mathrm{D} 8,0 \backslash 0,11, \mathrm{~B} 11,8, \mathrm{~A} 10,4, \mathrm{D} 9,0 \backslash 0,11, \mathrm{~B} 12,8, \mathrm{~A} 11,12, \mathrm{D} 10,0 \backslash \mathrm{C}, 13, \mathrm{~B} 13,11, \mathrm{~A} 1$ $2,8, \mathrm{D} 11,0 \backslash \mathrm{H}, 14, \mathrm{~B} 14,13, \mathrm{~A} 13,11, \mathrm{D} 12,0 \backslash \mathrm{H}, 14, \mathrm{~B} 15,13, \mathrm{~A} 14,15, \mathrm{D} 13,0 \backslash \mathrm{H}, 14, \mathrm{~B} 16,1$ $3, \mathrm{~A} 15,15, \mathrm{D} 14,0 \backslash \backslash \mathrm{D} 5=0.94844174 \backslash \mathrm{D} 6=-58.07506369 \backslash \mathrm{D} 8=-179.56933226 \backslash \mathrm{D} 7=58.8$ $8551652 \backslash \mathrm{D} 9=0.05878835 \backslash \mathrm{B} 1=1.64764965 \backslash \mathrm{B} 2=1.51577468 \backslash \mathrm{B} 3=1.75399464 \backslash \mathrm{B} 4=1.0$ $9537429 \backslash \mathrm{B} 5=1.09622663 \backslash \mathrm{B} 6=1.09314218 \backslash \mathrm{B} 7=1.82281113 \backslash \mathrm{B} 8=1.09427601 \backslash \mathrm{B} 9=1.0$ $9424892 \backslash \mathrm{B} 10=1.51743154 \backslash \mathrm{B} 11=1.21071195 \backslash \mathrm{B} 12=1.34556723 \backslash \mathrm{B} 13=1.44048522 \backslash \mathrm{B} 1$ $4=1.09284572 \backslash B 15=1.09286824 \backslash B 16=1.08984645 \backslash A 1=122.48783328 \backslash A 2=126.6156$ $9031 \backslash \mathrm{A} 3=112.87807881 \backslash \mathrm{A} 4=109.00443803 \backslash \mathrm{A} 5=109.63960531 \backslash \mathrm{A} 6=102.40049134 \backslash \mathrm{A}$ $7=109.90359199 \backslash A 8=110.01261947 \backslash A 9=109.5060228 \backslash A 10=125.78683417 \backslash A 11=109$ $.75088779 \backslash A 12=115.36010348 \backslash A 13=110.41129286 \backslash A 14=110.40521778 \backslash A 15=105.3$ $9473697 \backslash \mathrm{D} 1=-178.78732782 \backslash \mathrm{D} 2=167.28801829 \backslash \mathrm{D} 3=120.39420154 \backslash \mathrm{D} 4=-122.15103$ $052 \backslash D 10=180.00429846 \backslash D 11=-179.98706551 \backslash D 12=-60.21919628 \backslash D 13=120.770316$ $57 \backslash \mathrm{D} 14=-119.62267623 \backslash \backslash$ Version=DEC-AXP-OSF $/ 1-G 98 R e v A .11 .3 \backslash \mathrm{HF}=-1142.1744$ $019 \backslash \mathrm{RMSD}=5.217 \mathrm{e}-09 \backslash \mathrm{RMSF}=1.529 \mathrm{e}-05 \backslash \mathrm{Dipole}=-0.6553583,0.0612289,-0.13796$ $73 \backslash P G=C 01 \quad[X(C 5 H 802 S 2)] \backslash \backslash @$

\section{$\mathrm{S}=\mathrm{C}\left(\mathrm{CH}_{3}\right) \mathrm{S}-\mathrm{CH}\left(\mathrm{COOCH}_{3}\right) \mathrm{CH}_{3}$}

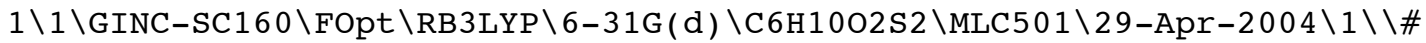
B3LYP $/ 6-31 \mathrm{G} * \mathrm{OPT}=(\mathrm{MAXCYC}=300, \mathrm{Z}-\mathrm{MATRIX})$ MAXDISK $=402653184 \mathrm{GEOM}=\mathrm{CHECK} \mathrm{GU}$ $\mathrm{ESS}=\mathrm{READ} \backslash \backslash \mathrm{RAFT} \mathrm{Z}=\mathrm{CH} 3 \_\mathrm{R}=\mathrm{MA}-\mathrm{a} 1 \mathrm{~b} 2 \mathrm{c} 1-6 \mathrm{dub} 3 \backslash \backslash 0,1 \backslash \mathrm{C} \backslash \mathrm{C}, 1, \mathrm{~B} 1 \backslash \mathrm{S}, 2, \mathrm{~B} 2,1, \mathrm{~A} 1 \backslash \mathrm{S}, 2, \mathrm{~B}$ $3,1, \mathrm{~A} 2,3, \mathrm{D} 1,0 \backslash \overline{\mathrm{C}}, 4, \mathrm{~B} 4,2, \mathrm{~A} 3,1, \mathrm{D} 2,0 \backslash \mathrm{C}, 5, \mathrm{~B} 5,4, \mathrm{~A} 4,2, \mathrm{D} 3,0 \backslash 0,6, \mathrm{~B} 6,5, \mathrm{~A} 5,4, \mathrm{D} 4,0$ $\backslash 0,6, \mathrm{~B} 7,5, \mathrm{~A} 6,7, \mathrm{D} 5,0 \backslash \mathrm{C}, 8, \mathrm{~B} 8,6, \mathrm{~A} 7,5, \mathrm{D} 6,0 \backslash \mathrm{H}, 1, \mathrm{~B} 9,2, \mathrm{~A} 8,3, \mathrm{D} 7,0 \backslash \mathrm{H}, 1, \mathrm{~B} 10,2, \mathrm{~A} 9$ $, 10, \mathrm{D} 8,0 \backslash \mathrm{H}, 1, \mathrm{~B} 11,2, \mathrm{~A} 10,10, \mathrm{D} 9,0 \backslash \mathrm{C}, 5, \mathrm{~B} 12,4, \mathrm{~A} 11,6, \mathrm{D} 10,0 \backslash \mathrm{H}, 5, \mathrm{~B} 13,4, \mathrm{~A} 12,6, \mathrm{D}$ $11,0 \backslash \mathrm{H}, 13, \mathrm{~B} 14,5, \mathrm{~A} 13,4, \mathrm{D} 12,0 \backslash \mathrm{H}, 13, \mathrm{~B} 15,5, \mathrm{~A} 14,15, \mathrm{D} 13,0 \backslash \mathrm{H}, 13, \mathrm{~B} 16,5, \mathrm{~A} 15,15$, $\mathrm{D} 14,0 \backslash \mathrm{H}, 9, \mathrm{~B} 17,8, \mathrm{~A} 16,6, \mathrm{D} 15,0 \backslash \mathrm{H}, 9, \mathrm{~B} 18,8, \mathrm{~A} 17,18, \mathrm{D} 16,0 \backslash \mathrm{H}, 9, \mathrm{~B} 19,8, \mathrm{~A} 18,18, \mathrm{D} 1$ $7,0 \backslash \backslash \mathrm{B} 1=1.51550401 \backslash \mathrm{B} 2=1.64433181 \backslash \mathrm{B} 3=1.76134063 \backslash \mathrm{B} 4=1.84857026 \backslash \mathrm{B} 5=1.5251$ $7796 \backslash B 6=1.21136371 \backslash B 7=1.3462955 \backslash B 8=1.43860754 \backslash B 9=1.09526915 \backslash B 10=1.0929$ $9943 \backslash B 11=1.09643129 \backslash B 12=1.52682847 \backslash B 13=1.09514382 \backslash B 14=1.09584525 \backslash B 15=1$ $.09251016 \backslash B 16=1.09306812 \backslash B 17=1.09315679 \backslash B 18=1.09302677 \backslash B 19=1.08997545 \backslash$ $\mathrm{A} 1=122.50208778 \backslash \mathrm{A} 2=110.33614943 \backslash \mathrm{A} 3=104.50918592 \backslash \mathrm{A} 4=107.0167987 \backslash \mathrm{A} 5=124$. $676591 \backslash \mathrm{A} 6=111.0790365 \backslash \mathrm{A} 7=115.43512528 \backslash \mathrm{A} 8=112.8464058 \backslash \mathrm{A} 9=109.64544647 \backslash \mathrm{A}$ $10=108.93490901 \backslash A 11=111.82345567 \backslash A 12=104.59673725 \backslash A 13=109.68963821 \backslash A 14$ $=111.04512925 \backslash \mathrm{A} 15=110.13224412 \backslash \mathrm{A} 16=110.44524996 \backslash \mathrm{A} 17=110.45853986 \backslash \mathrm{A} 18=1$ $05.45871601 \backslash D 1=181.46329095 \backslash D 2=-183.21978603 \backslash D 3=141.11637645 \backslash D 4=99.153$ $67554 \backslash D 5=179.83858035 \backslash D 6=-181.69244048 \backslash D 7=194.84412788 \backslash D 8=122.26999047$ $\backslash \mathrm{D} 9=-120.21567462 \backslash \mathrm{D} 10=123.25466656 \backslash \mathrm{D} 11=-115.97424246 \backslash \mathrm{D} 12=178.0396616 \backslash \mathrm{D}$ $13=119.28305957 \backslash D 14=-119.25449746 \backslash D 15=-60.33898082 \backslash D 16=120.74389688 \backslash D 1$ $7=-119.61072199 \backslash \backslash$ Version=DEC-AXP-OSF $/ 1-G 03$ RevB . 03 $\backslash$ State $=1-\mathrm{A} \backslash \mathrm{HF}=-1181.4$ $894033 \backslash \mathrm{RMSD}=7.435 \mathrm{e}-09 \backslash \mathrm{RMSF}=9.392 \mathrm{e}-06 \backslash \mathrm{Dipole}=-0.2931424,0.4343127,-0.30$ $83545 \backslash \mathrm{PG}=\mathrm{C} 01 \quad[\mathrm{X}(\mathrm{C} 6 \mathrm{H} 10 \mathrm{O} 2 \mathrm{~S} 2)] \backslash \backslash \mathrm{Q}$

\section{$\mathrm{S}=\mathrm{C}\left(\mathrm{CH}_{3}\right) \mathrm{S}-\mathrm{CH}_{2} \mathrm{OCOCH}_{3}$}

$1 \backslash 1 \backslash G I N C-S C 53 \backslash F O p t \backslash R B 3 L Y P \backslash 6-31 G(d) \backslash C 5 H 802 S 2 \backslash M L C 501 \backslash 02-J u 1-2004 \backslash 0 \backslash \backslash \#$ B3

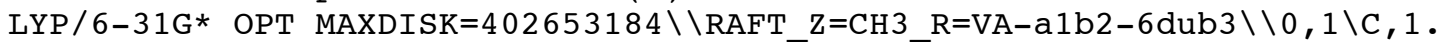
$9750593402,-0.6906704407,-2.5154211589 \backslash \mathrm{C}, 1.6 \overline{5} 01348913,-0.3029784604,-1$ $.0888895847 \backslash \mathrm{S}, 2.5261990151,-0.8251269234,0.1978201485 \backslash \mathrm{S}, 0.2070731555,0$ $.7246752909,-1.0510405555 \backslash \mathrm{C},-0.0960244756,0.9973156776,0.7317910752 \backslash \mathrm{H}$, $-0.5421795366,1.9890352195,0.814228878 \backslash \mathrm{H}, 0.8585272373,0.9210688293,1.2$ 
$529717273 \backslash 0,-0.9449297301,0.0155364685,1.3138958331 \backslash \mathrm{C},-2.2843842972,0$. $1892285971,1.1096864178 \backslash 0,-2.7428266425,1.1093549691,0.4732918047 \backslash \mathrm{C},-3$ $.0676524973,-0.9120390345,1.77686461 \backslash \mathrm{H},-4.1345473332,-0.7213776112,1.6$ $579717408 \backslash \mathrm{H},-2.8098780208,-1.8752537354,1.3237754242 \backslash \mathrm{H},-2.8100739776,-$ $0.97181762,2.8389061523 \backslash \mathrm{H}, 1.3996096212,-1.5869036431,-2.7809516196 \backslash \mathrm{H}, 3$ $.0364300065,-0.9284575118,-2.6072132954 \backslash \mathrm{H}, 1.7090104864,0.0966626578,-3$ $.2298517545 \backslash \backslash$ Version=DEC-AXP-OSF / 1-G03RevB.03 \State $=1-\mathrm{A} \backslash \mathrm{HF}=-1142.17846$ $68 \backslash \mathrm{RMSD}=3.338 \mathrm{e}-09 \backslash \mathrm{RMSF}=3.386 \mathrm{e}-06 \backslash \mathrm{Dipole}=-0.2392341,-0.2456155,-0.07087$ $47 \backslash \mathrm{PG}=\mathrm{C} 01 \quad[\mathrm{X}(\mathrm{C} 5 \mathrm{H} 8 \mathrm{O} 2 \mathrm{~S} 2)] \backslash \backslash @$

\section{$\mathrm{S}=\mathrm{C}\left(\mathrm{CH}_{3}\right) \mathrm{S}-\mathrm{CH}_{2} \mathrm{Ph}$}

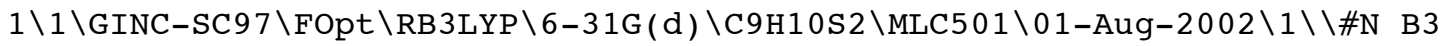
LYP $/ 6-31 \mathrm{G} * \mathrm{OPT}=(\mathrm{Z}-\mathrm{MATRIX}, \mathrm{MAXCYC}=100) \mathrm{SCF}=(\mathrm{TIGHT}, \mathrm{MAXCYC}=100)$ FREQ MAXDI $\mathrm{SK}=131072000 \backslash \backslash \mathrm{RAFT}(\mathrm{Z}=\mathrm{Me} \mathrm{R}=\mathrm{Bz}) \quad(\mathrm{C} 1 \mathrm{~b}) / / \mathrm{B} 3 \backslash \backslash 0,1 \backslash \mathrm{C} \backslash \mathrm{C}, 1, \mathrm{~B} 1 \backslash \mathrm{S}, 2, \mathrm{~B} 2,1, \mathrm{~A} 1 \backslash \mathrm{S}, 2$, $\mathrm{B} 3,1, \mathrm{~A} 2,3, \mathrm{D} 1,0 \backslash \mathrm{H}, 1, \mathrm{~B} 4,2, \mathrm{~A} 3,3, \mathrm{D} 2,0 \backslash \mathrm{H}, 1, \mathrm{~B} 5,2, \mathrm{~A} 4,5, \mathrm{D} 3,0 \backslash \mathrm{H}, 1, \mathrm{~B} 6,2, \mathrm{~A} 5,5, \mathrm{D} 4$, $0 \backslash \mathrm{C}, 4, \mathrm{~B} 7,2, \mathrm{~A} 6,1, \mathrm{D} 5,0 \backslash \mathrm{H}, 8, \mathrm{~B} 8,4, \mathrm{~A} 7,2, \mathrm{D} 6,0 \backslash \mathrm{H}, 8, \mathrm{~B} 9,4, \mathrm{~A} 8,2, \mathrm{D} 7,0 \backslash \mathrm{C}, 8, \mathrm{~B} 10,4, \mathrm{~A}$ 9,2 , $\mathrm{D} 8,0 \backslash \mathrm{C}, 11, \mathrm{~B} 11,8, \mathrm{~A} 10,4, \mathrm{D} 9,0 \backslash \mathrm{C}, 12, \mathrm{~B} 12,11, \mathrm{~A} 11,8, \mathrm{D} 10,0 \backslash \mathrm{C}, 13, \mathrm{~B} 13,12, \mathrm{~A} 12$ $, 11, \mathrm{D} 11,0 \backslash \mathrm{C}, 14, \mathrm{~B} 14,13, \mathrm{~A} 13,12, \mathrm{D} 12,0 \backslash \mathrm{C}, 15, \mathrm{~B} 15,14, \mathrm{~A} 14,13, \mathrm{D} 13,0 \backslash \mathrm{H}, 12, \mathrm{~B} 16,1$ $1, \mathrm{~A} 15,13, \mathrm{D} 14,0 \backslash \mathrm{H}, 13, \mathrm{~B} 17,12, \mathrm{~A} 16,14, \mathrm{D} 15,0 \backslash \mathrm{H}, 14, \mathrm{~B} 18,13, \mathrm{~A} 17,15, \mathrm{D} 16,0 \backslash \mathrm{H}, 15$, $\mathrm{B} 19,14, \mathrm{~A} 18,16, \mathrm{D} 17,0 \backslash \mathrm{H}, 16, \mathrm{~B} 20,15, \mathrm{~A} 19,14, \mathrm{D} 18,0 \backslash \backslash \mathrm{B} 1=1.5157017 \backslash \mathrm{B} 2=1.647716$ $37 \backslash \mathrm{B} 3=1.75389067 \backslash \mathrm{B} 4=1.09284038 \backslash \mathrm{B} 5=1.0966942 \backslash \mathrm{B} 6=1.09541288 \backslash \mathrm{B} 7=1.8457545$ $\backslash \mathrm{B} 8=1.0931606 \backslash \mathrm{B} 9=1.09316751 \backslash \mathrm{B} 10=1.50746347 \backslash \mathrm{B} 11=1.40121331 \backslash \mathrm{B} 12=1.394897$ $19 \backslash B 13=1.39611654 \backslash B 14=1.39623894 \backslash B 15=1.39469152 \backslash B 16=1.0875463 \backslash B 17=1.08$ $666122 \backslash \mathrm{B} 18=1.08661577 \backslash \mathrm{B} 19=1.08665644 \backslash \mathrm{B} 20=1.08753726 \backslash \mathrm{A} 1=122.61338998 \backslash \mathrm{A} 2$ $=111.38063085 \backslash \mathrm{A} 3=109.7490219 \backslash \mathrm{A} 4=108.97753356 \backslash \mathrm{A} 5=112.74245768 \backslash \mathrm{A} 6=103.93$ $454389 \backslash A 7=107.17960794 \backslash A 8=107.35823942 \backslash A 9=109.69738626 \backslash A 10=120.6321987$ $\backslash A 11=120.62629464 \backslash A 12=120.08811792 \backslash A 13=119.7336831 \backslash A 14=120.08376027 \backslash A 1$ $5=119.47797368 \backslash \mathrm{A} 16=119.7770135 \backslash \mathrm{A} 17=120.13175524 \backslash \mathrm{A} 18=120.13385522 \backslash \mathrm{A} 19=1$ $19.90004087 \backslash \mathrm{D} 1=178.73317096 \backslash \mathrm{D} 2=40.71288545 \backslash \mathrm{D} 3=-117.59620812 \backslash \mathrm{D} 4=122.324$ $46394 \backslash D 5=-177.58137358 \backslash D 6=-56.38412839 \backslash D 8=-178.59900137 \backslash D 7=59.07784813$ $\backslash \mathrm{D} 9=-90.47341043 \backslash \mathrm{D} 10=-179.84643742 \backslash \mathrm{D} 11=-0.13726456 \backslash \mathrm{D} 12=0.01725165 \backslash \mathrm{D} 13=$ $-0.01747664 \backslash \mathrm{D} 14=-180.28619998 \backslash \mathrm{D} 15=-180.1759319 \backslash \mathrm{D} 16=179.82246162 \backslash \mathrm{D} 17=17$ $9.82557756 \backslash \mathrm{D} 18=179.86971903 \backslash \backslash$ Version=DEC-AXP-OSF $/ 1-$ G9 8RevA $.11 .3 \backslash \mathrm{HF}=-11$ $45.3573094 \backslash \mathrm{RMSD}=5.445 \mathrm{e}-09 \backslash \mathrm{RMSF}=1.591 \mathrm{e}-05 \backslash \mathrm{Dipole}=-0.9605393,0.0492055,0$ $.0284694 \backslash \mathrm{PG}=\mathrm{C} 01 \quad[\mathrm{X}(\mathrm{C} 9 \mathrm{H} 10 \mathrm{~S} 2)] \backslash \backslash \mathrm{Q}$

\section{$\mathrm{S}=\mathrm{C}\left(\mathrm{CH}_{3}\right) \mathrm{S}-\mathrm{CH}(\mathrm{Ph}) \mathrm{CH}_{3}$}

$1 \backslash 1 \backslash G I N C-S C 29 \backslash F O p t \backslash R B 3 L Y P \backslash 6-31 G(d) \backslash C 10 H 12 S 2 \backslash M L C 501 \backslash 02-J u 1-2004 \backslash 0 \backslash \backslash \#$ B3

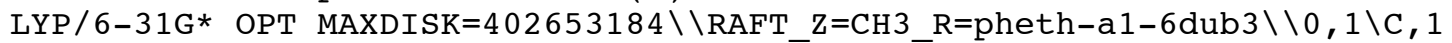
$.8274382095,-0.249707204,-3.4578286492 \backslash \mathrm{C}, 1.7 \overline{9} 17778162,-0.2747141709,-1$ $.9424462533 \backslash \mathrm{S}, 3.1664685743,-0.2797568442,-1.032255372 \backslash \mathrm{S}, 0.1373911524,-$ $0.3164708104,-1.3573337192 \backslash \mathrm{C}, 0.2728618406,-0.4524373246,0.4952594765 \backslash \mathrm{H}$ $, 1.1611040652,0.136770583,0.7434983761 \backslash \mathrm{C}, 0.5028798889,-1.9060119556,0$. $9232986023 \backslash \mathrm{C},-0.9574496829,0.1939021194,1.1006126398 \backslash \mathrm{C},-0.8458325629,1$ $.4287593708,1.7531850757 \backslash \mathrm{C},-2.2185439336,-0.4158394684,1.0295205741 \backslash \mathrm{H}$, $0.1250445823,1.9149526317,1.8131121953 \backslash \mathrm{H},-2.3302261248,-1.3716936081,0$ $.5245851886 \backslash \mathrm{C},-1.9619211313,2.0368780464,2.3284292459 \backslash \mathrm{C},-3.3369021244$, $0.1931287125,1.5987095931 \backslash \mathrm{H},-1.8541576512,2.9921440027,2.8351987099 \backslash \mathrm{H}$, $-4.3067504973,-0.2927080655,1.5324689698 \backslash \mathrm{C},-3.2117326284,1.4205218081$, $2.2521264584 \backslash \mathrm{H},-4.0825524227,1.893010618,2.6984358184 \backslash \mathrm{H}, 1.4253339621$, $2.2919967447,0.4812660697 \backslash \mathrm{H},-0.3263638869,-2.5547998566,0.6257607256 \backslash \mathrm{H}$ , $0.5985006455,-1.9519201973,2.0144714623 \backslash \mathrm{H}, 2.6457062085,0.3892935096,-$ $3.7983705421 \backslash \mathrm{H}, 0.8849625937,0.094603437,-3.8972091512 \backslash \mathrm{H}, 2.0221887496,-$ $1.2648934371,-3.8249929431 \backslash \backslash$ Version=DEC-AXP-OSF / 1-G03RevB . 03 \State=1-A $\backslash \mathrm{HF}=-1184.6701658 \backslash \mathrm{RMSD}=5.512 \mathrm{e}-09 \backslash \mathrm{RMSF}=1.322 \mathrm{e}-06 \backslash \mathrm{Dipole}=-0.9119129,-0.0$ $367753,0.0409486 \backslash \mathrm{PG}=\mathrm{C} 01 \quad[\mathrm{X}(\mathrm{C} 10 \mathrm{H} 12 \mathrm{~S} 2)] \backslash \backslash @$ 


\section{$\mathrm{S}=\mathrm{C}\left(\mathrm{CH}_{3}\right) \mathrm{S}-\mathrm{C}(\mathrm{Ph})\left(\mathrm{CH}_{3}\right)_{2}$}

$1 \backslash 1 \backslash G I N C-S C 9 \backslash F O p t \backslash R B 3 L Y P \backslash 6-31 G(d) \backslash C 11 H 14 S 2 \backslash M L C 501 \backslash 02-J u 1-2004 \backslash 0 \backslash \backslash \#$ B3L YP /6-31G* OPT MAXDISK $=402653184 \backslash \backslash$ RAFT_Z=CH3_R=cumyl-a1-6dub3 $\backslash \backslash 0,1 \backslash \mathrm{C}, 2$. $554810971,0.0628373755,0.8708730422 \backslash \mathrm{S}, 2.532 \overline{1} 430928,0.0222117331,2.5201$ $613305 \backslash \mathrm{C}, 3.8709954546,0.084378493,0.1097040788 \backslash \mathrm{S}, 1.2273435686,0.051885$ $898,-0.2775877982 \backslash \mathrm{C},-0.4648646262,-0.0951025635,0.5756625861 \backslash \mathrm{C},-0.5222$ $140475,-1.4125977057,1.3582759289 \backslash \mathrm{C},-0.7135138848,1.0988791502,1.51520$ $68975 \backslash C,-1.4293078299,-0.0503419923,-0.6198999832 \backslash C,-2.2730699383,-1.1$ $241705446,-0.9362111961 \backslash \mathrm{C},-1.5011780694,1.1002980874,-1.4247790797 \backslash \mathrm{H},-$ $2.2489200588,-2.0319935767,-0.3446168338 \backslash \mathrm{H},-0.8512079128,1.9451864879$, $-1.2169275859 \backslash \mathrm{C},-3.1654246666,-1.046997441,-2.0080151514 \backslash \mathrm{C},-2.38599910$ $55,1.1770144048,-2.4982322564 \backslash \mathrm{H},-3.8117990476,-1.8929393824,-2.2264719$ $379 \backslash \mathrm{H},-2.4180604441,2.0787848291,-3.1038290052 \backslash \mathrm{C},-3.2263217365,0.10217$ $75667,-2.7939182692 \backslash \mathrm{H},-3.9190648903,0.1605045996,-3.629021903 \backslash \mathrm{H},-0.005$ $4041505,1.0943987361,2.3460106326 \backslash \mathrm{H}, 0.2089153908,-1.3995238871,2.16839$ $19651 \backslash \mathrm{H},-1.5176263205,-1.5339730513,1.8025819389 \backslash \mathrm{H},-1.729849172,1.0175$ $164062,1.9198353558 \backslash \mathrm{H},-0.3177118747,-2.2768025657,0.7199945394 \backslash \mathrm{H},-0.63$ $52241075,2.0556541957,0.9931375354 \backslash \mathrm{H}, 4.5866388969,0.7219259673,0.63449$ $36944 \backslash \mathrm{H}, 4.2823033939,-0.9320691716,0.0871732224 \backslash \mathrm{H}, 3.7617485886,0.42951$ $93325,-0.9239277201 \backslash \backslash$ Version=DEC-AXP-OSF $/ 1-G 03$ RevB . 03 \State $=1-A \backslash H F=-12$ $23.9747021 \backslash \mathrm{RMSD}=6.846 \mathrm{e}-09 \backslash \mathrm{RMSF}=2.778 \mathrm{e}-06 \backslash \mathrm{Dipole}=-0.6225142,-0.0340942$, $-0.8279907 \backslash \mathrm{PG}=\mathrm{C} 01 \quad[\mathrm{X}(\mathrm{C} 11 \mathrm{H} 14 \mathrm{~S} 2)] \backslash \backslash @$

\section{Table S3. Geometries of the RAFT-adduct Radicals $\left(\mathrm{CH}_{3} \mathrm{SC} \bullet(\mathrm{Z}) \mathrm{S}-\mathrm{CH}_{3}\right)$, and Thiocarbonyl Products $\left(\mathrm{CH}_{3} \mathrm{SC}(\mathrm{Z})=\mathrm{S}\right)$ of Beta-Scission, Used in Calculating the Beta-Scission Enthalpies in Table 3}

\section{$\mathrm{CH}_{3} \mathrm{SC} \cdot\left(\mathrm{CH}_{3}\right) \mathrm{S}-\mathrm{CH}_{3}$}

$1 \backslash 1 \backslash G I N C-S C 65 \backslash F O p t \backslash U B 3 L Y P \backslash 6-31 G(d) \backslash C 4 H 9 S 2(2) \backslash M L C 501 \backslash 16-J u 1-2002 \backslash 1 \backslash \backslash \# N$ UB3LYP/6-31G(D) FOPT $=(Z-M A T R I X$, TIGHT, MAXCYC=100) FREQ MAXDISK $=13107200$ $0 \mathrm{SCF}=(\mathrm{TIGHT}, \mathrm{MAXCYC}=100)$ GEOM=CHECK GUESS $=$ READ $\backslash \backslash$ mescmesme $-\mathrm{C} 1 \mathrm{~b}-6 \mathrm{dub} 3 \backslash \backslash 0$ , $2 \backslash \mathrm{H} \backslash \mathrm{C}, 1, \mathrm{~B} 1 \backslash \mathrm{H}, 2, \mathrm{~B} 2,1, \mathrm{~A} 1 \backslash \mathrm{S}, 2, \mathrm{~B} 3,1, \mathrm{~A} 2,3, \mathrm{D} 1,0 \backslash \mathrm{C}, 4, \mathrm{~B} 4,2, \mathrm{~A} 3,1, \mathrm{D} 2,0 \backslash \mathrm{S}, 5, \mathrm{~B} 5,4$ , A4 , 2, D3 , $0 \backslash \mathrm{C}, 6, \mathrm{~B} 6,5, \mathrm{~A} 5,4,-\mathrm{D} 4,0 \backslash \mathrm{H}, 7, \mathrm{~B} 7,6, \mathrm{~A} 6,5, \mathrm{D} 5,0 \backslash \mathrm{H}, 7, \mathrm{~B} 8,6, \mathrm{~A} 7,8, \mathrm{D} 6,0 \backslash \mathrm{H}$ $, 7, \mathrm{~B} 9,6, \mathrm{~A} 8,8, \mathrm{D} 7,0 \backslash \mathrm{C}, 5, \mathrm{~B} 10,4, \mathrm{~A} 9,2$, $\mathrm{D} 8,0 \backslash \mathrm{H}, 2$, $\mathrm{B} 11,1, \mathrm{~A} 10,3, \mathrm{D} 9,0 \backslash \mathrm{H}, 11, \mathrm{~B} 12,5$, $\mathrm{A} 11,4, \mathrm{D} 10,0 \backslash \mathrm{H}, 11, \mathrm{~B} 13,5, \mathrm{~A} 12,4, \mathrm{D} 11,0 \backslash \mathrm{H}, 11, \mathrm{~B} 14,5, \mathrm{~A} 13,4, \mathrm{D} 12,0 \backslash \backslash \mathrm{B} 1=1.092657$ $93 \backslash B 2=1.09285939 \backslash B 3=1.83510395 \backslash B 4=1.77013049 \backslash B 5=1.75804982 \backslash B 6=1.832220$ $08 \backslash B 7=1.09257315 \backslash B 8=1.09085787 \backslash B 9=1.09328827 \backslash B 10=1.50447696 \backslash B 11=1.0934$ $6331 \backslash \mathrm{B} 12=1.09613505 \backslash \mathrm{B} 13=1.09553397 \backslash \mathrm{B} 14=1.10178689 \backslash \mathrm{A} 1=110.0297286 \backslash \mathrm{A} 2=11$ $0.82443615 \backslash \mathrm{A} 3=102.23773298 \backslash \mathrm{A} 4=118.51568336 \backslash \mathrm{A} 5=103.96848806 \backslash \mathrm{A} 6=110.4950$ $1693 \backslash A 7=111.21266523 \backslash \mathrm{A} 8=105.91951214 \backslash \mathrm{A} 9=120.43443778 \backslash \mathrm{A} 10=109.33705389 \backslash$ $\mathrm{A} 11=111.15729093 \backslash \mathrm{A} 12=110.74166016 \backslash \mathrm{A} 13=112.20742397 \backslash \mathrm{D} 1=-124.06148497 \backslash \mathrm{D} 2$ $=60.56470818 \backslash D 3=-144.49451711 \backslash D 4=-48.977946 \backslash D 5=60.52936234 \backslash D 6=-122.478$ $49479 \backslash D 7=118.55770463 \backslash D 8=62.40591497 \backslash D 9=119.28641302 \backslash D 10=-44.11186517 \backslash$ D1 $1=-164.00422423 \backslash D 12=75.46393503 \backslash \backslash$ Version=DEC-AXP-OSF / 1-G98RevA.11.3 $\mathrm{HF}=-954.1760596 \backslash \mathrm{S} 2=0.755169 \backslash \mathrm{S} 2-1=0 . \backslash \mathrm{S} 2 \mathrm{~A}=0.75002 \backslash \mathrm{RMSD}=5.132 \mathrm{e}-09 \backslash \mathrm{RMSF}=2$. $815 \mathrm{e}-05 \backslash \mathrm{Dipole}=0.2098476,0.5512682,-0.4657697 \backslash \mathrm{PG}=\mathrm{C} 01 \quad[\mathrm{X}(\mathrm{C} 4 \mathrm{H} 9 \mathrm{~S} 2)] \backslash \backslash @$

\section{$\mathrm{S}=\mathrm{C}\left(\mathrm{CH}_{3}\right) \mathrm{SCH}_{3}$}

$1 \backslash 1 \backslash G I N C-S C 100 \backslash F O p t \backslash R B 3 L Y P \backslash 6-31 G(d) \backslash C 3 H 6 S 2 \backslash M L C 501 \backslash 17-J u l-2002 \backslash 1 \backslash \backslash \# P$ RB $3 L Y P / 6-31 \mathrm{G} *$ FOPT $=($ MAXCYC $=150, \mathrm{Z}-$ MATRIX $)$ MAXDISK $=131072000$ FREQ SCF $=$ TIGH T GEOM=CHECK GUESS=READ $\backslash \backslash \mathrm{S}=\mathrm{C}(\mathrm{Me})-\mathrm{S}-\mathrm{Me} \mathrm{C} 1 \backslash \backslash 0,1 \backslash \mathrm{S} \backslash \mathrm{C}, 1, \mathrm{~b} 1 \backslash \mathrm{C}, 2, \mathrm{~b} 2,1, \mathrm{a} 1 \backslash \mathrm{S}, 2$ , b3 , 1, a2 , 3, dh $3,0 \backslash \mathrm{C}, 4, \mathrm{~b} 4,2, \mathrm{a} 3,1, \mathrm{dh} 4,0 \backslash \mathrm{H}, 5, \mathrm{~b} 5,4, \mathrm{a} 4,2, \mathrm{dh} 5,0 \backslash \mathrm{H}, 5, \mathrm{~b} 6,4, \mathrm{a} 5,2$ , dh $1,0 \backslash \mathrm{H}, 5, \mathrm{~b} 6 \mathrm{a}, 4, \mathrm{a} 5 \mathrm{a}, 2,-\mathrm{dh} 1 \mathrm{a}, 0 \backslash \mathrm{H}, 3, \mathrm{~b} 7,2, \mathrm{a} 6,1, \mathrm{dh} 6,0 \backslash \mathrm{H}, 3, \mathrm{~b} 8,2, \mathrm{a} 7,1, \mathrm{dh} 2,0$ $\backslash \mathrm{H}, 3, \mathrm{~b} 8 \mathrm{a}, 2, \mathrm{a} 7 \mathrm{a}, 1,-\mathrm{dh} 2 \mathrm{a}, 0 \backslash \backslash \mathrm{b} 1=1.64599636 \backslash \mathrm{b} 2=1.5148461 \backslash \mathrm{b} 3=1.75511783 \backslash \mathrm{b} 4=$ $1.81794969 \backslash \mathrm{b} 5=1.09307671 \backslash \mathrm{b} 6=1.0919738 \backslash \mathrm{b} 6 \mathrm{a}=1.09197036 \backslash \mathrm{b} 7=1.09324611 \backslash \mathrm{b} 8=$ $1.09537804 \backslash b 8 a=1.0962657 \backslash a 1=122.84648556 \backslash a 2=125$. $75342086 \backslash a 3=103.776055$ 
$3 \backslash a 4=106.19073532 \backslash a 5=110.33849971 \backslash a 5 a=110.27255399 \backslash a 6=109 \cdot 69980988 \backslash a 7=$ $112.81878244 \backslash a 7 a=109.03573022 \backslash d h 1=60.70823437 \backslash d h 1 a=60.13720525 \backslash d h 2=167$ $.56947484 \backslash \mathrm{dh} 2 \mathrm{a}=72.03339216 \backslash \mathrm{dh} 3=181.23401229 \backslash \mathrm{dh} 4=0.90237034 \backslash \mathrm{dh} 5=180.305$ $77272 \backslash \mathrm{dh} 6=45.4338183 \backslash \backslash$ Version=DEC-AXP-OSF $/ 1-G 98 \mathrm{RevA} .11 .3 \backslash \mathrm{HF}=-914.30737$ $69 \backslash \mathrm{RMSD}=3.465 \mathrm{e}-10 \backslash \mathrm{RMSF}=1.676 \mathrm{e}-06 \backslash \mathrm{Dipole}=-0.3544958,-0.0417535,0.694415$ $9 \backslash \mathrm{PG}=\mathrm{C} 01 \quad[\mathrm{X}(\mathrm{C} 3 \mathrm{H} 6 \mathrm{~S} 2)] \backslash \backslash @$

\section{$\mathrm{CH}_{3} \mathrm{SC} \cdot(\mathrm{H}) \mathrm{S}-\mathrm{CH}_{3}$}

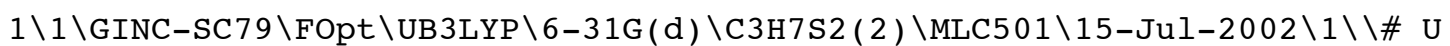
B3LYP /6-31G* FOPT $=(Z-M A T R I X, M A X C Y C=100)$ FREQ MAXDISK $=131072000$ SCF $=(\mathrm{TI}$ GHT , MAXCYC $=100)$ GEOM=CHECK $\backslash \backslash$ meschsme-c $1 b-6 d u b 3 \backslash \backslash 0,2 \backslash H \backslash C, 1, B 1 \backslash \mathrm{H}, 2, \mathrm{~B} 2,1$, $\mathrm{A} 1 \backslash \mathrm{S}, 2, \mathrm{~B} 3,1, \mathrm{~A} 2,3, \mathrm{D} 1,0 \backslash \mathrm{C}, 4, \mathrm{~B} 4,2, \mathrm{~A} 3,1, \mathrm{D} 2,0 \backslash \mathrm{S}, 5, \mathrm{~B} 5,4, \mathrm{~A} 4,2, \mathrm{D} 3,0 \backslash \mathrm{C}, 6, \mathrm{~B} 6,5, \mathrm{~A}$ $5,4,-\mathrm{D} 4,0 \backslash \mathrm{H}, 7, \mathrm{~B} 7,6, \mathrm{~A} 6,5, \mathrm{D} 5,0 \backslash \mathrm{H}, 7, \mathrm{~B} 8,6, \mathrm{~A} 7,8, \mathrm{D} 6,0 \backslash \mathrm{H}, 7, \mathrm{~B} 9,6, \mathrm{~A} 8,8, \mathrm{D} 7,0 \backslash \mathrm{H}, 5$ $, \mathrm{B} 10,4, \mathrm{~A} 9,2, \mathrm{D} 8,0 \backslash \mathrm{H}, 2, \mathrm{~B} 11,1, \mathrm{~A} 10,3, \mathrm{D} 9,0 \backslash \backslash \mathrm{B} 1=1.09245285 \backslash \mathrm{B} 2=1.0938637 \backslash \mathrm{B} 3=1$ $.83125984 \backslash \mathrm{B} 4=1.75500785 \backslash \mathrm{B} 5=1.74076721 \backslash \mathrm{B} 6=1.83589742 \backslash \mathrm{B} 7=1.09250381 \backslash \mathrm{B} 8=1$ $.09126763 \backslash B 9=1.09314848 \backslash B 10=1.08750952 \backslash B 11=1.09312093 \backslash A 1=109.89583381 \backslash$ $\mathrm{A} 2=110.82435935 \backslash \mathrm{A} 3=100.23333582 \backslash \mathrm{A} 4=122.50571955 \backslash \mathrm{A} 5=103.40041602 \backslash \mathrm{A} 6=110$ $.32952264 \backslash A 7=111.31841879 \backslash A 8=106.03792498 \backslash A 9=118.05982812 \backslash A 10=109.5874$ $6805 \backslash D 1=-122.6210925 \backslash D 2=64.69226164 \backslash D 3=-158.0946175 \backslash D 4=-51.30757768 \backslash D 5$ $=59.46321196 \backslash \mathrm{D} 6=-122.46199608 \backslash \mathrm{D} 7=118.68665702 \backslash \mathrm{D} 8=47.35009589 \backslash \mathrm{D} 9=119.69$ $751863 \backslash \backslash$ Version=DEC-AXP-OSF $/ 1-G 98 R$ RevA. $11.3 \backslash \mathrm{HF}=-914.8590203 \backslash \mathrm{S} 2=0.755128$ $\backslash \mathrm{S} 2-1=0 . \backslash \mathrm{S} 2 \mathrm{~A}=0.75002 \backslash \mathrm{RMSD}=5.785 \mathrm{e}-09 \backslash \mathrm{RMSF}=1.037 \mathrm{e}-05 \backslash \mathrm{Dipole}=0.0181274,0$. $5574054,-0.4697362 \backslash \mathrm{PG}=\mathrm{C} 01 \quad[\mathrm{X}(\mathrm{C} 3 \mathrm{H} 7 \mathrm{~S} 2)] \backslash \backslash @$

\section{$\mathrm{S}=\mathrm{C}(\mathrm{H}) \mathrm{SCH}_{3}$}

$1 \backslash 1 \backslash G I N C-S C 28 \backslash F O p t \backslash R B 3 L Y P \backslash 6-31 G(d) \backslash C 2 H 4 S 2 \backslash M L C 501 \backslash 16-J u 1-2002 \backslash 1 \backslash \backslash \# P$ RB3 LYP $/ 6-31 G *$ FOPT $=($ TIGHT, MAXCYC $=150$, Z-MATRIX $)$ MAXDISK $=131072000$ FREQ SCF $=\mathrm{TIGHT} \backslash \backslash \mathrm{S}=\mathrm{C}(\mathrm{H})-\mathrm{S}-\mathrm{Me} \mathrm{Cs}(2 \mathrm{a}) \backslash \backslash 0,1 \backslash \mathrm{S} \backslash \mathrm{C}, 1, \mathrm{~b} 1 \backslash \mathrm{H}, 2, \mathrm{~b} 2,1, \mathrm{a} 1 \backslash \mathrm{S}, 2, \mathrm{~b} 3,1, \mathrm{a} 2,3,18$ 0 . , $0 \backslash \mathrm{C}, 4, \mathrm{~b} 4,2, \mathrm{a} 3,1,0 ., 0 \backslash \mathrm{H}, 5, \mathrm{~b} 5,4, \mathrm{a} 4,2,180,0 \backslash \mathrm{H}, 5, \mathrm{~b} 6,4, \mathrm{a} 5,2, \mathrm{dh} 1,0 \backslash \mathrm{H}, 5, \mathrm{~b}$ $6,4, a 5,2,-d h 1,0 \backslash \backslash \mathrm{b} 1=1.63746361 \backslash \mathrm{b} 2=1.09330009 \backslash \mathrm{b} 3=1.73755944 \backslash \mathrm{b} 4=1.820696$ $57 \backslash \mathrm{b} 5=1.09233155 \backslash \mathrm{b} 6=1.09203481 \backslash \mathrm{a} 1=121.28809715 \backslash \mathrm{a} 2=129.63000423 \backslash \mathrm{a} 3=103$. $10279807 \backslash \mathrm{a} 4=106.35470404 \backslash \mathrm{a} 5=110.18674431 \backslash \mathrm{dh} 1=60.3841272 \backslash \backslash$ Version=DEC $-\mathrm{A}$ $\mathrm{XP}-\mathrm{OSF} / 1-\mathrm{G} 98 \mathrm{RevA} .11 .3 \backslash$ State $=1-\mathrm{A}^{\prime} \backslash \mathrm{HF}=-874.9871381 \backslash \mathrm{RMSD}=5.554 \mathrm{e}-09 \backslash \mathrm{RMSF}=5$ $.472 \mathrm{e}-06 \backslash \mathrm{Dipole}=-0.6513287,0 ., 0.6109301 \backslash \mathrm{PG}=\mathrm{CS}[\mathrm{SG}(\mathrm{C} 2 \mathrm{H} 2 \mathrm{~S} 2), \mathrm{X}(\mathrm{H} 2)] \backslash \backslash @$

\section{$\mathrm{CH}_{3} \mathrm{SC} \cdot(\mathrm{F}) \mathrm{SCH}_{3}$}

$1 \backslash 1 \backslash$ GINC-SC57 \FOpt \UB3LYP \6-31G(d) \C3H6F1S2 ( 2$) \backslash M L C 501 \backslash 25-M a y-2004 \backslash 0 \backslash \backslash \#$ B3LYP/6-31G* OPT FREQ MAXDISK=268435456\\rrad $z=f \quad r=m e-c 1 a-6 d u b 3 \backslash \backslash 0,2$ $\backslash \mathrm{H},-1.4608094972,-0.0709694822,2.7866654428 \backslash \mathrm{C},-0.3 \overline{7} 01360144,-0.0132859$ $973,2.7604246302 \backslash \mathrm{H},-0.047144627,1.0287818623,2.7724556354 \backslash \mathrm{S}, 0.28238046$ $58,-0.8622651787,1.2714810842 \backslash \mathrm{C},-0.3854096711,0.1615973635,0.012289989$ $3 \backslash S,-0.4256627666,-0.3584314418,-1.6511070005 \backslash \mathrm{C}, 1.0680954105,0.4678850$ $636,-2.3567223078 \backslash \mathrm{H}, 1.9823767235,-0.0023678665,-1.9875450804 \backslash \mathrm{H}, 1.05540$ $14148,1.5286802503,-2.1012022026 \backslash \mathrm{H}, 1.0046500744,0.3524651747,-3.441622$ $0922 \backslash \mathrm{F},-0.240367635,1.5038191257,0.2138987402 \backslash \mathrm{H}, 0.0460530895,-0.536994$ $7221,3.6242204267 \backslash \backslash$ Version=DEC-AXP-OSF $/ 1-G 03$ RevB. 03 $\backslash$ State $=2-A \backslash H F=-1014$ $.0864778 \backslash \mathrm{S} 2=0.755211 \backslash \mathrm{S} 2-1=0 . \backslash \mathrm{S} 2 \mathrm{~A}=0.750019 \backslash \mathrm{RMSD}=5.746 \mathrm{e}-09 \backslash \mathrm{RMSF}=8.505 e-0$ $6 \backslash \mathrm{Dipole}=0.3608014,0.2695271,0.2839685 \backslash \mathrm{PG}=\mathrm{C} 01 \quad[\mathrm{X}(\mathrm{C} 3 \mathrm{H} 6 \mathrm{~F} 1 \mathrm{~S} 2)] \backslash \backslash @$

\section{$\mathrm{S}=\mathrm{C}(\mathrm{F}) \mathrm{SCH}_{3}$}

$1 \backslash 1 \backslash G I N C-S C 26 \backslash F O p t \backslash R B 3 L Y P \backslash 6-31 G(d) \backslash C 2 H 3 F 1 S 2 \backslash M L C 501 \backslash 25-M a y-2004 \backslash 0 \backslash \backslash \#$ B3 LYP/6-31G* OPT FREQ SCF=TIGHT MAXDISK=402653184 \raft_z=f_r=me-c1-6dub $3 \backslash \backslash 0,1 \backslash S, 0.6732495754,0 .,-1.5951188068 \backslash C, 0.6539442516,0 ., \overline{0} .0289884936 \backslash$ $\mathrm{F}, 1.785438625,0.0 .7564633643 \backslash \mathrm{S},-0.6766528731,0 ., 1.1663944045 \backslash \mathrm{C},-2.118$ $3749953,0 ., 0.0535320972 \backslash \mathrm{H},-2.9969946804,0.0 .7038462211 \backslash \mathrm{H},-2.115457859$ $8,-0.8932702421,-0.5737748037 \backslash \mathrm{H},-2.1154578598,0.8932702421,-0.57377480$ $37 \backslash \backslash$ Version=DEC-AXP-OSF $/ 1-G 03$ RevB . 03 \State $=1-A^{\prime} \backslash H F=-974.2219532 \backslash \mathrm{RMSD}=5$ 
$.980 \mathrm{e}-09 \backslash \mathrm{RMSF}=3.562 \mathrm{e}-05 \backslash \mathrm{Dipole}=-1.0976782,0 ., 0.3076847 \backslash \mathrm{PG}=\mathrm{CS} \quad[\mathrm{SG}(\mathrm{C} 2 \mathrm{H} 1 \mathrm{~F}$ $1 \mathrm{~S} 2), \mathrm{X}(\mathrm{H} 2) \mathrm{]} \backslash \backslash @$

\section{$\mathrm{CH}_{3} \mathrm{SC} \cdot(\mathrm{Cl}) \mathrm{SCH}_{3}$}

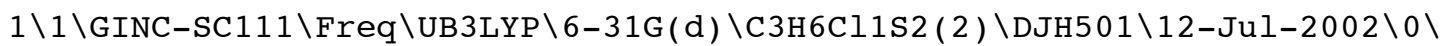
$\backslash \# \mathrm{~N}$ UB3LYP/6-31G(D) SCF=TIGHT FREQ MAXDISK=39321600\\CH3SCClSCH3 radic al UB3LYP / 6-31G(d) freq $\backslash \backslash 0,2 \backslash \mathrm{Cl}, 0 ., 0 ., 1.7458857534 \backslash \mathrm{C}, 0 \ldots, 0 .,-0.015644$ $2466 \backslash \mathrm{S}, 0.730536,1.315209,-0.8890652466 \backslash \mathrm{S},-0.730536,-1.315209,-0.889065$ $2466 \backslash \mathrm{C}, 0 ., 2.808624,-0.1091052466 \backslash \mathrm{C}, 0 \ldots,-2.808624,-0.1091052466 \backslash \mathrm{H},-1.080$ $263,2.837085,-0.2685442466 \backslash \mathrm{H}, 0.227201,2.837952,0.9583197534 \backslash \mathrm{H}, 0.468179$ $, 3.664216,-0.6031962466 \backslash \mathrm{H}, 1.080263,-2.837085,-0.2685442466 \backslash \mathrm{H},-0.227201$ $,-2.837952,0.9583197534 \backslash \mathrm{H},-0.468179,-3.664216,-0.6031962466 \backslash \backslash$ Version=D EC-AXP-OSF / 1-G98RevA. 11.3 SState $=2-B \backslash H F=-1374.454289 \backslash \mathrm{S} 2=0.755828 \backslash \mathrm{S} 2-1=0$ $. \backslash \mathrm{S} 2 \mathrm{~A}=0.750025 \backslash \mathrm{RMSD}=5.641 \mathrm{e}-09 \backslash \mathrm{RMSF}=1.672 \mathrm{e}-06 \backslash \mathrm{Dipole}=0,0 ., 0.1376501 \backslash$ $\mathrm{PG}=\mathrm{C} 02 \quad[\mathrm{C} 2(\mathrm{C} 1 \mathrm{Cl} 1), \mathrm{X}(\mathrm{C} 2 \mathrm{H} 6 \mathrm{~S} 2)] \backslash \backslash @$

\section{$\mathrm{CH}_{3} \mathrm{~S}(\mathrm{Cl}) \mathrm{C}=\mathrm{S}$}

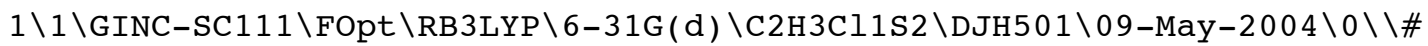
B3LYP/6-31G* SCF=TIGHT OPT=TIGHT MAXDISK=39321600 \CH3SCCl=Sa B3LYP / $6-31 \mathrm{G}(\mathrm{d})$ geom opt $\backslash \backslash 0,1 \backslash \mathrm{Cl}, 0.033089777,0,-2.0304760456 \backslash \mathrm{C},-0.2403988803$ , 0 . $,-0.2710280846 \backslash S, 1.3166697464,0 \ldots, 0.5239390117 \backslash \mathrm{S},-1.7280471353,0$. 0 . $3846713207 \backslash \mathrm{C}, 0.8528974077,0 ., 2.2853874397 \backslash \mathrm{H}, 1.7963221294,0 ., 2.83822126$ $17 \backslash \mathrm{H}, 0.2740993601,0.8932837385,2.527975033 \backslash \mathrm{H}, 0.2740993601,-0.893283738$ $5,2.527975033 \backslash \backslash$ Version=DEC-AXP-OSF / 1-G03RevB . 05 \State=1-A $\backslash \mathrm{HF}=-1334.58$ $1472 \backslash \mathrm{RMSD}=2.831 \mathrm{e}-09 \backslash \mathrm{RMSF}=1.027 \mathrm{e}-06 \backslash \mathrm{Dipole}=0.6442117,0.0 .9198297 \backslash \mathrm{PG}=\mathrm{CS}$ $[\mathrm{SG}(\mathrm{C} 2 \mathrm{H} 1 \mathrm{Cl} 1 \mathrm{~S} 2), \mathrm{X}(\mathrm{H} 2)] \backslash \backslash @$

\section{$\mathrm{CH}_{3} \mathrm{SC} \cdot(\mathrm{CN}) \mathrm{SCH}_{3}$}

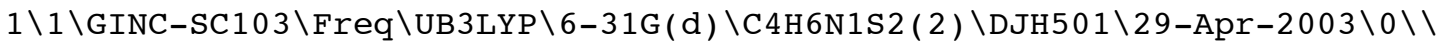
\#N UB3LYP/6-31G(D) SCF=TIGHT FREQ MAXDISK=39321600\\CH3SC(CN)SCH3d rad

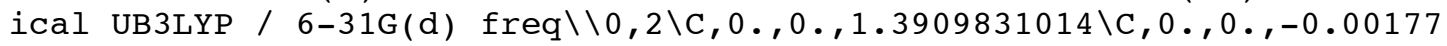
$88986 \backslash \mathrm{S}, 0.1 .48737,-0.9364158986 \backslash \mathrm{C},-0.95775,2.619045,0.1386721014 \backslash \mathrm{S}, 0$. $,-1.48737,-0.9364158986 \backslash \mathrm{C}, 0.95775,-2.619045,0.1386721014 \backslash \mathrm{H},-1.992213,2$ $.280518,0.2285301014 \backslash \mathrm{H},-0.499302,2.697444,1.1267991014 \backslash \mathrm{H},-0.93062,3.59$ $448,-0.3533868986 \backslash \mathrm{H}, 1.992213,-2.280518,0.2285301014 \backslash \mathrm{H}, 0.499302,-2.6974$ $44,1.1267991014 \backslash \mathrm{H}, 0.93062,-3.59448,-0.3533868986 \backslash \mathrm{N}, 0 ., 0 ., 2.5660191014 \backslash$ \Version=DEC-AXP-OSF $/ 1-G 98 R e v A .11 .3 \backslash$ State $=2-B \backslash H F=-1007.1120674 \backslash S 2=0.75$ $9884 \backslash \mathrm{S} 2-1=0 . \backslash \mathrm{S} 2 \mathrm{~A}=0.750062 \backslash \mathrm{RMSD}=6.107 \mathrm{e}-09 \backslash \mathrm{RMSF}=4.470 \mathrm{e}-07 \backslash \mathrm{Dipole}=0 ., 0 .,-$ $0.6530448 \backslash \mathrm{PG}=\mathrm{C} 02 \quad[\mathrm{C} 2(\mathrm{C} 1 \mathrm{C} 1 \mathrm{~N} 1), \mathrm{X}(\mathrm{C} 2 \mathrm{H} 6 \mathrm{~S} 2)] \backslash \backslash @$

\section{$\mathrm{CH}_{3} \mathrm{~S}(\mathrm{CN}) \mathrm{C}=\mathrm{S}$}

$1 \backslash 1 \backslash G I N C-S C 126 \backslash F O p t \backslash R B 3 L Y P \backslash 6-31 G(d) \backslash C 3 H 3 N 1 S 2 \backslash D J H 501 \backslash 10-M a y-2004 \backslash 0 \backslash \backslash \# B$ 3LYP/6-31G* SCF=TIGHT OPT=TIGHT MAXDISK=39321600 IOP $(1 / 8=10) \backslash \backslash \mathrm{CH} 3 \mathrm{SC}(\mathrm{CN}$

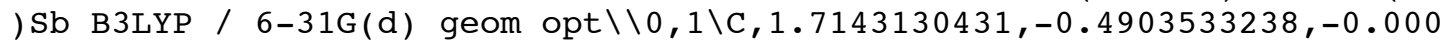
$0026937 \backslash \mathrm{C}, 0.405329302,0.1041091147,-0.0000016782 \backslash \mathrm{S},-0.8432374325,-1.12$ $90645757,0.0000193385 \backslash \mathrm{C},-2.3647846875,-0.1341256382,0.0000040713 \backslash \mathrm{S}, 0.2$ $21723625,1.7406005545,-0.0000181688 \backslash \mathrm{H},-3.1883355575,-0.8523386291,-0.0$ $000190697 \backslash \mathrm{H},-2.4091215943,0.4936780571,0.8925537675 \backslash \mathrm{H},-2.4090883069,0$. $4936981649,-0.8925330863 \backslash \mathrm{N}, 2.7745172047,-0.9710563101,-0.0000026461 \backslash \backslash \mathrm{V}$ ersion=DEC-AXP-OSF /1-G03RevB.05 \State=1-A \HF=-967.2190127 $\backslash$ RMSD=7 . 862e$09 \backslash \mathrm{RMSF}=2.473 e-06 \backslash \mathrm{Dipole}=-1.8143545,-0.0479982,0.0000052 \backslash \mathrm{PG}=\mathrm{C} 01][\mathrm{X}(\mathrm{C} 3 \mathrm{H}$ $3 N 1 S 2$ ) ] \\

\section{$\mathrm{CH}_{3} \mathrm{SC} \cdot\left(\mathrm{CF}_{3}\right) \mathrm{SCH}_{3}$}

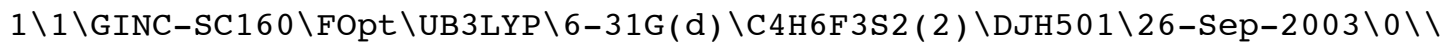
\#N UB3LYP/6-31G(D) SCF=TIGHT OPT=CALCFC MAXDISK=39321600\\CH3SC(CF3)SC 
H3 radical UB3LYP / 6-31G(d) geom opt \\0,2\F,-1.0471802293,1.525585842 $6,-1.015434 \backslash \mathrm{C},-0.2091711696,1.1281099686,-0.017138 \backslash \mathrm{C}, 0.10325005,-0.332$ $4039845,-0.076694 \backslash S, 1.6484101431,-0.9523747522,-0.608042 \backslash \mathrm{C}, 2.835255051$ $4,-0.3419965739,0.660132 \backslash \mathrm{S},-1.1071517692,-1.5357321664,0.274576 \backslash \mathrm{C},-2.7$ $2002889,-0.7319804088,-0.070076 \backslash \mathrm{H},-2.7932929324,-0.4497534198,-1.12146$ $6 \backslash \mathrm{H},-2.8675600212,0.140908569,0.566798 \backslash \mathrm{F}, 0.8965477154,1.8936931348,-0$. $119665 \backslash \mathrm{F},-0.8305872208,1.4690158752,1.142202 \backslash \mathrm{H}, 2.5690871098,-0.7306406$ $139,1.64556 \backslash \mathrm{H}, 2.8669508877,0.7474174309,0.673303 \backslash \mathrm{H}, 3.8124831104,-0.734$ $612427,0.365461 \backslash \mathrm{H},-3.4726507763,-1.4886305219,0.164543 \backslash \backslash$ Version=DEC $-\mathrm{AX}$ $\mathrm{P}-\mathrm{OSF} / 1-\mathrm{G} 03 \mathrm{RevB} .02 \backslash \mathrm{State}=2-\mathrm{A} \backslash \mathrm{HF}=-1251.8950979 \backslash \mathrm{S} 2=0.754673 \backslash \mathrm{S} 2-1=0 . \backslash \mathrm{S} 2 \mathrm{~A}=$ $0.750016 \backslash \mathrm{RMSD}=7.279 \mathrm{e}-09 \backslash \mathrm{RMSF}=3.241 \mathrm{e}-06 \backslash \mathrm{Dipole}=-0.1469445,-0.1666866,0$. $313772 \backslash$ Polar $=117.9609358,5.9838447,69.8165001,-1.3695049,-0.1431546,57$ $.7037004 \backslash \mathrm{PG}=\mathrm{C} 01 \quad[\mathrm{X}(\mathrm{C} 4 \mathrm{H} 6 \mathrm{~F} 3 \mathrm{~S} 2)] \backslash \backslash \mathrm{a}$

\section{$\mathrm{CH}_{3} \mathrm{~S}\left(\mathrm{CF}_{3}\right) \mathrm{C}=\mathrm{S}$}

$1 \backslash 1 \backslash G I N C-S C 13 \backslash F O p t \backslash R B 3 L Y P \backslash 6-31 G(d) \backslash C 3 H 3 F 3 S 2 \backslash D J H 501 \backslash 08-J u n-2004 \backslash 0 \backslash \backslash \#$ B3 LYP/6-31G* SCF=TIGHT OPT=TIGHT MAXDISK=65536000\\CH3SC (CF3)Se B3LYP / $6-31 \mathrm{G}(\mathrm{d})$ geom opt $\backslash \backslash 0,1 \backslash \mathrm{S}, 0.1600668171,0 .,-1.8994631176 \backslash \mathrm{C},-0.0590038526$ $, 0 .,-0.2784483338 \backslash \mathrm{C}, 1.1623100886,0.0 .6593512747 \backslash \mathrm{S},-1.5641330296,0$. 0 . $606078134 \backslash \mathrm{C},-2.786206208,0 \ldots,-0.7384060645 \backslash \mathrm{H},-3.7647348693,0 .,-0.252024$ $442 \backslash \mathrm{H},-2.6700872521,0.8913530623,-1.358775166 \backslash \mathrm{H},-2.6700872521,-0.89135$ $30623,-1.358775166 \backslash \mathrm{F}, 0.7928951805,0 ., 1.9602329358 \backslash \mathrm{F}, 1.9184062212,-1.08$ $75114514,0.4537031573 \backslash \mathrm{F}, 1.9184062212,1.0875114514,0.4537031573 \backslash \backslash$ Versio $\mathrm{n}=\mathrm{DEC}-\mathrm{AXP}-\mathrm{OSF} / 1-\mathrm{G} 03$ RevB .05 $\backslash$ State $=1-\mathrm{A}^{\prime} \backslash \mathrm{HF}=-1212.0139559 \backslash \mathrm{RMSD}=5.407 \mathrm{e}-09 \backslash$ $\mathrm{RMSF}=7.326 \mathrm{e}-06 \backslash \mathrm{Dipole}=-1.238597,0 ., 0.0073576 \backslash \mathrm{PG}=\mathrm{CS} \quad[\mathrm{SG}(\mathrm{C} 3 \mathrm{H} 1 \mathrm{~F} 1 \mathrm{~S} 2), \mathrm{X}(\mathrm{H} 2 \mathrm{~F}$ $2)] \backslash \backslash$ Q

\section{$\mathrm{CH}_{3} \mathrm{SC} \cdot\left(\mathrm{NH}_{2}\right) \mathrm{SCH}_{3}$}

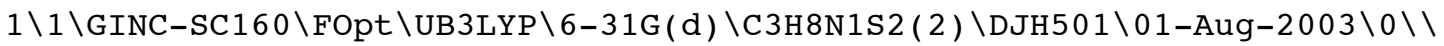
\#N UB3LYP/6-31G(D) SCF=TIGHT OPT=TIGHT MAXDISK=39321600\\CH3SC (NH2) SCH 3 a radical UB3LYP / 6-31G(d) geom opt $\backslash \backslash 0,2 \backslash N,-2.0317724087,0.023730957$ $8,0.1181493798 \backslash \mathrm{C},-0.6727873925,-0.0252932807,-0.2151600701 \backslash \mathrm{S}, 0.2106355$ $736,1.4958584317,-0.2872708422 \backslash \mathrm{C}, 0.6455286398,1.8452679886,1.478640630$ $7 \backslash \mathrm{S}, 0.1066825821,-1.5733978448,0.1803475471 \backslash \mathrm{C}, 1.2683893093,-1.73281006$ $66,-1.2260941018 \backslash \mathrm{H}, 1.8951315145,-0.8403767838,-1.2950878875 \backslash \mathrm{H}, 0.729506$ $5072,-1.8840205046,-2.1643916307 \backslash \mathrm{H}, 1.2107573531,1.0074665523,1.8937668$ $431 \backslash \mathrm{H},-0.2536127284,2.0235064462,2.0725576755 \backslash \mathrm{H}, 1.2698295127,2.7434614$ $588,1.4769111911 \backslash \mathrm{H},-2.565608069,-0.7914144382,-0.1709295489 \backslash \mathrm{H},-2.48407$ $31237,0.8960625765,-0.1352193787 \backslash \mathrm{H}, 1.8966020637,-2.6031592507,-1.01819$ $89547 \backslash \backslash$ Version=DEC-AXP-OSF $/ 1-G 03 R e v B .02 \backslash$ State $=2-A \backslash H F=-970.2140204 \backslash S 2=0$ $.754391 \backslash \mathrm{S} 2-1=0 . \backslash \mathrm{S} 2 \mathrm{~A}=0.750014 \backslash \mathrm{RMSD}=5.886 \mathrm{e}-09 \backslash \mathrm{RMSF}=6.142 \mathrm{e}-06 \backslash \mathrm{Dipole}=-0.3$ $154557,0.0328513,-0.1508421 \backslash \mathrm{PG}=\mathrm{C} 01[\mathrm{X}(\mathrm{C} 3 \mathrm{H} 8 \mathrm{~N} 1 \mathrm{~S} 2)] \backslash \backslash @$

\section{$\mathrm{CH}_{3} \mathrm{~S}\left(\mathrm{NH}_{2}\right) \mathrm{C}=\mathrm{S}$}

$1 \backslash 1 \backslash G I N C-S C 119 \backslash F r e q \backslash R B 3 L Y P \backslash 6-31 G(d) \backslash C 2 H 5 N 1 S 2 \backslash D J H 501 \backslash 16-M a y-2004 \backslash 1 \backslash \backslash \# B$ 3LYP/6-31G* SCF=TIGHT FREQ MAXDISK=52428800\\CH3SC(NH2)S B3LYP / 6-31G (d) freq $\backslash \backslash 0,1 \backslash N \backslash C, 1, B 1 \backslash S, 2, B 2,1, A 1 \backslash S, 2, B 3,1, A 2,3,180$. , 0\C, 3, B4, $2, A 3,1$, $180.0 \backslash \mathrm{H}, 5, \mathrm{~B} 5,3, \mathrm{~A} 4,2,180.0 \backslash \mathrm{H}, 5, \mathrm{~B} 6,3, \mathrm{~A} 5,6, \mathrm{D} 1,0 \backslash \mathrm{H}, 5, \mathrm{~B} 6,3, \mathrm{~A} 5,6,-\mathrm{D} 1,0 \backslash \mathrm{H}, 1$ $, \mathrm{B} 7,2, \mathrm{~A} 6,3,0 ., 0 \backslash \mathrm{H}, 1, \mathrm{~B} 8,2, \mathrm{~A} 7,9,180 ., 0 \backslash \backslash \mathrm{B} 1=1.35086232 \backslash \mathrm{B} 2=1.78936764 \backslash \mathrm{B} 3=1$ $.66174545 \backslash \mathrm{B} 4=1.81922951 \backslash \mathrm{B} 5=1.09354712 \backslash \mathrm{B} 6=1.09091636 \backslash \mathrm{B} 7=1.011685 \backslash \mathrm{B} 8=1.0$ $1047704 \backslash \mathrm{A} 1=111.06777739 \backslash \mathrm{A} 2=123.07335073 \backslash \mathrm{A} 3=102.40560811 \backslash \mathrm{A} 4=105.4566422$ $7 \backslash A 5=110.47552997 \backslash A 6=122.66517381 \backslash A 7=118.51199776 \backslash D 1=119.17507094 \backslash \backslash$ Ver sion $=D E C-A X P-O S F / 1-G 03 R e v B .05 \backslash$ State $=1-A^{\prime} \backslash H F=-930.3575603 \backslash$ RMSD $=1.856 e-0$ $9 \backslash \mathrm{RMSF}=3.231 \mathrm{e}-06 \backslash \mathrm{Dipole}=0.8655404,0,,-0.7220802 \backslash \mathrm{PG}=\mathrm{CS}[\mathrm{SG}(\mathrm{C} 2 \mathrm{H} 3 \mathrm{~N} 1 \mathrm{~S} 2), \mathrm{X}($ $\mathrm{H} 2$ ) $] \backslash \backslash \mathrm{Q}$ 


\section{$\mathrm{CH}_{3} \mathrm{SC} \cdot(\mathrm{Ph}) \mathrm{S}-\mathrm{CH}_{3}$}

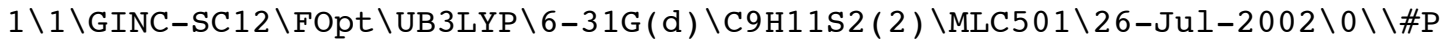
B3LYP $/ 6-31 \mathrm{G} *$ OPT $=($ MAXCYC $=100) \quad \mathrm{SCF}=(\mathrm{MAXCYC}=100) \quad$ FREQ MAXDISK $=131072000$ $\backslash \backslash \mathrm{rrad} z=\mathrm{ph} r \mathrm{r}=\mathrm{me}-\mathrm{c} 1 \mathrm{c}-6 \mathrm{duhf}-\mathrm{C} 2 \backslash \backslash 0,2 \backslash \mathrm{C}, 0,0,-0.5436243969 \backslash \mathrm{C}, 0,0 ., 0.89$ $993655 \overline{5} 8 \backslash \mathrm{C}, \overline{1} .2045767983,-0.1407684102,1.6447473811 \backslash \mathrm{C},-1.2045767983,0.1$ $407684102,1.6447473811 \backslash \mathrm{C}, 1.1980023223,-0.1372926446,3.0327870849 \backslash \mathrm{C},-1$. $1980023223,0.1372926446,3.0327870849 \backslash \mathrm{H}, 2.1407212827,-0.2659311883,1.11$ $24152303 \backslash \mathrm{H},-2.1407212827,0.2659311883,1.1124152303 \backslash \mathrm{H}, 2.1367874102,-0.2$ $51386417,3.5689285696 \backslash \mathrm{H},-2.1367874102,0.251386417,3.5689285696 \backslash \mathrm{C}, 0 ., 0$. $, 3.7425798751 \backslash \mathrm{H}, 0 ., 0 ., 4.828948414 \backslash \mathrm{S},-1.5041998417,-0.2727499725,-1.435$ $6947229 \backslash \mathrm{C},-1.5483731257,1.1133863252,-2.639460946 \backslash \mathrm{H},-2.4327150559,0.94$ $51928411,-3.2601525901 \backslash \mathrm{H},-1.6417073267,2.0701943282,-2.120752452 \backslash \mathrm{H},-0$. $656494302,1.1210366726,-3.2689146201 \backslash \mathrm{S}, 1.5041998417,0.2727499725,-1.43$ $56947229 \backslash \mathrm{C}, 1.5483731257,-1.1133863252,-2.639460946 \backslash \mathrm{H}, 2.4327150559,-0.9$ $451928411,-3.2601525901 \backslash \mathrm{H}, 1.6417073267,-2.0701943282,-2.120752452 \backslash \mathrm{H}, 0$. $656494302,-1.1210366726,-3.2689146201 \backslash \backslash$ Version=DEC-AXP-OSF / 1-G98RevA. 1 $1.3 \backslash$ State $=2-B \backslash H F=-1145.9239982 \backslash \mathrm{S} 2=0.772251 \backslash \mathrm{S} 2-1=0 . \backslash \mathrm{S} 2 \mathrm{~A}=0.750367 \backslash \mathrm{RMSD}=6$ $.432 \mathrm{e}-09 \backslash \mathrm{RMSF}=1.039 \mathrm{e}-05 \backslash \mathrm{Dipole}=0 ., 0 .,-0.434059 \backslash \mathrm{PG}=\mathrm{C} 02 \quad[\mathrm{C} 2(\mathrm{C} 1 \mathrm{C} 1 \mathrm{C} 1 \mathrm{H} 1), \mathrm{X}($ $\mathrm{C} 6 \mathrm{H} 10 \mathrm{~S} 2) \mathrm{J} \backslash \backslash @$

\section{$\mathrm{S}=\mathrm{C}(\mathrm{Ph}) \mathrm{SCH}_{3}$}

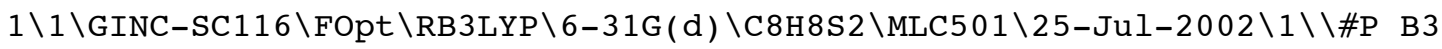
LYP $/ 6-31 \mathrm{G} *$ OPT $=(\mathrm{Z}-\mathrm{MATRIX}, \mathrm{MAXCYC}=100) \quad$ FREQ MAXDISK $=131072000 \backslash \backslash \mathrm{RAFT}$ agen t $S=C(P h)-S-C H 3 \quad(Z=p h, R=m e) \backslash \backslash 0,1 \backslash C \backslash S, 1, B 1 \backslash S, 1, B 2,2, A 1 \backslash C, 3, B 3,1, A 2,2, D 1$ $, 0 \backslash \mathrm{H}, 4, \mathrm{~B} 4,3, \mathrm{~A} 3,1, \mathrm{D} 2,0 \backslash \mathrm{H}, 4, \mathrm{~B} 5,3, \mathrm{~A} 4,5, \mathrm{D} 3,0 \backslash \mathrm{H}, 4, \mathrm{~B} 6,3, \mathrm{~A} 5,5, \mathrm{D} 4,0 \backslash \mathrm{C}, 1, \mathrm{~B} 7,2, \mathrm{~A}$ $6,3, \mathrm{D} 5,0 \backslash \mathrm{C}, 8, \mathrm{~B} 8,1, \mathrm{~A} 7,2, \mathrm{D} 6,0 \backslash \mathrm{C}, 9, \mathrm{~B} 9,8, \mathrm{~A} 8,1, \mathrm{D} 7,0 \backslash \mathrm{C}, 10, \mathrm{~B} 10,9, \mathrm{~A} 9,8, \mathrm{D} 8,0 \backslash \mathrm{C}$, $11, \mathrm{~B} 11,10, \mathrm{~A} 10,9, \mathrm{D} 9,0 \backslash \mathrm{C}, 12, \mathrm{~B} 12,11, \mathrm{~A} 11,10, \mathrm{D} 10,0 \backslash \mathrm{H}, 13, \mathrm{~B} 13,12, \mathrm{~A} 12,11, \mathrm{D} 11,0$ $\backslash \mathrm{H}, 12, \mathrm{~B} 14,11, \mathrm{~A} 13,13, \mathrm{D} 12,0 \backslash \mathrm{H}, 11, \mathrm{~B} 15,10, \mathrm{~A} 14,12, \mathrm{D} 13,0 \backslash \mathrm{H}, 10, \mathrm{~B} 16,9, \mathrm{~A} 15,11$, D $14,0 \backslash \mathrm{H}, 9, \mathrm{~B} 17,8, \mathrm{~A} 16,10, \mathrm{D} 15,0 \backslash \backslash \mathrm{B} 1=1.65390665 \backslash \mathrm{B} 2=1.76783874 \backslash \mathrm{B} 3=1.81735533$ $\backslash \mathrm{B} 4=1.09340644 \backslash \mathrm{B} 5=1.09203382 \backslash \mathrm{B} 6=1.09167768 \backslash \mathrm{B} 7=1.48638437 \backslash \mathrm{B} 8=1.40791986$ $\backslash \mathrm{B} 9=1.3934782 \backslash \mathrm{B} 10=1.3954032 \backslash \mathrm{B} 11=1.39756856 \backslash \mathrm{B} 12=1.39093249 \backslash \mathrm{B} 13=1.084558$ $18 \backslash \mathrm{B} 14=1.08646979 \backslash \mathrm{B} 15=1.08663117 \backslash \mathrm{B} 16=1.08643383 \backslash \mathrm{B} 17=1.08519217 \backslash \mathrm{A} 1=123$. $8831348 \backslash \mathrm{A} 2=103.44785578 \backslash \mathrm{A} 3=105.74311227 \backslash \mathrm{A} 4=110.56367906 \backslash \mathrm{A} 5=110.5050450$ $1 \backslash \mathrm{A} 6=123.43517028 \backslash \mathrm{A} 7=121.7784707 \backslash \mathrm{A} 8=120.59384832 \backslash \mathrm{A} 9=120.24074703 \backslash \mathrm{A} 10=1$ $19.68786961 \backslash \mathrm{A} 11=120.27839448 \backslash \mathrm{A} 12=120.55619114 \backslash \mathrm{A} 13=120.1204252 \backslash \mathrm{A} 14=120$. $14506072 \backslash A 15=119.5622211 \backslash A 16=119.91357919 \backslash D 1=5.07451867 \backslash D 2=178.7658797$ $5 \backslash D 3=-119.43094734 \backslash D 4=119.41965567 \backslash D 5=181.27901545 \backslash D 6=211.48944504 \backslash D 7=$ $179.74862327 \backslash \mathrm{D} 8=0.5087189 \backslash \mathrm{D} 9=-0.47334322 \backslash \mathrm{D} 10=-0.1123593 \backslash \mathrm{D} 11=180.118421$ $67 \backslash D 12=179.95923577 \backslash D 13=180.24754199 \backslash D 14=180.47358662 \backslash D 15=182.12059766$ \\Version=DEC-AXP-OSF $/ 1-G 98 R e v A .11 .3 \backslash H F=-1106.0452545 \backslash R M S D=3.885 e-09 \backslash R$ $\mathrm{MSF}=1.915 \mathrm{e}-05 \backslash \mathrm{Dipole}=0.0516049,-0.0290079,-0.8060901 \backslash \mathrm{PG}=\mathrm{C} 01 \quad[\mathrm{X}(\mathrm{C} 8 \mathrm{H} 8 \mathrm{~S} 2)$ ]$\backslash \backslash @$

\section{$\mathrm{CH}_{3} \mathrm{SC} \cdot\left(\mathrm{CH}_{2} \mathrm{Ph}\right) \mathrm{S}-\mathrm{CH}_{3}$}

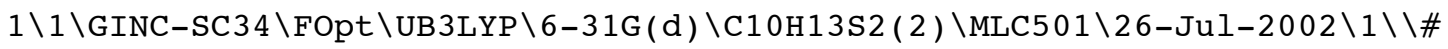
P UB3LYP/6-31G(D) FOPT=(Z-MATRIX, MAXCYC=100) FREQ MAXDISK $=131072000 \backslash \backslash \mathrm{R}$

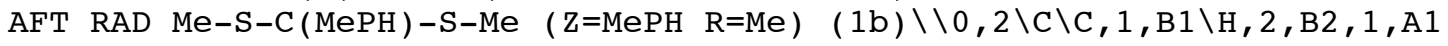
$\backslash \mathrm{C}, 2, \mathrm{~B} 3,1, \mathrm{~A} 2,3, \mathrm{D} 1,0 \backslash \mathrm{C}, 4, \mathrm{~B} 4,2, \mathrm{~A} 3,1, \mathrm{D} 2,0 \backslash \mathrm{C}, 5, \mathrm{~B} 5,4, \mathrm{~A} 4,2, \mathrm{D} 3,0 \backslash \mathrm{C}, 6, \mathrm{~B} 6,5, \mathrm{~A} 5$, $4, \mathrm{D} 4,0 \backslash \mathrm{C}, 7, \mathrm{~B} 7,6, \mathrm{~A} 6,5, \mathrm{D} 5,0 \backslash \mathrm{C}, 8, \mathrm{~B} 8,7, \mathrm{~A} 7,6, \mathrm{D} 6,0 \backslash \mathrm{H}, 9, \mathrm{~B} 9,8, \mathrm{~A} 8,7, \mathrm{D} 7,0 \backslash \mathrm{H}, 8, \mathrm{~B} 1$ $0,7, \mathrm{~A} 9,9, \mathrm{D} 8,0 \backslash \mathrm{H}, 7, \mathrm{~B} 11,6, \mathrm{~A} 10,8, \mathrm{D} 9, \mathrm{O} \backslash \mathrm{H}, 6, \mathrm{~B} 12,5, \mathrm{~A} 11,7, \mathrm{D} 10,0 \backslash \mathrm{H}, 5, \mathrm{~B} 13,4, \mathrm{~A} 12$

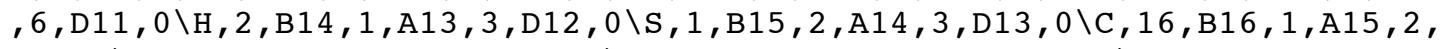
$\mathrm{D} 14,0 \backslash \mathrm{H}, 17, \mathrm{~B} 17,16, \mathrm{~A} 16,1, \mathrm{D} 15,0 \backslash \mathrm{H}, 17, \mathrm{~B} 18,16, \mathrm{~A} 17,18, \mathrm{D} 16,0 \backslash \mathrm{H}, 17, \mathrm{~B} 19,16, \mathrm{~A} 18$

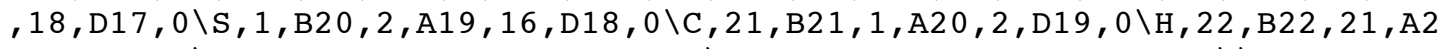
$1,1, \mathrm{D} 20,0 \backslash \mathrm{H}, 22, \mathrm{~B} 23,21, \mathrm{~A} 22,23, \mathrm{D} 21,0 \backslash \mathrm{H}, 22, \mathrm{~B} 24,21, \mathrm{~A} 23,23, \mathrm{D} 22,0 \backslash \backslash \mathrm{B} 1=1.5157$ $8873 \backslash \mathrm{B} 2=1.09765845 \backslash \mathrm{B} 3=1.52389414 \backslash \mathrm{B} 4=1.40058996 \backslash \mathrm{B} 5=1.39681842 \backslash \mathrm{B} 6=1.3955$ $8668 \backslash B 7=1.39697813 \backslash B 8=1.39470243 \backslash B 9=1.08777803 \backslash B 10=1.08705661 \backslash B 11=1.08$ $678408 \backslash \mathrm{B} 12=1.08707699 \backslash \mathrm{B} 13=1.08729883 \backslash \mathrm{B} 14=1.09778413 \backslash \mathrm{B} 15=1.75737506 \backslash \mathrm{B} 16$ 
$=1.8511538 \backslash \mathrm{B} 17=1.09395743 \backslash \mathrm{B} 18=1.09286269 \backslash \mathrm{B} 19=1.09134704 \backslash \mathrm{B} 20=1.75969536$ $\backslash \mathrm{B} 21=1.82647684 \backslash \mathrm{B} 22=1.09192111 \backslash \mathrm{B} 23=1.09332514 \backslash \mathrm{B} 24=1.09120267 \backslash \mathrm{A} 1=108.25$ $525698 \backslash \mathrm{A} 2=113.94938997 \backslash \mathrm{A} 3=120.79198662 \backslash \mathrm{A} 4=120.78731 \backslash \mathrm{A} 5=120.13467307 \backslash \mathrm{A} 6$ $=119.55192775 \backslash A 7=120.16376976 \backslash A 8=119.72340424 \backslash A 9=120.07880369 \backslash A 10=120$. $24221717 \backslash A 11=119.74087848 \backslash A 12=119.32184346 \backslash A 13=109.03557488 \backslash A 14=120.36$ $439381 \backslash A 15=104.08221211 \backslash A 16=106.22727959 \backslash A 17=110.79627524 \backslash A 18=110.4665$ $5003 \backslash A 19=115.46042477 \backslash A 20=104.60546169 \backslash A 21=110.57101967 \backslash A 22=105.689005$ $73 \backslash \mathrm{A} 23=111.64284706 \backslash \mathrm{D} 1=-121.45670912 \backslash \mathrm{D} 2=-261.77203284 \backslash \mathrm{D} 3=181.36035309 \backslash$ $\mathrm{D} 4=-0.2080065 \backslash \mathrm{D} 5=-0.01087986 \backslash \mathrm{D} 6=0.19371478 \backslash \mathrm{D} 7=-180.12483316 \backslash \mathrm{D} 8=180.168$ $86927 \backslash D 9=180.28334206 \backslash D 10=-179.64263638 \backslash D 11=179.92707274 \backslash D 12=115.75269$ $857 \backslash D 13=49.66353815 \backslash D 14=96.41159877 \backslash D 15=175.79130312 \backslash D 16=-118.67140069$ $\backslash \mathrm{D} 17=119.10764327 \backslash \mathrm{D} 18=170.17863131 \backslash \mathrm{D} 19=166.2284763 \backslash \mathrm{D} 20=-57.73757008 \backslash \mathrm{D} 2$ $1=-118.66229821 \backslash \mathrm{D} 22=122.32870879 \backslash \backslash$ Version=DEC-AXP-OSF $/ 1-G 98 \mathrm{RevA} .11 .3 \backslash \mathrm{H}$ $\mathrm{F}=-1185.2251153 \backslash \mathrm{S} 2=0.755322 \backslash \mathrm{S} 2-1=0 . \backslash \mathrm{S} 2 \mathrm{~A}=0.750022 \backslash \mathrm{RMSD}=2.912 \mathrm{e}-09 \backslash \mathrm{RMSF}=4$ $.358 e-05 \backslash \mathrm{Dipole}=-0.3028549,-0.2300317,-0.3558071 \backslash \mathrm{PG}=\mathrm{C} 01[\mathrm{X}(\mathrm{C} 10 \mathrm{H} 13 \mathrm{~S} 2)] \backslash$ 10

\section{$\mathrm{S}=\mathrm{C}\left(\mathrm{CH}_{2} \mathrm{Ph}\right) \mathrm{SCH}_{3}$}

$1 \backslash 1 \backslash G I N C-S C 116 \backslash F O p t \backslash R B 3 L Y P \backslash 6-31 G(d) \backslash C 9 H 10 S 2 \backslash M L C 501 \backslash 25-J u l-2002 \backslash 1 \backslash \backslash \# P$ B 3LYP/6-31G* OPT $=(Z-M A T R I X, M A X C Y C=100)$ FREQ MAXDISK=131072000\\RAFT Age nt $\mathrm{S}=\mathrm{C}(\mathrm{MePH})-\mathrm{SMe}(\mathrm{Z}=\mathrm{MePh}, \mathrm{R}=\mathrm{Me}) \mathrm{C} 1 \backslash \backslash 0,1 \backslash \mathrm{C} \backslash \mathrm{S}, 1, \mathrm{~B} 1 \backslash \mathrm{C}, 1, \mathrm{~B} 2,2, \mathrm{~A} 1 \backslash \mathrm{H}, 3, \mathrm{~B} 3,1$, $\mathrm{A} 2,2, \mathrm{D} 1,0 \backslash \mathrm{H}, 3, \mathrm{~B} 4,1, \mathrm{~A} 3,4, \mathrm{D} 2,0 \backslash \mathrm{C}, 3, \mathrm{~B} 5,1, \mathrm{~A} 4,4, \mathrm{D} 3,0 \backslash \mathrm{C}, 6, \mathrm{~B} 6,3, \mathrm{~A} 5,1, \mathrm{D} 4,0 \backslash \mathrm{C}, 7$ , $\mathrm{B} 7,6, \mathrm{~A} 6,3, \mathrm{D} 5,0 \backslash \mathrm{C}, 8, \mathrm{~B} 8,7, \mathrm{~A} 7,6, \mathrm{D} 6,0 \backslash \mathrm{C}, 9, \mathrm{~B} 9,8, \mathrm{~A} 8,7, \mathrm{D} 7,0 \backslash \mathrm{C}, 10, \mathrm{~B} 10,9, \mathrm{~A} 9,8$, $\mathrm{D} 8,0 \backslash \mathrm{H}, 11, \mathrm{~B} 11,10, \mathrm{~A} 10,9, \mathrm{D} 9,0 \backslash \mathrm{H}, 10, \mathrm{~B} 12,9, \mathrm{~A} 11,11, \mathrm{D} 10,0 \backslash \mathrm{H}, 9, \mathrm{~B} 13,8, \mathrm{~A} 12,10, \mathrm{D}$ $11,0 \backslash \mathrm{H}, 8, \mathrm{~B} 14,7, \mathrm{~A} 13,9, \mathrm{D} 12,0 \backslash \mathrm{H}, 7, \mathrm{~B} 15,6, \mathrm{~A} 14,8, \mathrm{D} 13,0 \backslash \mathrm{S}, 1, \mathrm{~B} 16,2, \mathrm{~A} 15,3, \mathrm{D} 14,0$ $\backslash \mathrm{C}, 17, \mathrm{~B} 17,1, \mathrm{~A} 16,2, \mathrm{D} 15,0 \backslash \mathrm{H}, 18, \mathrm{~B} 18,16, \mathrm{~A} 17,1, \mathrm{D} 16,0 \backslash \mathrm{H}, 18, \mathrm{~B} 19,16, \mathrm{~A} 18,1, \mathrm{D} 17$, $0 \backslash \mathrm{H}, 18, \mathrm{~B} 20,16, \mathrm{~A} 19,1, \mathrm{D} 18,0 \backslash \backslash \mathrm{B} 1=1.64564466 \backslash \mathrm{B} 2=1.52863415 \backslash \mathrm{B} 3=1.09674628 \backslash \mathrm{B}$ $4=1$. $09353236 \backslash B 5=1.52343747 \backslash B 6=1.39968158 \backslash B 7=1.39594042 \backslash B 8=1.39529816 \backslash B$ $9=1.39667801 \backslash \mathrm{B} 10=1.3946779 \backslash \mathrm{B} 11=1.08659727 \backslash \mathrm{B} 12=1.08682646 \backslash \mathrm{B} 13=1.0867289$ $4 \backslash \mathrm{B} 14=1.08683319 \backslash \mathrm{B} 15=1.08756909 \backslash \mathrm{B} 16=1.7547672 \backslash \mathrm{B} 17=1.81824902 \backslash \mathrm{B} 18=1.093$ $09254 \backslash B 19=1.09194447 \backslash B 20=1.0919036 \backslash A 1=123.05916516 \backslash A 2=109.80486391 \backslash A 3=$ $107.35736989 \backslash A 4=112.35357201 \backslash A 5=120.55086294 \backslash A 6=120.654591 \backslash A 7=120.0667$ $1159 \backslash A 8=119.64159031 \backslash A 9=120.22881755 \backslash A 10=120.05599167 \backslash A 11=120.09413459$ $\backslash \mathrm{A} 12=120.18319366 \backslash \mathrm{A} 13=119.73589867 \backslash \mathrm{A} 14=119.61324586 \backslash \mathrm{A} 15=125.66992548 \backslash \mathrm{A}$ $16=103.79835693 \backslash A 17=105.33482935 \backslash A 18=93.82431614 \backslash A 19=126.14621 \backslash D 1=150$. $7138342 \backslash D 2=-116.89084223 \backslash D 3=121.89888903 \backslash D 4=-100.14574145 \backslash D 5=179.75805$ $35 \backslash D 6=-0.07990219 \backslash D 7=0.06511684 \backslash D 8=-0.01890592 \backslash D 9=180.33270637 \backslash D 10=179$ $.82110496 \backslash D 11=-180.19616308 \backslash D 12=179.8418732 \backslash D 13=179.81914239 \backslash D 14=-178$. $68759297 \backslash D 15=2.34439814 \backslash D 16=197.73877987 \backslash D 17=85.44790483 \backslash D 18=-31.96744$ 553 \Version=DEC-AXP-OSF / 1-G98RevA. 11.3\HF=-1145.3555037 \RMSD=4 . $812 \mathrm{e}-0$ $9 \backslash \mathrm{RMSF}=1.589 \mathrm{e}-05 \backslash \mathrm{Dipole}=-0.341854,0.1539887,-0.6355984 \backslash \mathrm{PG}=\mathrm{C} 01] \mathrm{X}(\mathrm{C} 9 \mathrm{H} 10$ S2) $] \backslash \backslash @$

\section{$\mathrm{CH}_{3} \mathrm{SC} \cdot\left(\mathrm{OCH}_{3}\right) \mathrm{SCH}_{3}$}

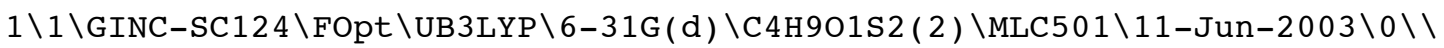
\# B3LYP/6-31G* OPT $=($ MAXCYC $=100)$ FREQ MAXDISK $=13107200$ GEOM=CHECK GUESS $=\mathrm{READ} \backslash \backslash \mathrm{rrad} z=0 \mathrm{me} r=\mathrm{me}-\mathrm{a} 1 \mathrm{~b} 1 \mathrm{c} 1 \mathrm{~d} 3 \mathrm{e} 2 \mathrm{f} 1-6 \mathrm{dub} 3 \backslash \backslash 0,2 \backslash \mathrm{S},-1.3410354108,-1.1021$ $645587,0.00 \overline{8} 37807 \overline{2} 2 \backslash \mathrm{C},-0.0668314555,0.0846927765,-0.2625382649 \backslash \mathrm{S}, 1.525$ $0665723,-0.5327357432,-0.7113926887 \backslash \mathrm{C}, 2.3426793192,-0.7855867589,0.929$ $7015005 \backslash \mathrm{C},-2.8220656021,-0.0301869566,0.1187230656 \backslash 0,-0.1829188202,1.2$ $334385003,0.4787862469 \backslash \mathrm{C}, 0.3588901181,2.4188570239,-0.1145681207 \backslash \mathrm{H},-2$. $9779210372,0.5082852285,-0.8196451416 \backslash \mathrm{H},-3.670068575,-0.6955390127,0.2$ $998754487 \backslash \mathrm{H},-2.72432788,0.675718492,0.9443720255 \backslash \mathrm{H}, 2.2450048802,0.1147$ $964264,1.5398219577 \backslash \mathrm{H}, 3.400214885,-0.9780596806,0.7296543233 \backslash \mathrm{H}, 1.90496$ $92578,-1.6399132978,1.4510056405 \backslash \mathrm{H}, 0.1716693923,3.2223284691,0.6006696$ $643 \backslash \mathrm{H}, 1.4348670988,2.3181216146,-0.2907133132 \backslash \mathrm{H},-0.1415903241,2.638502$ $0801,-1.0650057993 \backslash \backslash$ Version=DEC $-\mathrm{AXP}-\mathrm{OSF} / 1-\mathrm{G} 03 \mathrm{RevB} .03 \backslash$ State $=2-\mathrm{A} \backslash \mathrm{HF}=-102$ 
$9.3786222 \backslash \mathrm{S} 2=0.754676 \backslash \mathrm{S} 2-1=0 . \backslash \mathrm{S} 2 \mathrm{~A}=0.750016 \backslash \mathrm{RMSD}=3.068 \mathrm{e}-09 \backslash \mathrm{RMSF}=1.311 \mathrm{e}-$ $06 \backslash \mathrm{Dipole}=-0.1181936,0.8010544,0.3889717 \backslash \mathrm{PG}=\mathrm{C} 01 \quad[\mathrm{X}(\mathrm{C} 4 \mathrm{H} 901 \mathrm{~S} 2)] \backslash \backslash @$

\section{$\mathrm{S}=\mathrm{C}\left(\mathrm{OCH}_{3}\right) \mathrm{SCH}_{3}$}

$1 \backslash 1 \backslash G I N C-S C 160 \backslash F O p t \backslash R B 3 L Y P \backslash 6-31 G(d) \backslash C 3 H 601 S 2 \backslash M L C 501 \backslash 28-A p r-2003 \backslash 0 \backslash \backslash \# N$ B3LYP/6-31G(D) OPT=(TIGHT, MAXCYC=100) FREQ MAXDISK=131072000 GEOM=CHEC $\mathrm{K}$ GUESS $=\mathrm{READ} \backslash \backslash \mathrm{RAFT}(\mathrm{Z}=\mathrm{OMe} \mathrm{R}=\mathrm{CH} 3)$ lowest $\mathrm{a} 1 \mathrm{~b} 1$ in $\mathrm{Cs}$ symm $\backslash \backslash 0,1 \backslash \mathrm{C}, 0.374209$ $3441,-2.4921458868,0 . \backslash S, 1.301426637,-0.9260065359,0$. \C, $-0.0000019362,0$ $.2785978098,0 . \backslash \mathrm{S},-1.6256039629,-0.0059529119,0 . \backslash 0,0.5982011851,1.47426$ $53379,0 . \backslash \mathrm{C},-0.2142814517,2.6603482395,0 . \backslash \mathrm{H}, 1.1323627109,-3.280322659,0$ $. \backslash \mathrm{H},-0.2490760809,-2.5725262915,0.8922739391 \backslash \mathrm{H},-0.2490760809,-2.572526$ $2915,-0.8922739391 \backslash \mathrm{H}, 0.4987378961,3.485149722,0 . \backslash \mathrm{H},-0.8456382241,2.688$ $326503,-0.8916364756 \backslash \mathrm{H},-0.8456382241,2.688326503,0.8916364756 \backslash \backslash$ Version =DEC-AXP-OSF / 1-G98RevA. 11.3 \State=1-A ' $\backslash \mathrm{HF}=-989.5242355 \backslash \mathrm{RMSD}=5.328 \mathrm{e}-09 \backslash$ $\mathrm{RMSF}=3.625 \mathrm{e}-06 \backslash \mathrm{Dipole}=0.2790653,0.1622872,0 . \backslash \mathrm{PG}=\mathrm{CS}[\mathrm{SG}(\mathrm{C} 3 \mathrm{H} 2 \mathrm{O} 1 \mathrm{~S} 2), \mathrm{X}(\mathrm{H} 4)$ ]$\backslash \backslash Q$

\section{$\mathrm{CH}_{3} \mathrm{SC} \cdot\left(\mathrm{OCH}_{2} \mathrm{CH}_{3}\right) \mathrm{SCH}_{3}$}

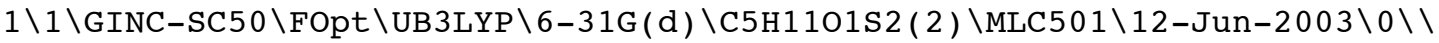
\# B3LYP/6-31G* OPT= $($ MAXCYC $=100)$ FREQ MAXDISK $=134217728$ GEOM=CHECK GUES $\mathrm{S}=\mathrm{READ} \backslash \backslash \mathrm{rrad} z=$ oet_r=me-a1b1c1d3e2f $1-6 \mathrm{dub} 3 \backslash \backslash 0,2 \backslash \mathrm{S}, 1.7423907621,-0.9654$ $242546,0.128 \overline{0} 80256 \overline{4} \backslash \mathrm{C}, 0.3393190694,0.0076784401,-0.3116956685 \backslash \mathrm{S}, 0.6303$ $178003,1.7122689272,-0.6718428413 \backslash \mathrm{C}, 0.4396160625,2.5167306849,0.983741$ $8624 \backslash \mathrm{C}, 1.0419958484,-2.6568847766,0.081707205 \backslash 0,-0.8417886969,-0.39199$ $54224,0.2566619351 \backslash \mathrm{C},-2.0409053693,-0.1258027406,-0.4989653844 \backslash \mathrm{H}, 0.698$ $0673109,-2.9039347333,-0.9259399649 \backslash \mathrm{H}, 1.8526385793,-3.3333648574,0.363$ $9360802 \backslash \mathrm{H}, 0.2195155158,-2.7506488733,0.7920901202 \backslash \mathrm{H},-0.4988810008,2.20$ $4729172,1.4468746738 \backslash \mathrm{H}, 0.4197718259,3.5961627799,0.8106289169 \backslash \mathrm{H}, 1.2795$ $067643,2.2635964141,1.6346466759 \backslash \mathrm{C},-3.2099223896,-0.6971972568,0.28261$ $14787 \backslash \mathrm{H},-1.9487825653,-0.5924504824,-1.4882299133 \backslash \mathrm{H},-2.1466192075,0.95$ $52698805,-0.646297879 \backslash \mathrm{H},-4.145906282,-0.5121722605,-0.255684689 \backslash \mathrm{H},-3.0$ $976073558,-1.7774777593,0.420113456 \backslash \mathrm{H},-3.2813503359,-0.2304067694,1.27$ $03714428 \backslash \backslash$ Version=DEC-AXP-OSF /1-G03RevB.03\State $=2-A \backslash H F=-1068.6978609 \backslash$ $\mathrm{S} 2=0.754682 \backslash \mathrm{S} 2-1=0 . \backslash \mathrm{S} 2 \mathrm{~A}=0.750016 \backslash \mathrm{RMSD}=4.362 \mathrm{e}-09 \backslash \mathrm{RMSF}=4.589 \mathrm{e}-05 \backslash \mathrm{Dipole}=$ $-0.8687421,-0.3333734,0.2996148 \backslash \mathrm{PG}=\mathrm{C} 01[\mathrm{X}(\mathrm{C} 5 \mathrm{H} 1101 \mathrm{~S} 2)] \backslash \backslash \mathrm{a}$

\section{$\mathrm{S}=\mathrm{C}\left(\mathrm{OCH}_{2} \mathrm{CH}_{3}\right) \mathrm{SCH}_{3}$}

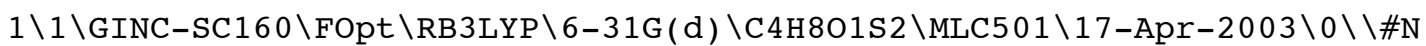

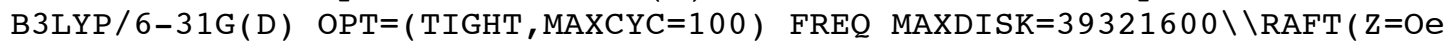
$t \mathrm{R}=\mathrm{Me}) \mathrm{a}$ b1 $\mathrm{Cs} \backslash \backslash 0,1 \backslash 0,-1.1437055573,-0.0071493756,0 . \backslash \mathrm{C}, 0.1908056904,0$ $.0159885534,0 . \backslash \mathrm{S}, 1.1547744866,1.3566881401,0 . \backslash \mathrm{S}, 0.7173978682,-1.679486$ $5418,0 . \backslash \mathrm{C},-1.881632827,1.2406966192,0 . \backslash \mathrm{C}, 2.530332313,-1.5196200975,0 . \backslash$ $\mathrm{H}, 2.9139622205,-2.5438259958,0 . \backslash \mathrm{H}, 2.8722378969,-0.9919429029,-0.892046$ $9855 \backslash \mathrm{H}, 2.8722378969,-0.9919429029,0.8920469855 \backslash \mathrm{C},-3.3553265843,0.88286$ $80162,0 . \backslash \mathrm{H},-1.5898648833,1.8145031619,-0.8849261225 \backslash \mathrm{H},-1.5898648833,1$. $8145031619,0.8849261225 \backslash \mathrm{H},-3.9527537929,1.8009113963,0 . \backslash \mathrm{H},-3.618069613$ $, 0.3000824834,-0.8882588427 \backslash \mathrm{H},-3.618069613,0.3000824834,0.8882588427 \backslash \backslash$ Version=DEC-AXP-OSF / 1-G98RevA.11.3 State $=1-A^{\prime} \backslash \mathrm{HF}=-1028.8435755 \backslash \mathrm{RMSD}=6$. $745 \mathrm{e}-09 \backslash \mathrm{RMSF}=2.655 \mathrm{e}-06 \backslash \mathrm{Dipole}=-0.403286,-0.187352,0 . \backslash \mathrm{PG}=\mathrm{CS} \quad[\mathrm{SG}(\mathrm{C} 4 \mathrm{H} 2 \mathrm{O} 1 \mathrm{~S}$ $2), \mathrm{X}(\mathrm{H} 6) \mathrm{l} \backslash \backslash \mathrm{Q}$

\section{$\mathrm{CH}_{3} \mathrm{SC} \cdot\left(\mathrm{OCH}\left(\mathrm{CH}_{3}\right)_{2}\right) \mathrm{SCH}_{3}$}

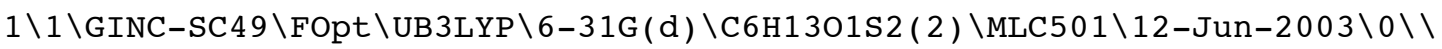
\# B3LYP/6-31G* OPT= $(\mathrm{MAXCYC}=100)$ FREQ MAXDISK=134217728 GEOM=CHECK GUES $\mathrm{S}=\mathrm{READ} \backslash \backslash \mathrm{rrad} z=\mathrm{zpr} r=\mathrm{me}-\mathrm{a} 1 \mathrm{~b} 1 \mathrm{c} 1 \mathrm{~d} 3 \mathrm{e} 1 \mathrm{f} 1-6 \mathrm{dub} 3 \backslash \backslash 0,2 \backslash \mathrm{S}, 1.6328019823,1.38840$ $1723,-0.104989024 \backslash \bar{C}, 0.4962064126,0.0853734886,0.2517209429 \backslash \mathrm{S}, 1.1988888$ $212,-1.4468685145,0.7847054864 \backslash \mathrm{C}, 1.5098017786,-2.3335346769,-0.8095250$ 
$991 \backslash \mathrm{C}, 0.5054636028,2.8082485426,-0.3591364913 \backslash 0,-0.6550688454,0.101328$ $8175,-0.490018896 \backslash \mathrm{C},-1.8541633765,-0.4485441202,0.1239514117 \backslash \mathrm{H},-0.0546$ $290771,3.0257076897,0.5539093457 \backslash \mathrm{H}, 1.1402618391,3.662826288,-0.6069598$ $626 \backslash \mathrm{H},-0.1826352,2.6091653962,-1.1816575362 \backslash \mathrm{H}, 0.6082856175,-2.32249988$ $96,-1.4256007749 \backslash \mathrm{H}, 1.7644760353,-3.3656333436,-0.5536231302 \backslash \mathrm{H}, 2.338988$ $0911,-1.8731468445,-1.3515149837 \backslash \mathrm{C},-2.8466427355,-0.6420464264,-1.0142$ $666934 \backslash \mathrm{C},-2.3630065028,0.4846642067,1.2182888647 \backslash \mathrm{H},-1.5907540546,-1.41$ $91770644,0.5610261847 \backslash \mathrm{H},-3.7739747164,-1.0858305628,-0.6364213457 \backslash \mathrm{H},-3$ $.0893532393,0.3187454166,-1.4818233384 \backslash \mathrm{H},-2.4333910582,-1.3033608673,-$ $1.7822933569 \backslash \mathrm{H},-3.2544104671,0.0613944008,1.6949712795 \backslash \mathrm{H},-1.5986473683$ $, 0.6288005873,1.9885485942 \backslash \mathrm{H},-2.6266735704,1.4628808307,0.7999290815 \backslash \backslash$ Version=DEC-AXP-OSF $/ 1-G 03 R e v B .03 \backslash$ State $=2-A \backslash H F=-1108.0164465 \backslash \mathrm{S} 2=0.75462$ $8 \backslash \mathrm{S} 2-1=0 . \backslash \mathrm{S} 2 \mathrm{~A}=0.750016 \backslash \mathrm{RMSD}=4.287 \mathrm{e}-09 \backslash \mathrm{RMSF}=3.572 \mathrm{e}-06 \backslash \mathrm{Dipole}=-0.8829033$ $, 0.0665324,-0.4079764 \backslash \mathrm{PG}=\mathrm{C} 01[\mathrm{X}(\mathrm{C} 6 \mathrm{H} 1301 \mathrm{~S} 2)] \backslash \backslash @$

\section{$\mathrm{S}=\mathrm{C}\left(\mathrm{OCH}\left(\mathrm{CH}_{3}\right)_{2}\right) \mathrm{SCH}_{3}$}

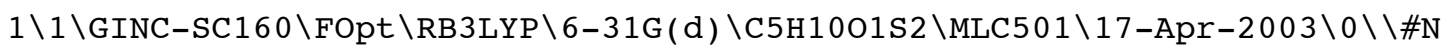
$\mathrm{B} 3 \mathrm{LYP} / 6-31 \mathrm{G}(\mathrm{D}) \mathrm{OPT}=(\mathrm{TIGHT}, \mathrm{MAXCYC}=100)$ FREQ MAXDISK=39321600 $\backslash \mathrm{RAFT}(\mathrm{Z}=0$ pr $\mathrm{R}=\mathrm{Me}) \mathrm{a} 1 \mathrm{~b} 1 \backslash \backslash 0,1 \backslash 0,-0.1880409305,-0.1960063398,-0.9146059654 \backslash \mathrm{C},-0.16$ $34698107,0.1208318952,0.3796378951 \backslash \mathrm{S}, 1.0989419575,0.7518398963,1.24169$ $43287 \backslash \mathrm{S},-1.7804771858,-0.2656031225,1.0071850584 \backslash \mathrm{C}, 0.9905955826,-0.022$ $3839841,-1.7694161684 \backslash \mathrm{C},-1.6426563686,0.2008613435,2.760975016 \backslash \mathrm{H},-0.86$ $51536385,-0.3828064605,3.256969389 \backslash \mathrm{H},-1.4238995035,1.2651146149,2.8631$ $670358 \backslash \mathrm{H},-2.6185788355,-0.0249825208,3.2001517599 \backslash \mathrm{C}, 0.4424182525,0.298$ $0059902,-3.1523519357 \backslash \mathrm{C}, 1.8249584935,-1.2958520616,-1.716083723 \backslash \mathrm{H}, 1.55$ $88488582,0.8208918648,-1.3688382623 \backslash \mathrm{H}, 1.2719818992,0.456344208,-3.8498$ $534733 \backslash \mathrm{H},-0.173003433,-0.5258789427,-3.5292417693 \backslash \mathrm{H},-0.1682295917,1.20$ $5720672,-3.129689072 \backslash \mathrm{H}, 2.7021884098,-1.1904102236,-2.3641930663 \backslash \mathrm{H}, 2.17$ $3627284,-1.489670749,-0.6980809696 \backslash \mathrm{H}, 1.2400327531,-2.154839226,-2.0621$ $80545 \backslash \backslash$ Version=DEC-AXP-OSF / 1-G98RevA. $11.3 \backslash \mathrm{HF}=-1068.1612165 \backslash \mathrm{RMSD}=4.100 \mathrm{e}$ $-09 \backslash \mathrm{RMSF}=2.255 \mathrm{e}-06 \backslash \mathrm{Dipole}=-0.1299334,-0.1819352,-0.4116629 \backslash \mathrm{PG}=\mathrm{C} 01] \mathrm{X}(\mathrm{C}$ $5 \mathrm{H} 1001 \mathrm{~S} 2$ ) ] \\@

\section{$\mathrm{CH}_{3} \mathrm{SC} \cdot\left(\mathrm{OC}\left(\mathrm{CH}_{3}\right)_{3}\right) \mathrm{SCH}_{3}$}

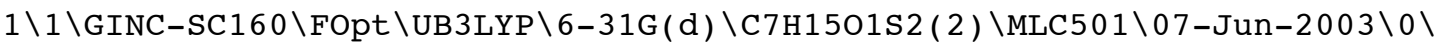
\# B3LYP/6-31G* OPT= (MAXCYC $=100$, TIGHT $)$ FREQ MAXDISK=134217728 \\rad_z=

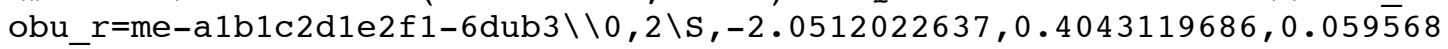
$458 \backslash \mathrm{C},-0.2713212906,0.3951909451,0.0514592196 \backslash \mathrm{S}, 0.6989830765,1.8799943$ $449,0.0390201419 \backslash \mathrm{C}, 1.0396518435,2.0953201548,-1.762913683 \backslash \mathrm{C},-2.4463174$ $333,1.8516800748,1.0991214788 \backslash 0,0.2065666959,-0.6894446763,-0.62190883$ $97 \backslash \mathrm{C}, 0.8563163854,-1.7948532634,0.1134355883 \backslash \mathrm{H},-2.0689489292,1.7179491$ $3,2.1161526517 \backslash \mathrm{H},-2.0302146546,2.7648940145,0.6689788998 \backslash \mathrm{H},-3.53725161$ $91,1.9189968361,1.123693429 \backslash \mathrm{H}, 1.4267109218,1.1607332199,-2.1728311658 \backslash$ $\mathrm{H}, 1.7958175349,2.8799245947,-1.8517850001 \backslash \mathrm{H}, 0.1336304584,2.3929850132$, $-2.2964197635 \backslash \mathrm{C}, 1.151801511,-2.8085123953,-0.9922402886 \backslash \mathrm{C},-0.109591107$ $9,-2.3719666749,1.1504934401 \backslash \mathrm{C}, 2.1474749123,-1.2979283953,0.7692354866$ $\backslash \mathrm{H}, 1.6424448654,-3.6945195462,-0.5753226558 \backslash \mathrm{H}, 0.2244730777,-3.12141730$ $42,-1.4823999081 \backslash \mathrm{H}, 1.8109579389,-2.3705732993,-1.7488246031 \backslash \mathrm{H}, 0.354486$ $7996,-3.2257665395,1.6571836934 \backslash \mathrm{H},-0.3666732949,-1.6247122904,1.907308$ $7858 \backslash \mathrm{H},-1.035104744,-2.7120871675,0.6756677705 \backslash \mathrm{H}, 2.6712805681,-2.13659$ $77065,1.242495543 \backslash \mathrm{H}, 2.8134906867,-0.8495325418,0.0254194289 \backslash \mathrm{H}, 1.939784$ $8958,-0.5472026932,1.5369885625 \backslash \backslash$ Version=DEC-AXP-OSF / 1-G03RevB . 03 \Stat $\mathrm{e}=2-\mathrm{A} \backslash \mathrm{HF}=-1147.3279774 \backslash \mathrm{S} 2=0.754752 \backslash \mathrm{S} 2-1=0 . \backslash \mathrm{S} 2 \mathrm{~A}=0.750017 \backslash \mathrm{RMSD}=9.563 e-09$ $\backslash \mathrm{RMSF}=1.806 \mathrm{e}-06 \backslash \mathrm{Dipole}=0.1685338,-0.0656418,0.098614 \backslash \mathrm{PG}=\mathrm{C} 01 \quad[\mathrm{X}(\mathrm{C} 7 \mathrm{H} 1501$ $\mathrm{S} 2) \mathrm{J} \backslash \backslash @$ 


\section{$\mathrm{S}=\mathrm{C}\left(\mathrm{OC}\left(\mathrm{CH}_{3}\right)_{3}\right) \mathrm{SCH}_{3}$}

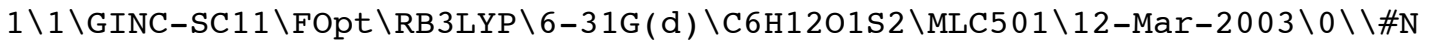
B3LYP $/ 6-31 \mathrm{G}(\mathrm{D})$ OPT $=(\mathrm{TIGHT}, \mathrm{MAXCYC}=100) \quad \mathrm{FREQ}$ MAXDISK=39321600 \} \backslash \mathrm { RAFT } ( \mathrm { Z } = \mathrm { OB } $\mathrm{u} R=\mathrm{CH} 3$ ) lowest $\mathrm{a} 1 \mathrm{~b} 1$ in $\mathrm{Cs}$ symm $\backslash \backslash 0,1 \backslash \mathrm{C},-2.8094762742,-1.973078311,0 . \backslash \mathrm{S}$ $,-0.9909272249,-2.0266499924,0 . \backslash \mathrm{C},-0.5463827548,-0.2981117822,0 . \backslash \mathrm{S},-1$. $6190708437,0.9612038617,0 . \backslash 0,0.7824311486,-0.289547251,0 . \backslash \mathrm{C}, 1.68430479$ $23,0.8956108337,0 . \backslash \mathrm{H},-3.1313924883,-3.0185738969,0 . \backslash \mathrm{H},-3.1833163326,-1$ $.4669879944,0.8918110025 \backslash \mathrm{H},-3.1833163326,-1.4669879944,-0.8918110025 \backslash \mathrm{C}$ $, 1.4810774164,1.711623448,1.2779179042 \backslash \mathrm{C}, 1.4810774164,1.711623448,-1.2$ $779179042 \backslash \mathrm{H}, 0.506186353,2.2002055556,1.2986143138 \backslash \mathrm{H}, 0.506186353,2.2002$ $055556,-1.2986143138 \backslash \mathrm{H}, 1.5747551593,1.0694331401,2.1599032388 \backslash \mathrm{H}, 1.5747$ $551593,1.0694331401,-2.1599032388 \backslash \mathrm{H}, 2.2586679702,2.4818914203,1.333659$ $7933 \backslash \mathrm{H}, 2.2586679702,2.4818914203,-1.3336597933 \backslash \mathrm{C}, 3.0603127374,0.224269$ $7459,0 . \backslash \mathrm{H}, 3.8449479523,0.9877323386,0 . \backslash \mathrm{H}, 3.1844490722,-0.4031754401,0$. $8879541515 \backslash \mathrm{H}, 3.1844490722,-0.4031754401,-0.8879541515 \backslash \backslash$ Version=DEC-AXP -OSF $/ 1-G 98 R e v A .11 .3 \backslash$ State $^{2}=1-\mathrm{A}^{\prime} \backslash \mathrm{HF}=-1107.4708739 \backslash \mathrm{RMSD}=9.707 \mathrm{e}-09 \backslash \mathrm{RMSF}=1$. $552 \mathrm{e}-06 \backslash \mathrm{Dipole}=0.5651463,-0.0509505,0 . \backslash \mathrm{PG}=\mathrm{CS} \quad[\mathrm{SG}(\mathrm{C} 4 \mathrm{H} 2 \mathrm{O} 1 \mathrm{~S} 2), \mathrm{X}(\mathrm{C} 2 \mathrm{H} 10)] \backslash \backslash$ a 
Table S4. Geometries of the RAFT-adduct Radicals $\left(\mathrm{CH}_{3} \mathrm{SC} \bullet(\mathrm{Z}) \mathrm{S}-\mathrm{R}\right)$, Used in Calculating the Beta-Scission Enthalpies in Table 4

\author{
$\mathrm{CH}_{3} \mathrm{SC} \cdot(\mathrm{CN}) \mathrm{SCH}_{2} \mathrm{OCOCH}_{3}$

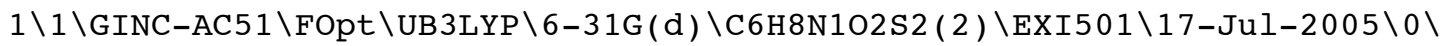 \\ $\backslash \# P$ GFINPUT B3LYP/6-31G* SCF $=(\mathrm{QC}, \mathrm{MAXCYC}=100)$ OPT $=(\mathrm{MAXCYC}=100)$ INT(GRID \\ $=U L T R A F I N E)$ MAXDISK=134217728\\RRAD ( $Z=C N R=V A) \backslash \backslash 0,2 \backslash C,-2.5401378346,-0$ \\ $.6022569174,-2.9698083642 \backslash \mathrm{S},-2.2252509381,-0.1982666185,-1.2129335199 \backslash$ \\ $C,-0.5896289946,-0.7811147915,-0.9805007538 \backslash \mathrm{S},-0.1409843035,-1.1763051$ \\ $158,0.6787728097 \backslash C, 1.5326942578,-0.4223728979,0.8070278713 \backslash 0,1.5044139$ \\ $054,0.9859367712,0.9922439415 \backslash \mathrm{C}, 1.3449086723,1.418135467,2.2818984126 \backslash$ \\ $0,1.2531882286,0.6675216028,3.2238963667 \backslash \mathrm{C}, 1.3004776639,2.9233350575,2$ \\ $.3301809057 \backslash \mathrm{H}, 1.264589069,3.2516897107,3.3692362386 \backslash \mathrm{H}, 0.4128281584,3.2$ \\ $821934392,1.7980468381 \backslash \mathrm{H}, 2.1759917145,3.3469412102,1.8285394564 \backslash \mathrm{H}, 2.08$ \\ $24935712,-0.6000746946,-0.1170587342 \backslash \mathrm{H}, 2.0065915497,-0.9168853285,1.65$ \\ $63925159 \backslash \mathrm{C}, 0.3101215164,-0.9231926687,-2.0344768397 \backslash \mathrm{N}, 1.0820732801,-1$. \\ $03683887,-2.9127296099 \backslash \mathrm{H},-1.8086751165,-0.1167042852,-3.6191698188 \backslash \mathrm{H},-$ \\ $2.5219505321,-1.6832302782,-3.1245637011 \backslash \mathrm{H},-3.5380462684,-0.2157764204$ \\ ,$-3.1907940199 \backslash \backslash$ Version=IA64L-G03RevC.02 \State=2-A \HF=-1234.9849486\S2 \\ $=0.760069 \backslash S 2-1=0 . \backslash S 2 A=0.750065 \backslash R M S D=0.000 e+00 \backslash R M S F=8.297 e-07 \backslash D i p o l e=-0$ \\ $.6067495,0.6846731,-0.1850487 \backslash \mathrm{PG}=\mathrm{C} 01 \quad[\mathrm{X}(\mathrm{C} 6 \mathrm{H} 8 \mathrm{~N} 102 \mathrm{~S} 2)] \backslash \backslash \mathrm{a}$
}

\title{
$\mathrm{CH}_{3} \mathrm{SC} \cdot(\mathrm{F}) \mathrm{SCH}_{2} \mathrm{OCOCH}_{3}$
}

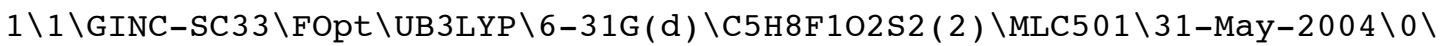
$\backslash \#$ B3LYP $/ 6-31 \mathrm{G} *$ OPT $=($ MAXCYC $=300)$ FREQ GEOM=CHECK GUESS=READ MAXDISK=40

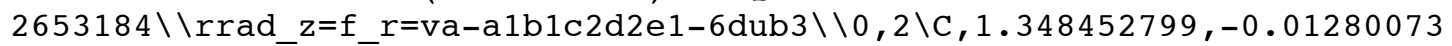
$78,0.32334631 \overline{2} 7 \backslash \mathrm{F}, 1.6683273236,-0.8665081215,1.3387796557 \backslash \mathrm{S}, 2.58689574$ $65,1.117692045,-0.1432312448 \backslash \mathrm{S}, 0.1585089702,-0.6139234859,-0.82131261 \backslash$ $\mathrm{C}, 4.0668370788,0.0275225816,-0.3208345924 \backslash \mathrm{C},-1.1533597783,-1.160199762$ $1,0.3463735784 \backslash \mathrm{H},-1.7210213906,-1.9322685529,-0.1739449799 \backslash \mathrm{H},-0.676419$ $0801,-1.5469082514,1.2449018386 \backslash 0,-2.011595768,-0.106284409,0.77116814$ $52 \backslash \mathrm{C},-3.0321720436,0.2100790435,-0.0807637613 \backslash 0,-3.2320378877,-0.37315$ $94217,-1.1204010499 \backslash \mathrm{C},-3.8336916104,1.3660113838,0.4606904532 \backslash \mathrm{H}, 4.9297$ $406674,0.6909462639,-0.4181150232 \backslash \mathrm{H}, 3.9812688647,-0.6004121882,-1.2105$ $759647 \backslash \mathrm{H}, 4.1793907233,-0.5890534484,0.5723014932 \backslash \mathrm{H},-3.2166621676,2.271$ $2946324,0.4587007794 \backslash \mathrm{H},-4.7133038827,1.5215792824,-0.1645976094 \backslash \mathrm{H},-4.1$ $317445422,1.1749740023,1.4960055357 \backslash \backslash$ Version=DEC-AXP-OSF / 1-G03RevB.03\ State $=2-A \backslash H F=-1241.9601291 \backslash S 2=0.755369 \backslash S 2-1=0 . \backslash S 2 A=0.750021 \backslash R M S D=3.210$ e-09 $\backslash$ RMSF $=3.049 e-06 \backslash D i p o l e=0.292382,-0.0780562,0.5610242 \backslash \mathrm{PG}=\mathrm{C} 01] \mathrm{X}(\mathrm{C} 5 \mathrm{H}$ $8 \mathrm{~F} 102 \mathrm{~S} 2) \mathrm{]} \backslash \backslash \mathrm{Q}$

\section{$\mathrm{CH}_{3} \mathrm{SC} \cdot\left(\mathrm{OCH}_{3}\right) \mathrm{SCH}_{2} \mathrm{OCOCH}_{3}$}

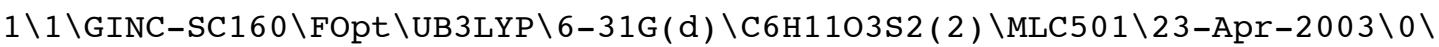
$\backslash \# \mathrm{P}$ B3LYP $/ 6-31 \mathrm{G} * \mathrm{SCF}=(\mathrm{QC}, \mathrm{MAXCYC}=100) \quad \mathrm{OPT}=(\mathrm{MAXCYC}=100) \quad \mathrm{FREQ}$ MAXDISK $=131$ $072000 \backslash \backslash \mathrm{RAFT}(\mathrm{Z}=\mathrm{OMe} \mathrm{R}=\mathrm{VA}) \mathrm{a} \mathrm{b} 1 \mathrm{c} 1 \mathrm{~d} 3 \mathrm{e} 2 \mathrm{f} 1 / / \mathrm{B} 3 \backslash \backslash 0,2 \backslash \mathrm{S}, 0.3322714222,-1.33810$ $11735,-0.9063561478 \backslash \mathrm{C}, 0.5694748971,-0.5250061298,0.6470647641 \backslash \mathrm{S}, 2.2229$ $45348,-0.384145835,1.2467459401 \backslash \mathrm{C}, 2.3646929606,-1.8402281312,2.3780173$ $258 \backslash \mathrm{C},-1.3096630931,-0.7061140865,-1.4131144165 \backslash 0,-0.4770788066,-0.633$ $9063631,1.515866466 \backslash \mathrm{C},-0.6921128753,0.4861332319,2.3945790952 \backslash 0,-1.229$ $3506832,0.4793060121,-2.2145907541 \backslash \mathrm{C},-1.4569891118,1.6707268399,-1.600$ $2930658 \backslash 0,-1.7749735518,1.7864051526,-0.4377061153 \backslash \mathrm{C},-1.2583630012,2.8$ $07316002,-2.5722486417 \backslash \mathrm{H},-1.7511406959,-1.470831824,-2.0528684105 \backslash \mathrm{H},-1$ $.9148450646,-0.5303100028,-0.5244913106 \backslash \mathrm{H}, 1.5016140583,-1.880452333,3$. $0454963331 \backslash \mathrm{H}, 3.2748778366,-1.6952499913,2.9659377291 \backslash \mathrm{H}, 2.4393739835,-2$ $.7685512536,1.8069091042 \backslash \mathrm{H},-1.8414714853,2.638407595,-3.4826197805 \backslash \mathrm{H},-$ 
$0.2036999806,2.8589667581,-2.8638987689 \backslash \mathrm{H},-1.5554863394,3.7448716146,-$ $2.1013751664 \backslash \mathrm{H},-1.5256749738,0.1957480346,3.0369865225 \backslash \mathrm{H}, 0.1974766108$, $0.681808446,3.001826327 \backslash \mathrm{H},-0.9555065988,1.3701403213,1.809273606 \backslash \backslash$ Vers ion=DEC-AXP-OSF $/ 1-G 98 R e v A .11 .3 \backslash \mathrm{HF}=-1257.2539065 \backslash \mathrm{S} 2=0.754973 \backslash \mathrm{S} 2-1=0 . \backslash \mathrm{S} 2$ $\mathrm{A}=0.750018 \backslash \mathrm{RMSD}=0.000 \mathrm{e}+00 \backslash \mathrm{RMSF}=9.849 \mathrm{e}-06 \backslash \mathrm{Dipole}=-0.3783478,0.0568044,0$ $.05398 \backslash \mathrm{PG}=\mathrm{C} 01 \quad \mathrm{X}(\mathrm{C} 6 \mathrm{H} 1103 \mathrm{~S} 2)] \backslash \backslash @$

\section{$\mathrm{CH}_{3} \mathrm{SC} \cdot(\mathrm{CN}) \mathrm{SCH}_{2} \mathrm{Ph}$}

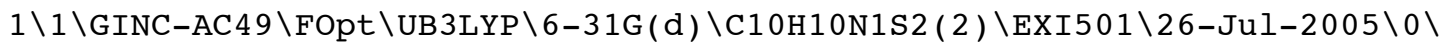
$\backslash \# \mathrm{P}$ GFINPUT B3LYP/6-31G* SCF $=(\mathrm{QC}, \mathrm{MAXCYC}=100) \quad \mathrm{OPT}=(\mathrm{MAXCYC}=100)$ INT $(\mathrm{GRID}$ =ULTRAFINE) MAXDISK=134217728 IOP $(1 / 8=10) \backslash \backslash R R A D(Z=C N R=B Z) \backslash \backslash 0,2 \backslash C,-1.9$ $266885076,1.611394121,-2.7553783841 \backslash \mathrm{C},-1.9267280366,1.6113654132,-1.36$ $21043006 \backslash \mathrm{C},-0.7202427712,1.6113570021,-0.6475144955 \backslash \mathrm{C}, 0.4877889568,1.6$ $108112332,-1.3571530673 \backslash \mathrm{C}, 0.4881139113,1.6099474873,-2.753342189 \backslash \mathrm{C},-0$. $7173070983,1.6099316222,-3.4554942396 \backslash \mathrm{C},-0.7211463448,1.614016551,0.85$ $71467634 \backslash \mathrm{S},-1.0546823114,-0.0468780865,1.6395337246 \backslash \mathrm{C}, 0.4179742724,-0$. $9392852264,1.3049438653 \backslash \mathrm{S}, 0.214579504,-2.6207329159,0.846351902 \backslash \mathrm{C}, 1.78$ $18325871,-3.3734325664,1.4193318476 \backslash \mathrm{C}, 1.6732449897,-0.3391468704,1.384$ $05191 \backslash \mathrm{N}, 2.7253907353,0.1808602092,1.4411014699 \backslash \mathrm{H},-1.5305591838,2.22996$ $11687,1.2621342523 \backslash \mathrm{H}, 0.2217391736,1.9863707061,1.2659127849 \backslash \mathrm{H}, 1.429858$ $3296,1.6129777517,-0.8152119255 \backslash \mathrm{H}, 1.432751554,1.6120837632,-3.29046343$ $56 \backslash \mathrm{H},-0.716576827,1.6114509709,-4.5421557315 \backslash \mathrm{H},-2.8696514683,1.6154178$ $496,-3.2955491517 \backslash \mathrm{H},-2.8707174631,1.6093561817,-0.8214515904 \backslash \mathrm{H}, 2.64167$ $10211,-2.88391238,0.9569274036 \backslash \mathrm{H}, 1.8592378538,-3.3207301545,2.50755366$ $24 \backslash \mathrm{H}, 1.7451050284,-4.4189738852,1.1034971542 \backslash \backslash$ Version=IA64L-G03RevC.02 $\backslash$ State $=2-\mathrm{A} \backslash \mathrm{HF}=-1238.1630334 \backslash \mathrm{S} 2=0.759535 \backslash \mathrm{S} 2-1=0 . \backslash \mathrm{S} 2 \mathrm{~A}=0.75006 \backslash \mathrm{RMSD}=0.000$ $\mathrm{e}+00 \backslash \mathrm{RMSF}=1.618 \mathrm{e}-06 \backslash \mathrm{Dipole}=-0.5456296,-0.3722925,-0.3443287 \backslash \mathrm{PG}=\mathrm{C} 01] \mathrm{X}($ C10H10N1S2) ] \\@

\section{$\mathrm{CH}_{3} \mathrm{SC} \cdot(\mathrm{F}) \mathrm{SCH}_{2} \mathrm{Ph}$}

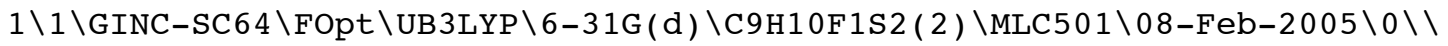
\# B3LYP/6-31G* OPT MAXDISK=268435456\\rrad $z=f \quad r=b z-a 1 b 1 c 3-6 d u b 3 . c o m \backslash \backslash$ $0,2 \backslash \mathrm{C},-1.5136034022,-0.031467307,0.1113687 \overline{6} 82 \backslash \overline{\mathrm{S}},-2.6458718156,-0.88337$ $20036,-0.8949930098 \backslash \mathrm{S},-0.8608398296,1.5297718896,-0.3149842014 \backslash \mathrm{F},-1.57$ $56438353,-0.3025559488,1.4522309625 \backslash C,-4.2565322424,-0.5282128085,-0.0$ $622993251 \backslash \mathrm{C}, 0.6697388339,1.563834948,0.7552322365 \backslash \mathrm{H},-4.9964630942,-1.1$ $88536116,-0.5216817746 \backslash \mathrm{H},-4.5484601548,0.5140176525,-0.2103222061 \backslash \mathrm{H},-4$ $.1774474444,-0.754187307,1.0024080191 \backslash \mathrm{H}, 0.3351539866,1.4129209182,1.78$ $38332562 \backslash \mathrm{H}, 1.0204312311,2.5953599695,0.6577128882 \backslash \mathrm{C}, 1.7306065625,0.574$ $7654699,0.3584730318 \backslash \mathrm{C}, 1.7007923138,-0.7350572128,0.857615279 \backslash \mathrm{C}, 2.7601$ $437566,0.941139805,-0.518259018 \backslash \mathrm{H}, 0.9027907579,-1.0308562758,1.5328561$ $842 \backslash \mathrm{H}, 2.7924200265,1.9540557845,-0.9137516677 \backslash \mathrm{C}, 2.6810218592,-1.656033$ $0296,0.4877805126 \backslash \mathrm{C}, 3.7416311043,0.0217293085,-0.8870469162 \backslash \mathrm{H}, 2.646001$ $5521,-2.6676166488,0.8834747583 \backslash \mathrm{H}, 4.536188496,0.3227299061,-1.56468543$ $55 \backslash \mathrm{C}, 3.7042866933,-1.2805983612,-0.3845644261 \backslash \mathrm{H}, 4.4690526097,-1.997887$ $3957,-0.6700881613 \backslash \backslash$ Version=DEC-AXP-OSF $/ 1-G 03$ RevB .03 $\backslash$ State $=2-A \backslash H F=-124$ $5.1379942 \backslash \mathrm{S} 2=0.75568 \backslash \mathrm{S} 2-1=0 . \backslash \mathrm{S} 2 \mathrm{~A}=0.750023 \backslash \mathrm{RMSD}=8.940 \mathrm{e}-09 \backslash \mathrm{RMSF}=4.967 \mathrm{e}-0$ $6 \backslash$ Dipole $=-0.0163509,0.1894396,0.323868 \backslash \mathrm{PG}=\mathrm{C} 01 \quad[\mathrm{X}(\mathrm{C} 9 \mathrm{H} 10 \mathrm{~F} 1 \mathrm{~S} 2)] \backslash \backslash @$

\section{$\mathrm{CH}_{3} \mathrm{SC} \cdot\left(\mathrm{OCH}_{3}\right) \mathrm{SCH}_{2} \mathrm{Ph}$}

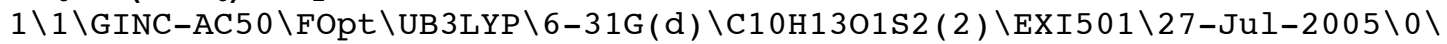
$\backslash \# \mathrm{P}$ B3LYP $/ 6-31 \mathrm{G} * \mathrm{SCF}=(\mathrm{QC}, \mathrm{MAXCYC}=100) \quad \mathrm{OPT}=(\mathrm{MAXCYC}=100)$ INT $(\mathrm{GRID}=\mathrm{ULTRAFI}$ $\mathrm{NE}) \mathrm{MAXDISK}=134217728 \backslash \backslash \mathrm{RAFT}(\mathrm{Z}=\mathrm{OMe} \mathrm{R}=\mathrm{BZ}) \backslash \backslash 0,2 \backslash \mathrm{C}, 0.1347339529,-1.3500862$ $936,-2.5789778746 \backslash \mathrm{C}, 0.1059492423,-1.431665444,-1.1781630713 \backslash \mathrm{C}, 1.323592$ $23,-1.5012433289,-0.4861725281 \backslash \mathrm{C}, 2.5371590906,-1.4911278088,-1.1746769$ $455 \backslash \mathrm{C}, 2.5530246748,-1.4107904153,-2.568211168 \backslash \mathrm{C}, 1.3463067151,-1.339892$ $938,-3.268902547 \backslash C,-1.1957678008,-1.4326739321,-0.4339264213 \backslash \mathrm{S},-1.9462$ 
$548192,0.2862649554,-0.1290687847 \backslash \mathrm{C},-0.9036645277,1.0590459003,1.04169$ $08386 \backslash 0,0.329734305,1.5637350006,0.7371939618 \backslash \mathrm{C}, 0.4658777173,2.2639864$ $111,-0.5035289979 \backslash \mathrm{S},-1.0873723568,0.5856682854,2.7330985905 \backslash \mathrm{C},-0.02164$ $73966,1.8065465661,3.5863311187 \backslash \mathrm{H}, 1.3171056373,-1.5612136773,0.5992733$ $003 \backslash \mathrm{H}, 3.4709094427,-1.5493512425,-0.621403345 \backslash \mathrm{H}, 3.4977343392,-1.406894$ $6085,-3.1053216124 \backslash \mathrm{H}, 1.3488869518,-1.2822239656,-4.3543342064 \backslash \mathrm{H},-0.801$ $6940065,-1.2956975099,-3.129915293 \backslash \mathrm{H},-1.986966785,-1.9354289959,-0.999$ $6254284 \backslash \mathrm{H},-1.1087972016,-1.9159458061,0.5419344887 \backslash \mathrm{H},-0.2792550133,3.0$ $639682594,-0.5808999679 \backslash \mathrm{H}, 1.4714812863,2.6885528579,-0.4889295621 \backslash \mathrm{H}, 0$. $3605798464,1.5831557907,-1.3528827056 \backslash \mathrm{H}, 1.0189072659,1.6975965642,3.27$ $8622495 \backslash \mathrm{H},-0.1160802082,1.5964977414,4.6548902511 \backslash \mathrm{H},-0.366034566,2.823$ $5804325,3.3837885773 \backslash \backslash$ Version=IA64L-G03RevC .02 $\backslash$ State $=2-A \backslash H F=-1260.4304$ $622 \backslash \mathrm{S} 2=0.755277 \backslash \mathrm{S} 2-1=0 . \backslash \mathrm{S} 2 \mathrm{~A}=0.750021 \backslash \mathrm{RMSD}=0.000 \mathrm{e}+00 \backslash \mathrm{RMSF}=1.842 \mathrm{e}-06 \backslash \mathrm{Dip}$ $\mathrm{ole}=0.8362651,0.2519536,-0.2291334 \backslash \mathrm{PG}=\mathrm{C} 01 \quad[\mathrm{X}(\mathrm{C} 10 \mathrm{H} 1301 \mathrm{~S} 2)] \backslash \backslash @$

\section{$\mathrm{CH}_{3} \mathrm{SC} \cdot(\mathrm{CN}) \mathrm{SC}\left(\mathrm{CH}_{3}\right)_{2} \mathrm{CN}$}

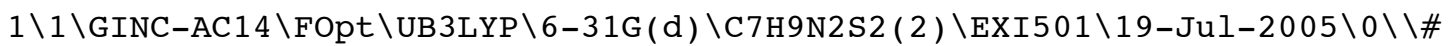
$P$ GFINPUT B3LYP $/ 6-31 \mathrm{G} * \mathrm{SCF}=(\mathrm{QC}, \mathrm{MAXCYC}=100) \quad \mathrm{OPT}=(\mathrm{MAXCYC}=100)$ INT $(\mathrm{GRID}=\mathrm{U}$

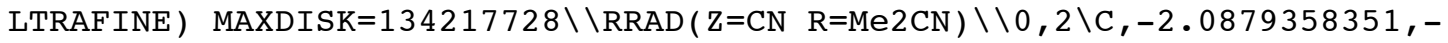
$0.8923208483,-2.6940394003 \backslash S,-1.8554048897,-0.9360446123,-0.8813209579$ $\backslash \mathrm{C},-0.1267771681,-0.7868387711,-0.7016641801 \backslash \mathrm{S}, 0.5076391422,-0.9602756$ $355,0.9318518357 \backslash \mathrm{C}, 0.9286875247,0.8106473508,1.4803716234 \backslash \mathrm{C},-0.2737140$ $518,1.6381115004,1.3439494321 \backslash \mathrm{C}, 1.3108868957,0.6654135645,2.966423497 \backslash$ $\mathrm{C}, 2.0845534849,1.3972291074,0.6541322113 \backslash \mathrm{C}, 0.7150989234,-0.6014731356$, $-1.8014023626 \backslash \mathrm{N},-1.2174993678,2.3060526905,1.2290453489 \backslash \mathrm{N}, 1.4118618011$ $,-0.4389678088,-2.7321228194 \backslash \mathrm{H},-1.7438560951,0.0592385975,-3.104721399$ $6 \backslash \mathrm{H},-3.1629721753,-0.9962294292,-2.8589237967 \backslash \mathrm{H},-1.5598598621,-1.71969$ $51003,-3.173117776 \backslash \mathrm{H}, 1.8311356337,1.4694939657,-0.4057072487 \backslash \mathrm{H}, 2.96909$ $64727,0.7625709598,0.7593796916 \backslash \mathrm{H}, 2.3243810707,2.4026438981,1.01920592$ $64 \backslash \mathrm{H}, 2.1740494149,-0.0009442596,3.0632935463 \backslash \mathrm{H}, 1.5841662801,1.64556416$ $41,3.3703581718 \backslash \mathrm{H}, 0.4827755451,0.2642743872,3.5566562078 \backslash \backslash$ Version=IA64 $\mathrm{L}-\mathrm{G} 03$ RevC.02 $\backslash \mathrm{State}=2-\mathrm{A} \backslash \mathrm{HF}=-1177.9724758 \backslash \mathrm{S} 2=0.760291 \backslash \mathrm{S} 2-1=0 . \backslash \mathrm{S} 2 \mathrm{~A}=0.7500$ $7 \backslash \mathrm{RMSD}=0.000 \mathrm{e}+00 \backslash \mathrm{RMSF}=4.356 \mathrm{e}-06 \backslash \mathrm{Dipole}=0.0641694,-0.4299218,0.4071566 \backslash$ $\mathrm{PG}=\mathrm{C} 01 \quad \mathrm{X}(\mathrm{C} 7 \mathrm{H} 9 \mathrm{~N} 2 \mathrm{~S} 2)] \backslash \backslash @$

\section{$\mathrm{CH}_{3} \mathrm{SC} \cdot(\mathrm{F}) \mathrm{SC}\left(\mathrm{CH}_{3}\right)_{2} \mathrm{CN}$}

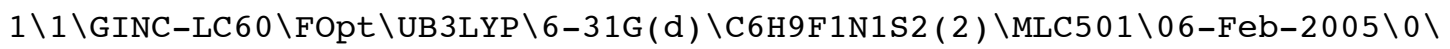

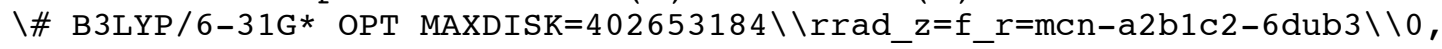
$2 \backslash \mathrm{C},-0.9134774371,-1.0737174345,0.184140730 \overline{6} \backslash \mathrm{S},-1.2408597808,-0.877263$ $7323,1.8928036739 \backslash \mathrm{S}, 0.5423284116,-0.9458858803,-0.7016465697 \backslash \mathrm{F},-2.0881$ $209535,-1.0869843621,-0.5029857515 \backslash \mathrm{C}, 0.4306616681,-0.8570862568,2.6247$ $566158 \backslash \mathrm{C}, 0.71085958,0.919547555,-1.2495062891 \backslash \mathrm{H}, 0.9704564866,0.0445114$ $714,2.3305436075 \backslash \mathrm{H}, 0.2712772858,-0.853336266,3.7060664279 \backslash \mathrm{H}, 0.98764918$ $72,-1.7521479637,2.3416410148 \backslash \mathrm{C}, 0.9825412872,1.7033429155,-0.046829466$ $3 \backslash \mathrm{C},-0.5766770692,1.3845042271,-1.9406388817 \backslash \mathrm{C}, 1.9243923461,0.93111197$ $18,-2.1922556738 \backslash \mathrm{H},-1.4399446953,1.3081808216,-1.2759042651 \backslash \mathrm{H},-0.76262$ $34676,0.7648625972,-2.8226351821 \backslash \mathrm{H},-0.474081186,2.4292271047,-2.257692$ $4877 \backslash \mathrm{H}, 2.8282121456,0.5749889652,-1.6893102257 \backslash \mathrm{H}, 1.7256905182,0.291798$ $9149,-3.0579709662 \backslash \mathrm{H}, 2.108740063,1.9508703524,-2.5478456103 \backslash \mathrm{N}, 1.200630$ $2716,2.3068684563,0.9233519386 \backslash \backslash$ Version=x86-Linux-G03RevB.03 $\backslash$ State $=2-A$ $\backslash \mathrm{HF}=-1184.9514909 \backslash \mathrm{S} 2=0.756645 \backslash \mathrm{S} 2-1=0 . \backslash \mathrm{S} 2 \mathrm{~A}=0.75003 \backslash \mathrm{RMSD}=2.657 \mathrm{e}-09 \backslash \mathrm{RMSF}=$ $3.246 e-06 \backslash \mathrm{Dipole}=0.4192358,-0.2267913,-0.6436795 \backslash \mathrm{PG}=\mathrm{C} 01$ [X(C6H9F1N1S2) ]$\backslash \backslash @$

\section{$\mathrm{CH}_{3} \mathrm{SC} \cdot\left(\mathrm{OCH}_{3}\right) \mathrm{SC}\left(\mathrm{CH}_{3}\right)_{2} \mathrm{CN}$}

$1 \backslash 1 \backslash G I N C-A C 52 \backslash F O p t \backslash U B 3 L Y P \backslash 6-31 G(d) \backslash C 7 H 12 N 101 S 2$ ( 2) \EXI501\25-Jul-2005 0 $\backslash \backslash \# \mathrm{P}$ B3LYP $/ 6-31 \mathrm{G} * \mathrm{SCF}=(\mathrm{QC}, \mathrm{MAXCYC}=100)$ OPT $=(\mathrm{MAXCYC}=100) \quad$ INT $(\mathrm{GRID}=\mathrm{ULTRAF}$ 
INE ) MAXDISK=134217728\\RAFT ( $\mathrm{Z}=\mathrm{OMe} \mathrm{R}=\mathrm{Me} 2 \mathrm{CN}) \backslash \backslash 0,2 \backslash \mathrm{C},-3.0505312151,1.229$ $1517167,-1.6576419406 \backslash \mathrm{S},-2.3478261878,0.2637960664,-0.2691242584 \backslash \mathrm{C},-0$. $7044067616,-0.0463195491,-0.8299229419 \backslash 0,-0.2103857733,0.9070545502,-1$ $.6618536739 \backslash \mathrm{C}, 0.9789932357,0.6183062477,-2.4115416155 \backslash \mathrm{S}, 0.2267213045,-$ $1.2838322423,-0.0685700116 \backslash \mathrm{C}, 1.1507960875,-0.5350443876,1.4965496701 \backslash \mathrm{C}$ , $2.1656255906,0.3946259296,1.0098239203 \backslash \mathrm{C}, 1.8055710333,-1.7626211926,2$ $.1490127199 \backslash \mathrm{C}, 0.1573807977,0.1661788613,2.4275981908 \backslash \mathrm{H},-3.0133441234,0$ $.6521863003,-2.5845918078 \backslash \mathrm{H},-4.0923672527,1.4227880785,-1.3896003969 \backslash \mathrm{H}$ $,-2.5193318388,2.1731503165,-1.7830112749 \backslash \mathrm{H}, 1.8550912459,0.5936788338$, $-1.7586474978 \backslash \mathrm{H}, 1.0709086685,1.4393538099,-3.1245009077 \backslash \mathrm{H}, 0.8797595332$ $,-0.3337963138,-2.9431711944 \backslash \mathrm{N}, 2.9697389284,1.1309833308,0.6039510162 \backslash$ $\mathrm{H}, 2.4886441112,-2.2672372994,1.459461308 \backslash \mathrm{H}, 1.0318099055,-2.4711291519$, $2.4607582158 \backslash \mathrm{H}, 2.3746931348,-1.4572128293,3.0342429987 \backslash \mathrm{H}, 0.6798440591$, $0.5521846014,3.3116833375 \backslash \mathrm{H},-0.6060350171,-0.5459708543,2.7546758245 \backslash \mathrm{H}$ $,-0.337653213,1.0035978496,1.9297139738 \backslash \backslash$ Version=IA64L-G03RevC . 02 \Stat $\mathrm{e}=2-\mathrm{A} \backslash \mathrm{HF}=-1200.2455939 \backslash \mathrm{S} 2=0.75636 \backslash \mathrm{S} 2-1=0 . \backslash \mathrm{S} 2 \mathrm{~A}=0.750028 \backslash \mathrm{RMSD}=0.000 \mathrm{e}+00 \backslash$ $\mathrm{RMSF}=3.701 \mathrm{e}-06 \backslash \mathrm{Dipole}=-0.7811189,-0.2699558,-0.1055335 \backslash \mathrm{PG}=\mathrm{C} 01] \mathrm{X}(\mathrm{C} 7 \mathrm{H} 12$ N101S2 ) ] \\@ 